\title{
Computationally Guided Tuning of Amino Acid Configuration Influences the Chiroptical Properties of Supramolecular Peptide- $\pi$-Peptide Nanostructures
}

Sayak Subhra Panda, ${ }^{\dagger, *}$ Kirill Shmilovich ${ }^{\mathbb{I}}$, Andrew L. Ferguson ${ }^{\mathbb{I l}}$, and John D. Tovar ${ }^{*}, \dagger,+$,

$\dagger$ Department of Chemistry, Johns Hopkins University, 3400 North Charles Street, Baltimore, Maryland 21218, United States.

* Institute of NanoBioTechnology, Johns Hopkins University, 3400 North Charles Street, Baltimore, Maryland, 21218, United States.

${ }^{\perp}$ Department of Materials Science and Engineering, Johns Hopkins University, 3400 North Charles Street, Baltimore, Maryland 21218, United States.

II Pritzker School of Molecular Engineering, University of Chicago, 5640 South Ellis Avenue, Chicago, Illinois, 60637, United States.

"Corresponding author email: tovar@jhu.edu

\section{Table of Contents}

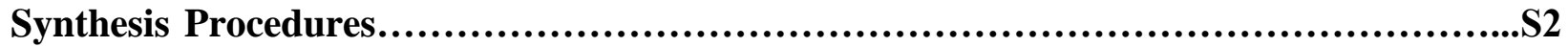

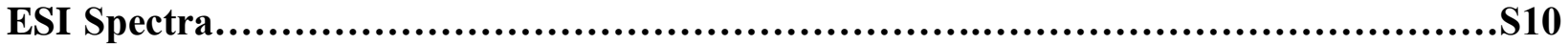

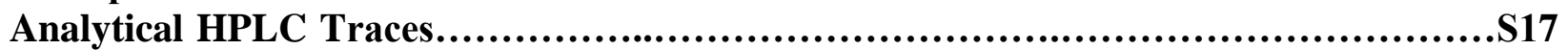

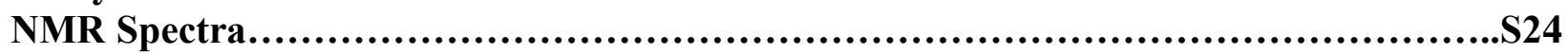

Electron Microscopy \& TEM Images.....................................................S31

DLS Spectra..............................................................................

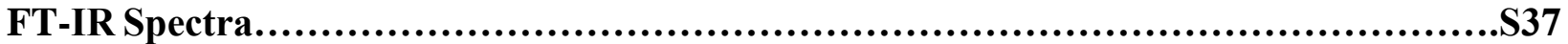

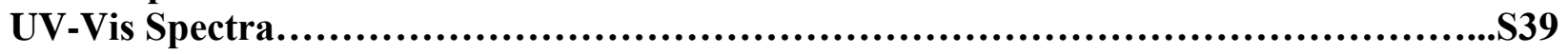

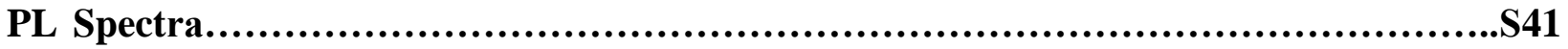

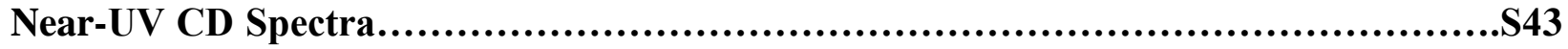

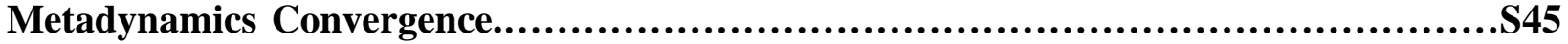

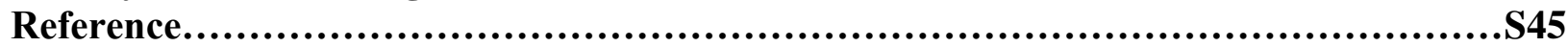




\section{Synthesis Procedures:}

OH-VEVLALG-P-T-P-GALV $V_{L}$ EV-OH: Prepared according to literature procedure and characterization matched that of literature. ${ }^{1}$

OH-VEVLAdG-P-T-P-GAdVLEV-OH: Solid supported Wang-VEV $\mathrm{L}_{\mathrm{D}} \mathrm{G}_{\mathrm{L}} \mathrm{NH}_{2}$ peptide Nacylated with 4 -iodobenzoic acid was prepared $(0.10 \mathrm{mmol})$. The resin was subjected to the standard Stille coupling procedure in the presence of 2,5-bis(trimethylstannyl)thiophene $(0.050$ mmol, $0.021 \mathrm{~g})$ and $\mathrm{Pd}\left(\mathrm{PPh}_{3}\right)_{4}(0.0040 \mathrm{mmol}, 0.0046 \mathrm{~g})$ for $21 \mathrm{~h}$. Following general cleavage and work-up, the peptide was obtained as a white powder $(0.033 \mathrm{mmol}, 0.041 \mathrm{~g}, 33 \%$ yield $)$. Following HPLC purification, $0.0089 \mathrm{mmol}, 0.011 \mathrm{~g}, 8.9 \%$ yield. ${ }^{1} \mathrm{H}$ NMR $\left(400 \mathrm{MHz}, \mathrm{D}_{2} \mathrm{O}\right) \delta: 7.87$ (d, $1 \mathrm{H}$, $J=8.72 \mathrm{~Hz}), 7.78($ br s, 6H), $7.53(\mathrm{~s}, 2 \mathrm{H}), 7.49(\mathrm{~d}, 1 \mathrm{H}, J=8.72 \mathrm{~Hz}), 4.35(\mathrm{q}, 2 \mathrm{H}, J=7.24 \mathrm{~Hz})$, $4.27(\mathrm{dd}, 2 \mathrm{H}, J=7.44 \mathrm{~Hz}), 4.10(\mathrm{~d}, 2 \mathrm{H}, J=7.44 \mathrm{~Hz}), 4.08-4.03(\mathrm{~m}, 4 \mathrm{H}), 3.97(\mathrm{~d}, 2 \mathrm{H}, J=5.96 \mathrm{~Hz})$, 2.23-2.12 (m, 4H), 2.10-1.90 (m, 6H), 1.89-1.77 (m, 2H), $1.35(\mathrm{~d}, 6 \mathrm{H}, J=7.28 \mathrm{~Hz}), 0.86(\mathrm{~d}, 6 \mathrm{H}$, $J=6.92 \mathrm{~Hz}), 0.83(\mathrm{~d}, 6 \mathrm{H}, J=2.50 \mathrm{~Hz}), 0.82(\mathrm{~d}, 6 \mathrm{H}, J=2.60 \mathrm{~Hz}), 0.79(\mathrm{~d}, 6 \mathrm{H}, J=6.82 \mathrm{~Hz}) . \mathrm{UV}-$ Vis $\left(\mathrm{H}_{2} \mathrm{O}\right) \lambda / \mathrm{nm}: 352(\mathrm{pH} 10)$. MS (ESI) m/z $654.4(\mathrm{M}+2 \mathrm{~K}-4 \mathrm{H})^{-}$(calc. 655.1), m/z 635.5 (M+K3H $)^{2-}\left(\right.$ calc. 635.7), m/z $616.7(\mathrm{M}-2 \mathrm{H})^{2-}\left(\right.$ calc. 616.7), m/z $423.4(\mathrm{M}+\mathrm{K}-4 \mathrm{H})^{3-}$ (calc. 423.5), m/z $411.0(\mathrm{M}-3 \mathrm{H})^{3-}($ calc. 410.8$), \mathrm{m} / \mathrm{z} 307.8(\mathrm{M}-4 \mathrm{H})^{4-}$ (calc. 307.9).

OH-VEVDALG-P-T-P-GALVDEV-OH: Solid supported Wang-VEV DA $_{L} \mathrm{G}_{\mathrm{N}} \mathrm{NH}_{2}$ peptide Nacylated with 4-iodobenzoic acid was prepared $(0.10 \mathrm{mmol})$. The resin was subjected to the standard Stille coupling procedure in the presence of 2,5-bis(trimethylstannyl)thiophene $(0.050$ mmol, $0.021 \mathrm{~g})$ and $\mathrm{Pd}\left(\mathrm{PPh}_{3}\right)_{4}(0.0040 \mathrm{mmol}, 0.0046 \mathrm{~g})$ for $21 \mathrm{~h}$. Following general cleavage and work-up, the peptide was obtained as a white powder ( $0.042 \mathrm{mmol}, 0.052 \mathrm{~g}, 42 \%$ yield). Following HPLC purification, $0.013 \mathrm{mmol}, 0.016 \mathrm{~g}, 13 \%$ yield. ${ }^{1} \mathrm{H}$ NMR $\left(400 \mathrm{MHz}, \mathrm{D}_{2} \mathrm{O}\right) \delta: 7.86(\mathrm{~d}, 3 \mathrm{H}, J$ $=8.44 \mathrm{~Hz}), 7.80-7.68(\mathrm{~m}, 3 \mathrm{H}),, 7.50(\mathrm{~d}, 4 \mathrm{H}, J=8.56 \mathrm{~Hz}), 4.37-4.30(\mathrm{~m}, 2 \mathrm{H}), 4.26(\mathrm{q}, 2 \mathrm{H}, J=5.04$ 
Hz), 4.11-3.97 (m, 6H), 3.95 (d, 2H, J = 5.88 Hz), 2.19-2.04 (m, 6H), 2.03-1.89 (m, 4H), 1.87$1.76(\mathrm{~m}, 2 \mathrm{H}), 1.32(\mathrm{~d}, 6 \mathrm{H}, J=7.16 \mathrm{~Hz}), 0.86(\mathrm{~d}, 6 \mathrm{H}, J=6.96 \mathrm{~Hz}), 0.82(\mathrm{~d}, 6 \mathrm{H}, J=7.00 \mathrm{~Hz}), 0.75$ (t, $12 \mathrm{H}, J=6.42 \mathrm{~Hz})$. UV-Vis $\left(\mathrm{H}_{2} \mathrm{O}\right) \lambda / \mathrm{nm}: 351(\mathrm{pH} 10)$. MS (ESI) m/z $1347.6(\mathrm{M}+3 \mathrm{~K}-4 \mathrm{H})^{-}$(calc.

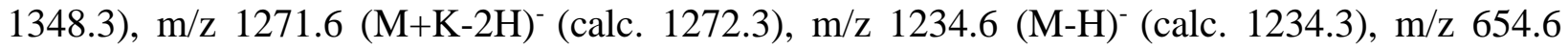
$(\mathrm{M}+2 \mathrm{~K}-4 \mathrm{H})^{2-}\left(\right.$ calc. 654.7), m/z 635.2 $(\mathrm{M}+\mathrm{K}-3 \mathrm{H})^{2-}\left(\right.$ calc. 635.7), m/z $616.9(\mathrm{M}-2 \mathrm{H})^{2-}($ calc. 616.7). OH-VEVDADG-P-T-P-GADVDEV-OH: Solid supported Wang-VEV ${ }_{D} A_{D} G-N_{2}$ peptide Nacylated with 4-iodobenzoic acid was prepared $(0.10 \mathrm{mmol})$. The resin was subjected to the standard Stille coupling procedure in the presence of 2,5-bis(trimethylstannyl)thiophene $(0.050$ mmol, $0.021 \mathrm{~g})$ and $\mathrm{Pd}\left(\mathrm{PPh}_{3}\right)_{4}(0.0040 \mathrm{mmol}, 0.0046 \mathrm{~g})$ for $21 \mathrm{~h}$. Following general cleavage and work-up, the peptide was obtained as a white powder ( $0.048 \mathrm{mmol}, 0.059 \mathrm{~g}, 48 \%$ yield $)$. Following HPLC purification, $0.015 \mathrm{mmol}, 0.019 \mathrm{~g}, 15 \%$ yield. ${ }^{1} \mathrm{H}$ NMR $\left(400 \mathrm{MHz}, \mathrm{D}_{2} \mathrm{O}\right) \delta: 7.64$ (br s, 8H), 7.39 (br s, 2H), $7.02(\mathrm{~s}, 2 \mathrm{H}), 4.36(\mathrm{q}, 2 \mathrm{H}, J=7.04 \mathrm{~Hz}), 4.29(\mathrm{dd}, 2 \mathrm{H}, J=9.20,5.20 \mathrm{~Hz}), 4.09-3.98$ (m, 6H), $3.97(\mathrm{~d}, 2 \mathrm{H}, J=5.82 \mathrm{~Hz}), 2.21-2.09(\mathrm{~m}, 4 \mathrm{H}), 2.06-1.94(\mathrm{~m}, 6 \mathrm{H}), 1.88-1.79(\mathrm{~m}, 2 \mathrm{H}), 1.31$ $(\mathrm{d}, 6 \mathrm{H}, J=7.20 \mathrm{~Hz}), 0.87(\mathrm{~d}, 12 \mathrm{H}, J=6.76 \mathrm{~Hz}), 0.80(\mathrm{~d}, 6 \mathrm{H}, J=7.20 \mathrm{~Hz}), 0.78(\mathrm{~d}, 6 \mathrm{H}, J=7.12$ Hz). UV-Vis $\left(\mathrm{H}_{2} \mathrm{O}\right) \lambda / \mathrm{nm}: 352$ (pH 10). MS (ESI) m/z 1348.5 (M+3K-4H) ${ }^{-}$(calc. 1348.3), 1310.8 $(\mathrm{M}+2 \mathrm{~K}-3 \mathrm{H})^{-}\left(\right.$calc. 1310.2), $1233.8(\mathrm{M}-\mathrm{H})^{-}\left(\right.$calc. 1234.3), m/z $654.5(\mathrm{M}+2 \mathrm{~K}-4 \mathrm{H})^{2-}($ calc. 654.7), m/z $635.5(\mathrm{M}+\mathrm{K}-3 \mathrm{H})^{2-}\left(\right.$ calc. 635.7), m/z $616.9(\mathrm{M}-2 \mathrm{H})^{2-}\left(\right.$ calc. 616.2), m/z $423.4(\mathrm{M}+\mathrm{K}-4 \mathrm{H})^{3-}$ (calc. 423.5), m/z $410.8(\mathrm{M}-3 \mathrm{H})^{3-}$ (calc. 410.8), m/z 307.8 (M-4H) ${ }^{4-}$ (calc. 307.9).

OH-VEVLALG-CH2-P-T-P-CH2-GA $\mathbf{C V}_{\mathbf{L}}$ EV-OH: Prepared according to literature procedure and characterization matched that of literature. ${ }^{1}$

OH-VEVLADG-CH2-P-T-P-CH2-GADVLEV-OH: Solid supported Wang-VEV $\mathrm{A}_{\mathrm{D}} \mathrm{G}_{2} \mathrm{NH}_{2}$ peptide $\mathrm{N}$-acylated with 4-bromophenylacetic acid was prepared $(0.10 \mathrm{mmol})$. The resin was subjected to the standard Stille coupling procedure in the presence of 2,5- 
bis(trimethylstannyl)thiophene $(0.050 \mathrm{mmol}, 0.021 \mathrm{~g})$ and $\mathrm{Pd}\left(\mathrm{PPh}_{3}\right)_{4}(0.0040 \mathrm{mmol}, 0.0046 \mathrm{~g})$ for $21 \mathrm{~h}$. Following general cleavage and work-up, the peptide was obtained as a white powder $(0.032$ mmol, $0.040 \mathrm{~g}, 32 \%$ yield). Following HPLC purification, $0.0070 \mathrm{mmol}, 0.088 \mathrm{~g}, 7.0 \%$ yield. ${ }^{1} \mathrm{H}$ NMR (400 MHz, $\left.\mathrm{D}_{2} \mathrm{O}\right)$ ): $7.76(\mathrm{~s}, 4 \mathrm{H}), 7.69(\mathrm{~d}, 4 \mathrm{H}, J=7.60 \mathrm{~Hz}), 4.30-4.24(\mathrm{~m}, 4 \mathrm{H}), 4.03(\mathrm{~d}, 2 \mathrm{H}$, $J=7.60 \mathrm{~Hz}), 3.97(\mathrm{~d}, 2 \mathrm{H}, J=5.84 \mathrm{~Hz}), 3.88(\mathrm{~s}, 4 \mathrm{H}), 3.67(\mathrm{~d}, 4 \mathrm{H}, J=4.20 \mathrm{~Hz}), 2.21-2.09(\mathrm{~m}, 6 \mathrm{H})$, 2.04-1.92 (m, 4H), 1.88-1.78 (m, 2H), $1.27(\mathrm{~d}, 6 \mathrm{H}, J=7.12 \mathrm{~Hz}), 0.79(\mathrm{t}, 12 \mathrm{H}, J=7.44 \mathrm{~Hz}), 0.74$ (d, $12 \mathrm{H}, J=6.92 \mathrm{~Hz})$. UV-Vis $\left(\mathrm{H}_{2} \mathrm{O}\right) \lambda / \mathrm{nm}: 331(\mathrm{pH} \mathrm{10}) . \mathrm{MS}(\mathrm{ESI}) \mathrm{m} / \mathrm{z} 1261.8(\mathrm{M}-\mathrm{H})^{-}$(calc. 1261.6), m/z 649.45 (M+K-2H) ${ }^{2-}$ (calc. 649.90), m/z $630.85(\mathrm{M}-2 \mathrm{H})^{2-}$ (calc. 630.33), m/z 420.11 $(\mathrm{M}-3 \mathrm{H})^{3-}$ (calc. 419.83), m/z $314.84(\mathrm{M}-4 \mathrm{H})^{4-}($ calc. 314.63).

OH-VEV ${ }_{D} A_{L}$ G-CH2-P-T-P-CH2-GALVDEV-OH: Solid supported Wang-VEV ${ }_{D} A_{L} G_{-}-N_{2}$ peptide $\mathrm{N}$-acylated with 4-bromophenylacetic acid was prepared $(0.10 \mathrm{mmol})$. The resin was subjected to the standard Stille coupling procedure in the presence of 2,5bis(trimethylstannyl)thiophene $(0.050 \mathrm{mmol}, 0.021 \mathrm{~g})$ and $\mathrm{Pd}\left(\mathrm{PPh}_{3}\right)_{4}(0.0040 \mathrm{mmol}, 0.0046 \mathrm{~g})$ for $21 \mathrm{~h}$. Following general cleavage and work-up, the peptide was obtained as a white powder $(0.039$ mmol, $0.049 \mathrm{~g}, 39 \%$ yield). Following HPLC purification, $0.0087 \mathrm{mmol}, 0.011 \mathrm{~g}, 8.7 \%$ yield. ${ }^{1} \mathrm{H}$ NMR (400 MHz, $\left.\mathrm{D}_{2} \mathrm{O}\right) \delta: 7.66(\mathrm{~d}, 2 \mathrm{H}, J=8.36 \mathrm{~Hz}), 7.48(\mathrm{~d}, 3 \mathrm{H}, J=8.36 \mathrm{~Hz}), 7.42(\mathrm{~s}, 1 \mathrm{H}), 7.33$ $(\mathrm{d}, 1 \mathrm{H}, J=8.56 \mathrm{~Hz}), 7.17(\mathrm{~d}, 3 \mathrm{H}, J=8.64 \mathrm{~Hz}), 4.31-4.24(\mathrm{~m}, 4 \mathrm{H}), 4.02(\mathrm{dd}, 4 \mathrm{H}, J=7.28,9.76$ Hz), $3.86(\mathrm{~d}, 4 \mathrm{H}, J=6.56 \mathrm{~Hz}), 3.64(\mathrm{~d}, 2 \mathrm{H}, J=3.68 \mathrm{~Hz}), 3.58(\mathrm{~s}, 2 \mathrm{H}), 2.18-2.10(\mathrm{~m}, 4 \mathrm{H}), 2.06-$ $1.91(\mathrm{~m}, 6 \mathrm{H}), 1.87-1.75(\mathrm{~m}, 2 \mathrm{H}), 1.28(\mathrm{~d}, 6 \mathrm{H}, J=7.20 \mathrm{~Hz}), 0.84(\mathrm{~d}, 6 \mathrm{H}, J=6.76 \mathrm{~Hz}), 0.81(\mathrm{~d}, 6 \mathrm{H}$, $J=7.04 \mathrm{~Hz}), 0.79(\mathrm{~d}, 6 \mathrm{H}, J=1.76 \mathrm{~Hz}), 0.76(\mathrm{~d}, 6 \mathrm{H}, J=7.04 \mathrm{~Hz}) . \mathrm{UV}-\mathrm{V}$ is $\left(\mathrm{H}_{2} \mathrm{O}\right) \lambda / \mathrm{nm}: 330(\mathrm{pH}$ 10). MS (ESI) m/z 1261.6 (M-H) (calc. 1261.6), m/z 631.17 (M-2H) $)^{2-}$ (calc. 630.33), m/z 420.22 $(\mathrm{M}-3 \mathrm{H})^{3-}($ calc. 419.83$), \mathrm{m} / \mathrm{z} 334.81(\mathrm{M}+2 \mathrm{~K}-6 \mathrm{H})^{4-}\left(\right.$ calc. 333.65), m/z $314.84(\mathrm{M}-4 \mathrm{H})^{4-}$ (calc. 314.63). 
OH-VEVDADG-CH2-P-T-P-CH2-GADVDEV-OH: Solid supported Wang-VEV ${ }_{D} A_{D} G_{-}-\mathrm{NH}_{2}$ peptide $\mathrm{N}$-acylated with 4-bromophenylacetic acid was prepared $(0.10 \mathrm{mmol})$. The resin was subjected to the standard Stille coupling procedure in the presence of 2,5bis(trimethylstannyl)thiophene $(0.050 \mathrm{mmol}, 0.021 \mathrm{~g})$ and $\mathrm{Pd}\left(\mathrm{PPh}_{3}\right)_{4}(0.0040 \mathrm{mmol}, 0.0046 \mathrm{~g})$ for 21 h. Following general cleavage and work-up, the peptide was obtained as a white powder $(0.042$ mmol, $0.053 \mathrm{~g}, 42 \%$ yield). Following HPLC purification, $0.010 \mathrm{mmol}, 0.013 \mathrm{~g}, 10 \%$ yield. ${ }^{1} \mathrm{H}$

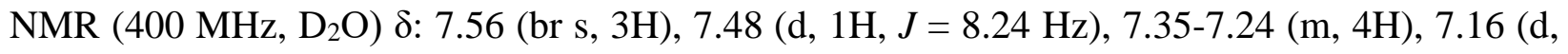
$1 \mathrm{H}, J=8.24 \mathrm{~Hz}), 4.31-4.23(\mathrm{~m}, 4 \mathrm{H}), 4.01(\mathrm{dd}, 2 \mathrm{H}, J=6.56,9.84 \mathrm{~Hz}), 3.98-3.96(\mathrm{~m}, 2 \mathrm{H}), 3.89-$ $3.78(\mathrm{~m}, 4 \mathrm{H}), 3.59(\mathrm{~s}, 2 \mathrm{H}), 3.57(\mathrm{~s}, 2 \mathrm{H}), 2.25-2.09(\mathrm{~m}, 4 \mathrm{H}), 2.06-1.89(\mathrm{~m}, 6 \mathrm{H}), 1.87-1.76(\mathrm{~m}, 2 \mathrm{H})$, $1.23(\mathrm{~d}, 2 \mathrm{H}, J=6.96 \mathrm{~Hz}), 1.22(\mathrm{~d}, 4 \mathrm{H}, J=7.16 \mathrm{~Hz}), 0.85(\mathrm{~d}, 6 \mathrm{H}, J=6.88 \mathrm{~Hz}), 0.80(\mathrm{~d}, 12 \mathrm{H}, J=$ $7.16 \mathrm{~Hz}), 0.77(\mathrm{~d}, 6 \mathrm{H}, J=6.84 \mathrm{~Hz}) . \mathrm{UV}-\mathrm{Vis}\left(\mathrm{H}_{2} \mathrm{O}\right) \lambda / \mathrm{nm}: 331(\mathrm{pH} 10) . \mathrm{MS}(\mathrm{ESI}) \mathrm{m} / \mathrm{z} 1261.3$ (MH) ${ }^{-}$(calc. 1261.6), m/z $630.70(\mathrm{M}-2 \mathrm{H})^{2-}\left(\right.$ calc. 630.33), m/z $420.20(\mathrm{M}-3 \mathrm{H})^{3-}$ (calc. 419.83), m/z $334.49(\mathrm{M}+2 \mathrm{~K}-6 \mathrm{H})^{4-}\left(\right.$ calc. 333.65), m/z $314.72(\mathrm{M}-4 \mathrm{H})^{4-}($ calc. 314.63)

OH-VEVLALG-C2 $\mathrm{H}_{4}-\mathrm{P}-\mathrm{T}-\mathrm{P}-\mathrm{C}_{2} \mathrm{H}_{4}-\mathrm{GA}_{L} \mathrm{VLEV}_{\mathrm{L}} \mathrm{-OH}$ : Prepared according to literature procedure and characterization matched that of literature. ${ }^{1}$

OH-VEVLADG-C2 $H_{4}-P-T-P-C_{2} H_{4}-G_{D} V_{L}$ EV-OH: Solid supported Wang-VEV $A_{D} A_{-N H}$ peptide $\mathrm{N}$-acylated with 3-(4-bromophenyl)propionic acid was prepared $(0.10 \mathrm{mmol})$. The resin was subjected to the standard Stille coupling procedure in the presence of 2,5bis(trimethylstannyl)thiophene $(0.050 \mathrm{mmol}, 0.021 \mathrm{~g})$ and $\mathrm{Pd}\left(\mathrm{PPh}_{3}\right)_{4}(0.0040 \mathrm{mmol}, 0.0046 \mathrm{~g})$ for 21 h. Following general cleavage and work-up, the peptide was obtained as a white powder (0.039 mmol, $0.050 \mathrm{~g}, 39 \%$ yield). Following HPLC purification, $0.011 \mathrm{mmol}, 0.014 \mathrm{~g}, 11 \%$ yield. ${ }^{1} \mathrm{H}$ NMR (400 MHz, D $2 \mathrm{O}) \delta: 7.60(\mathrm{~d}, 4 \mathrm{H}, J=7.16 \mathrm{~Hz}), 7.27$ (br s, 2H), $7.26(\mathrm{~d}, 4 \mathrm{H}, J=7.68 \mathrm{~Hz})$, 4.29-4.19 (m, 4H), $4.03(\mathrm{~d}, 2 \mathrm{H}, J=7.16 \mathrm{~Hz}), 3.96(\mathrm{~d}, 2 \mathrm{H}, J=5.88 \mathrm{~Hz}), 3.77(\mathrm{~s}, 4 \mathrm{H}), 2.91(\mathrm{t}, 4 \mathrm{H}$, 
$J=7.32 \mathrm{~Hz}), 2.62(\mathrm{t}, 4 \mathrm{H}, J=7.52 \mathrm{~Hz}), 2.21-2.08(\mathrm{~m}, 4 \mathrm{H}), 2.04-1.91(\mathrm{~m}, 6 \mathrm{H}), 1.88-1.77(\mathrm{~m}, 2 \mathrm{H})$, $1.28(\mathrm{~d}, 2 \mathrm{H}, J=7.16 \mathrm{~Hz}), 1.22(\mathrm{~d}, 4 \mathrm{H}, J=7.20 \mathrm{~Hz}), 0.81(\mathrm{~d}, 6 \mathrm{H}, J=6.84 \mathrm{~Hz}), 0.80(\mathrm{~d}, 12 \mathrm{H}, J=$ $6.96 \mathrm{~Hz}), 0.77$ (d, 6H, $J=5.84 \mathrm{~Hz})$. UV-Vis $\left(\mathrm{H}_{2} \mathrm{O}\right) \lambda / \mathrm{nm}: 331$ (pH 10). MS (ESI) m/z $1289.6(\mathrm{M}-$ H) ${ }^{-}($calc. 1289.5$), \mathrm{m} / \mathrm{z} 644.86(\mathrm{M}-2 \mathrm{H})^{2-}($ calc. 644.38$), \mathrm{m} / \mathrm{z} 429.51(\mathrm{M}-3 \mathrm{H})^{3-}($ calc. 429.16$), \mathrm{m} / \mathrm{z}$ $321.85(\mathrm{M}-4 \mathrm{H})^{4-}($ calc. 321.65).

OH-VEV DA $A_{L}$ G-C C $_{4}$-P-T-P-C $2 H_{4}-G_{L} V_{D}$ EV-OH: Solid supported Wang-VEV ${ }_{D} A_{L} G-N_{2}$ peptide $\mathrm{N}$-acylated with 3-(4-bromophenyl)propionic acid was prepared $(0.10 \mathrm{mmol})$. The resin was subjected to the standard Stille coupling procedure in the presence of 2,5bis(trimethylstannyl)thiophene $(0.050 \mathrm{mmol}, 0.021 \mathrm{~g})$ and $\mathrm{Pd}\left(\mathrm{PPh}_{3}\right)_{4}(0.0040 \mathrm{mmol}, 0.0046 \mathrm{~g})$ for $21 \mathrm{~h}$. Following general cleavage and work-up, the peptide was obtained as a white powder $(0.032$ mmol, $0.041 \mathrm{~g}, 32 \%$ yield). Following HPLC purification, $0.0085 \mathrm{mmol}, 0.011 \mathrm{~g}, 8.5 \%$ yield. ${ }^{1} \mathrm{H}$ NMR (400 MHz, $\left.\mathrm{D}_{2} \mathrm{O}\right) \delta: 7.57$ (br s, 2H), $7.42(\mathrm{~d}, 2 \mathrm{H}, J=7.64 \mathrm{~Hz}), 7.24(\mathrm{br} \mathrm{s}, 4 \mathrm{H}), 7.10(\mathrm{~d}, 2 \mathrm{H}$, $J=8.40 \mathrm{~Hz}), 4.31-4.14(\mathrm{~m}, 4 \mathrm{H}), 4.09-3.98(\mathrm{~m}, 2 \mathrm{H}), 3.97(\mathrm{~d}, 2 \mathrm{H}, J=6.72 \mathrm{~Hz}), 3.84-3.70(\mathrm{~m}, 4 \mathrm{H})$, $2.84(\mathrm{t}, 4 \mathrm{H}, J=7.92 \mathrm{~Hz}), 2.56(\mathrm{t}, 4 \mathrm{H}, J=7.96 \mathrm{~Hz}), 2.20-2.07(\mathrm{~m}, 4 \mathrm{H}), 2.04-1.90(\mathrm{~m}, 6 \mathrm{H}), 1.88-$ $1.76(\mathrm{~m}, 2 \mathrm{H}), 1.28(\mathrm{~d}, 1 \mathrm{H}, J=6.88 \mathrm{~Hz}), 1.18(\mathrm{br} \mathrm{s}, 5 \mathrm{H}), 0.86(\mathrm{~d}, 6 \mathrm{H}, J=6.76 \mathrm{~Hz}), 0.85-0.71(\mathrm{~m}$, 18H). UV-Vis $\left(\mathrm{H}_{2} \mathrm{O}\right) \lambda / \mathrm{nm}: 332$ (pH 10). MS (ESI) m/z $1403.6(\mathrm{M}+3 \mathrm{~K}-4 \mathrm{H})^{-}$(calc. 1403.7), $\mathrm{m} / \mathrm{z}$

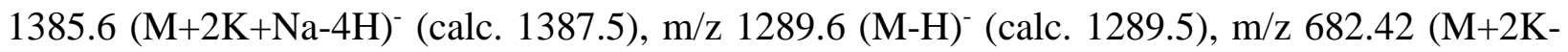
$4 \mathrm{H})^{2-}\left(\right.$ calc. 682.36), m/z $644.85(\mathrm{M}-2 \mathrm{H})^{2-}\left(\right.$ calc. 644.38), m/z $429.48(\mathrm{M}-3 \mathrm{H})^{3-}($ calc. 429.16$), \mathrm{m} / \mathrm{z}$ $321.79(\mathrm{M}-4 \mathrm{H})^{4-}($ calc. 321.65).

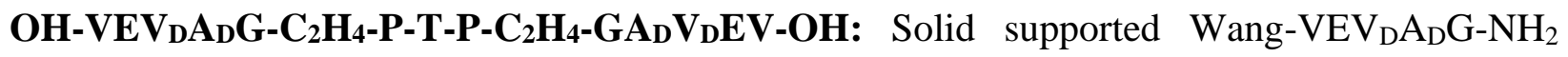
peptide $\mathrm{N}$-acylated with 3-(4-bromophenyl)propionic acid was prepared $(0.10 \mathrm{mmol})$. The resin was subjected to the standard Stille coupling procedure in the presence of 2,5bis(trimethylstannyl)thiophene $(0.050 \mathrm{mmol}, 0.021 \mathrm{~g})$ and $\mathrm{Pd}\left(\mathrm{PPh}_{3}\right)_{4}(0.0040 \mathrm{mmol}, 0.0046 \mathrm{~g})$ for 
$21 \mathrm{~h}$. Following general cleavage and work-up, the peptide was obtained as a white powder $(0.046$ mmol, $0.059 \mathrm{~g}, 46 \%$ yield). Following HPLC purification, $0.015 \mathrm{mmol}, 0.019 \mathrm{~g}, 15 \%$ yield. ${ }^{1} \mathrm{H}$ NMR (400 MHz, $\left.\mathrm{D}_{2} \mathrm{O}\right)$ 8: 7.56 (br s, 2H), $7.43(\mathrm{~d}, 2 \mathrm{H}, J=7.92 \mathrm{~Hz}), 7.33$ (br s, 2H), 7.23 (br s, $2 \mathrm{H}), 7.11(\mathrm{~d}, 2 \mathrm{H}, J=8.36 \mathrm{~Hz}) 4.33-4.19(\mathrm{~m}, 4 \mathrm{H}), 4.08-3.96(\mathrm{~m}, 4 \mathrm{H}), 3.75(\mathrm{~d}, 4 \mathrm{H}, J=5.88 \mathrm{~Hz})$, $3.74(\mathrm{~d}, 2 \mathrm{H}, J=6.92 \mathrm{~Hz}), 3.77-3.64(\mathrm{~m}, 4 \mathrm{H}), 2.89(\mathrm{br} \mathrm{s}, 2 \mathrm{H}), 2.84(\mathrm{t}, 2 \mathrm{H}, J=7.88 \mathrm{~Hz}), 2.60(\mathrm{br} \mathrm{s}$, 2H), $2.56(\mathrm{t}, 2 \mathrm{H}, J=7.92 \mathrm{~Hz}), 2.24-2.12(\mathrm{~m}, 4 \mathrm{H}), 2.07-1.92(\mathrm{~m}, 6 \mathrm{H}), 1.89-1.78(\mathrm{~m}, 2 \mathrm{H}), 1.25(\mathrm{~d}$, $4 \mathrm{H}, J=6.96 \mathrm{~Hz}), 1.19(\mathrm{~d}, 2 \mathrm{H}, J=7.20 \mathrm{~Hz}), 0.87(\mathrm{~d}, 6 \mathrm{H}, J=6.64 \mathrm{~Hz}), 0.85-0.78(\mathrm{~m}, 12 \mathrm{H}), 0.76$ (d, $6 \mathrm{H}, J=6.64 \mathrm{~Hz})$. UV-Vis $\left(\mathrm{H}_{2} \mathrm{O}\right) \lambda / \mathrm{nm}: 330(\mathrm{pH} 10)$. MS (ESI) m/z $1404.3(\mathrm{M}+3 \mathrm{~K}-4 \mathrm{H})^{-}{ }^{-}$calc. 1403.7), m/z $1385.5(\mathrm{M}+2 \mathrm{~K}+\mathrm{Na}-4 \mathrm{H})^{-}$(calc. 1387.5), m/z $1289.8(\mathrm{M}-\mathrm{H})^{-}$(calc. 1289.5), m/z $682.39(\mathrm{M}+2 \mathrm{~K}-4 \mathrm{H})^{2-}\left(\right.$ calc. 682.36), m/z $663.55(\mathrm{M}+\mathrm{K}-3 \mathrm{H})^{2-}\left(\right.$ calc. 663.25), m/z $644.85(\mathrm{M}-2 \mathrm{H})^{2-}$

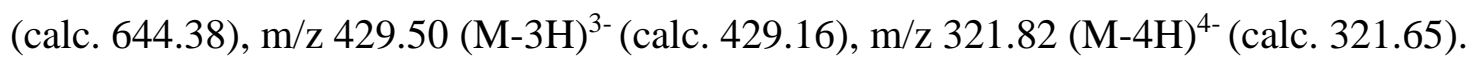

OH-VEV LALG-C $_{3} \mathrm{H}_{6}-\mathbf{P}-\mathrm{T}-\mathbf{P}-\mathrm{C}_{3} \mathrm{H}_{6}-\mathrm{GA}_{\mathrm{L}} \mathrm{V}_{\mathrm{L}} \mathrm{EV}-\mathrm{OH}$ : Prepared according to literature procedure and characterization matched that of literature. ${ }^{1}$

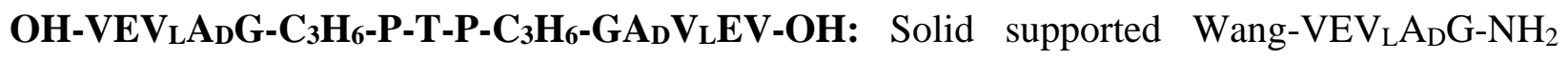
peptide $\mathrm{N}$-acylated with 4-(4-bromophenyl)butanoic acid was prepared $(0.10 \mathrm{mmol})$. The resin was subjected to the standard Stille coupling procedure in the presence of 2,5bis(trimethylstannyl)thiophene $(0.050 \mathrm{mmol}, 0.021 \mathrm{~g})$ and $\mathrm{Pd}\left(\mathrm{PPh}_{3}\right)_{4}(0.0040 \mathrm{mmol}, 0.0046 \mathrm{~g})$ for $21 \mathrm{~h}$. Following general cleavage and work-up, the peptide was obtained as a white powder $(0.036$ mmol, $0.047 \mathrm{~g}, 36 \%$ yield). Following HPLC purification, $0.0076 \mathrm{mmol}, 0.010 \mathrm{~g}, 7.6 \%$ yield. ${ }^{1} \mathrm{H}$ NMR (400 MHz, $\left.\mathrm{D}_{2} \mathrm{O}\right) \delta: 7.65-7.47(\mathrm{~m}, 6 \mathrm{H}), 7.36(\mathrm{~s}, 2 \mathrm{H}), 7.25(\mathrm{~d}, 2 \mathrm{H}, J=8.36 \mathrm{~Hz}), 4.31-4.24$ (m, 4H), $4.01(\mathrm{q}, 2 \mathrm{H}, J=3.76 \mathrm{~Hz}), 3.96(\mathrm{~d}, 2 \mathrm{H}, J=5.88 \mathrm{~Hz}), 3.75(\mathrm{~d}, 2 \mathrm{H}, J=6.04 \mathrm{~Hz}), 3.72-3.68$ (m, 1H), $2.67(\mathrm{t}, 2 \mathrm{H}, J=7.32 \mathrm{~Hz}), 2.61(\mathrm{t}, 2 \mathrm{H}, J=7.24 \mathrm{~Hz}), 2.29-2.20(\mathrm{~m}, 4 \mathrm{H}), 2.20-2.05(\mathrm{~m}, 6 \mathrm{H})$, 2.04-1.91 (m, 6H), $1.88(\mathrm{t}, 3 \mathrm{H}, J=6.92 \mathrm{~Hz}), 1.85-1.76(\mathrm{~m}, 3 \mathrm{H}), 1.24(\mathrm{~d}, 6 \mathrm{H}, J=7.16 \mathrm{~Hz}), 0.84$ 
$(\mathrm{d}, 6 \mathrm{H}, J=6.76 \mathrm{~Hz}), 0.81(\mathrm{~d}, 6 \mathrm{H}, J=6.76 \mathrm{~Hz}), 0.77(\mathrm{~d}, 12 \mathrm{H}, J=6.84 \mathrm{~Hz})$. UV-Vis $\left(\mathrm{H}_{2} \mathrm{O}\right) \lambda / \mathrm{nm}$ : 331 (pH 10). MS (ESI) m/z 1393.5 (M+2K-3H)- (calc. 1394.4), m/z 1317.6 (M-H) (calc. 1318.4), m/z 696.51 (M+2K-4H) $)^{2-}\left(\right.$ calc. 696.73), m/z 659.11 (M-2H) ${ }^{2-}$ (calc. 658.77).

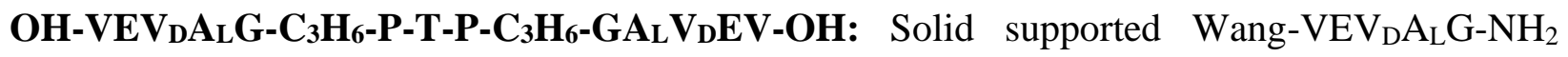
peptide $\mathrm{N}$-acylated with 4-(4-bromophenyl)butanoic acid was prepared $(0.10 \mathrm{mmol})$. The resin was subjected to the standard Stille coupling procedure in the presence of 2,5bis(trimethylstannyl)thiophene $(0.050 \mathrm{mmol}, 0.021 \mathrm{~g})$ and $\mathrm{Pd}\left(\mathrm{PPh}_{3}\right)_{4}(0.0040 \mathrm{mmol}, 0.0046 \mathrm{~g})$ for $21 \mathrm{~h}$. Following general cleavage and work-up, the peptide was obtained as a white powder $(0.033$ mmol, $0.043 \mathrm{~g}, 33 \%$ yield). Following HPLC purification, $0.0052 \mathrm{mmol}, 0.0068 \mathrm{~g}, 5.2 \%$ yield. ${ }^{1} \mathrm{H}$ NMR (400 MHz, $\left.\mathrm{D}_{2} \mathrm{O}\right) \delta: 7.71(\mathrm{~s}, 3 \mathrm{H}), 7.63(\mathrm{~d}, 3 \mathrm{H}, J=8.12 \mathrm{~Hz}), 7.32(\mathrm{~d}, 3 \mathrm{H}, J=8.08 \mathrm{~Hz}), 4.30-$ $4.20(\mathrm{~m}, 4 \mathrm{H}), 4.01(\mathrm{~d}, 2 \mathrm{H}, J=7.64 \mathrm{~Hz}), 3.96(\mathrm{~d}, 2 \mathrm{H}, J=5.80 \mathrm{~Hz}), 3.77(\mathrm{~d}, 4 \mathrm{H}, J=6.76 \mathrm{~Hz}), 2.93$ (t, 4H, $J=7.16 \mathrm{~Hz}), 2.62(\mathrm{t}, 4 \mathrm{H}, J=7.16 \mathrm{~Hz}), 2.20-2.11(\mathrm{~m}, 4 \mathrm{H}), 2.05-1.91(\mathrm{~m}, 6 \mathrm{H}), 1.87-1.77$ $(\mathrm{m}, 3 \mathrm{H}), 1.24(\mathrm{~d}, 1 \mathrm{H}, J=7.08 \mathrm{~Hz}), 1.20(\mathrm{~d}, 4 \mathrm{H}, J=7.28 \mathrm{~Hz}), 0.84(\mathrm{~d}, 12 \mathrm{H}, J=6.72 \mathrm{~Hz}), 0.77(\mathrm{t}$, $12 \mathrm{H}, J=6.92 \mathrm{~Hz})$. UV-Vis $\left(\mathrm{H}_{2} \mathrm{O}\right) \lambda / \mathrm{nm}: 332(\mathrm{pH} \mathrm{10})$. MS (ESI) m/z $1393.5(\mathrm{M}+2 \mathrm{~K}-3 \mathrm{H})$ - (calc. 1394.4), m/z $1355.6(\mathrm{M}+\mathrm{K}-2 \mathrm{H})^{2-}$ (calc. 1355.8), m/z $1317.8(\mathrm{M}-\mathrm{H})^{-}$(calc. 1318.4), m/z 677.68 $(\mathrm{M}+\mathrm{K}-3 \mathrm{H})^{2-}($ calc. 677.56$), \mathrm{m} / \mathrm{z} 659.11(\mathrm{M}-2 \mathrm{H})^{2-}($ calc. 658.77).

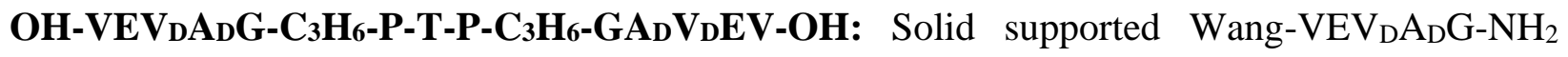
peptide $\mathrm{N}$-acylated with 4-(4-bromophenyl)butanoic acid was prepared $(0.10 \mathrm{mmol})$. The resin was subjected to the standard Stille coupling procedure in the presence of 2,5bis(trimethylstannyl)thiophene $(0.050 \mathrm{mmol}, 0.021 \mathrm{~g})$ and $\mathrm{Pd}\left(\mathrm{PPh}_{3}\right)_{4}(0.0040 \mathrm{mmol}, 0.0046 \mathrm{~g})$ for $21 \mathrm{~h}$. Following general cleavage and work-up, the peptide was obtained as a white powder $(0.039$ mmol, $0.051 \mathrm{~g}, 39 \%$ yield). Following HPLC purification, $0.0083 \mathrm{mmol}, 0.011 \mathrm{~g}, 8.3 \%$ yield. ${ }^{1} \mathrm{H}$ NMR (400 MHz, $\left.\mathrm{D}_{2} \mathrm{O}\right)$ 8: 7.56-7.50 (m, 1H), 7.46-7.31 (m, 3H), 7.23 (br s, 3H), 6.95 (br s, 3H), 
4.28-4.19 (m, 4H), $3.98(\mathrm{~d}, 2 \mathrm{H}, J=7.56 \mathrm{~Hz}), 3.91(\mathrm{~d}, 2 \mathrm{H}, J=6.48 \mathrm{~Hz}), 3.74(\mathrm{~d}, 2 \mathrm{H}, J=6.92 \mathrm{~Hz})$, 3.71-3.41 (m, 2H), $2.54(\mathrm{t}, 1 \mathrm{H}, J=6.76 \mathrm{~Hz}), 2.41(\mathrm{br} \mathrm{s}, 3 \mathrm{H}), 2.20-2.03(\mathrm{~m}, 8 \mathrm{H}), 1.99-1.85(\mathrm{~m}, 6 \mathrm{H})$, $1.83-1.68(\mathrm{~m}, 6 \mathrm{H}), 1.22(\mathrm{~d}, 6 \mathrm{H}, J=7.16 \mathrm{~Hz}), 0.78-0.75(\mathrm{~m}, 12 \mathrm{H}), 0.73(\mathrm{~d}, 12 \mathrm{H}, J=6.72 \mathrm{~Hz}) . \mathrm{UV}-$ Vis $\left(\mathrm{H}_{2} \mathrm{O}\right) \lambda / \mathrm{nm}: 331$ (pH 10). MS (ESI) m/z 1393.5 (M+2K-3H)- (calc. 1394.4), m/z 1355.6 $(\mathrm{M}+\mathrm{K}-2 \mathrm{H})^{2-}\left(\right.$ calc. 1355.8), m/z $1317.8(\mathrm{M}-\mathrm{H})^{-}\left(\right.$calc. 1318.4), m/z $659.03(\mathrm{M}-2 \mathrm{H})^{2-}($ calc. 658.77).

OH-V $\mathbf{D E V}_{\mathbf{L}} \mathbf{A}_{\mathbf{L}}$ G-P-T-P-GA $\mathbf{V}_{\mathbf{L}} \mathbf{E V}_{\mathbf{D}}-\mathrm{OH}$ : Solid supported Wang-V $\mathrm{D}_{\mathrm{D}} \mathrm{EVAG}^{-\mathrm{NH}_{2}}$ peptide Nacylated with 4-iodobenzoic acid was prepared $(0.10 \mathrm{mmol})$. The resin was subjected to the standard Stille coupling procedure in the presence of 2,5-bis(trimethylstannyl)thiophene $(0.050$ mmol, $0.021 \mathrm{~g})$ and $\mathrm{Pd}\left(\mathrm{PPh}_{3}\right)_{4}(0.0040 \mathrm{mmol}, 0.0046 \mathrm{~g})$ for $21 \mathrm{~h}$. Following general cleavage and work-up, the peptide was obtained as a white powder $(0.052 \mathrm{mmol}, 0.064 \mathrm{~g}, 52 \%$ yield $)$. Following HPLC purification, $0.017 \mathrm{mmol}, 0.021 \mathrm{~g}, 17 \%$ yield. ${ }^{1} \mathrm{H}$ NMR (400 MHz, $\left.\mathrm{D}_{2} \mathrm{O}\right) \delta: 7.79(\mathrm{~s}, 8 \mathrm{H})$, $7.54(\mathrm{~s}, 2 \mathrm{H}), 4.36(\mathrm{q}, 2 \mathrm{H}, J=7.04 \mathrm{~Hz}), 4.29(\mathrm{dd}, 2 \mathrm{H}, J=8.72,4.88 \mathrm{~Hz}), 4.12-4.03(\mathrm{~m}, 5 \mathrm{H}), 4.01$ $(\mathrm{d}, 2 \mathrm{H}, J=5.64 \mathrm{~Hz}), 2.21-2.14(\mathrm{~m}, 4 \mathrm{H}), 2.06-1.93(\mathrm{~m}, 6 \mathrm{H}), 1.90-1.79(\mathrm{~m}, 2 \mathrm{H}), 1.33(\mathrm{~d}, 6 \mathrm{H}, J=$ $7.16 \mathrm{~Hz}), 0.87(\mathrm{~d}, 12 \mathrm{H}, J=6.84 \mathrm{~Hz}), 0.82(\mathrm{~d}, 6 \mathrm{H}, J=6.92 \mathrm{~Hz}), 0.78(\mathrm{~d}, 6 \mathrm{H}, J=6.72 \mathrm{~Hz}) . \quad \mathrm{UV}-$ Vis $\left(\mathrm{H}_{2} \mathrm{O}\right) \lambda / \mathrm{nm}: 352$ (pH 10). MS (ESI) m/z $1348.5(\mathrm{M}+3 \mathrm{~K}-4 \mathrm{H})^{-}$(calc. 1348.3), $1310.8(\mathrm{M}+2 \mathrm{~K}-$ $3 \mathrm{H})^{-}\left(\right.$calc. 1310.2), 1233.8 (M-H)- (calc. 1234.3), m/z 654.5 (M+2K-4H) ${ }^{2-}($ calc. 654.7), m/z 635.5 $(\mathrm{M}+\mathrm{K}-3 \mathrm{H})^{2-}\left(\right.$ calc. 635.7), m/z $616.9(\mathrm{M}-2 \mathrm{H})^{2-}\left(\right.$ calc. 616.2), m/z $423.4(\mathrm{M}+\mathrm{K}-4 \mathrm{H})^{3-}($ calc. 423.5), $\mathrm{m} / \mathrm{z} 410.8(\mathrm{M}-3 \mathrm{H})^{3-}\left(\right.$ calc. 410.8), m/z $307.8(\mathrm{M}-4 \mathrm{H})^{4-}$ (calc. 307.9). 


\section{ESI Spectra}

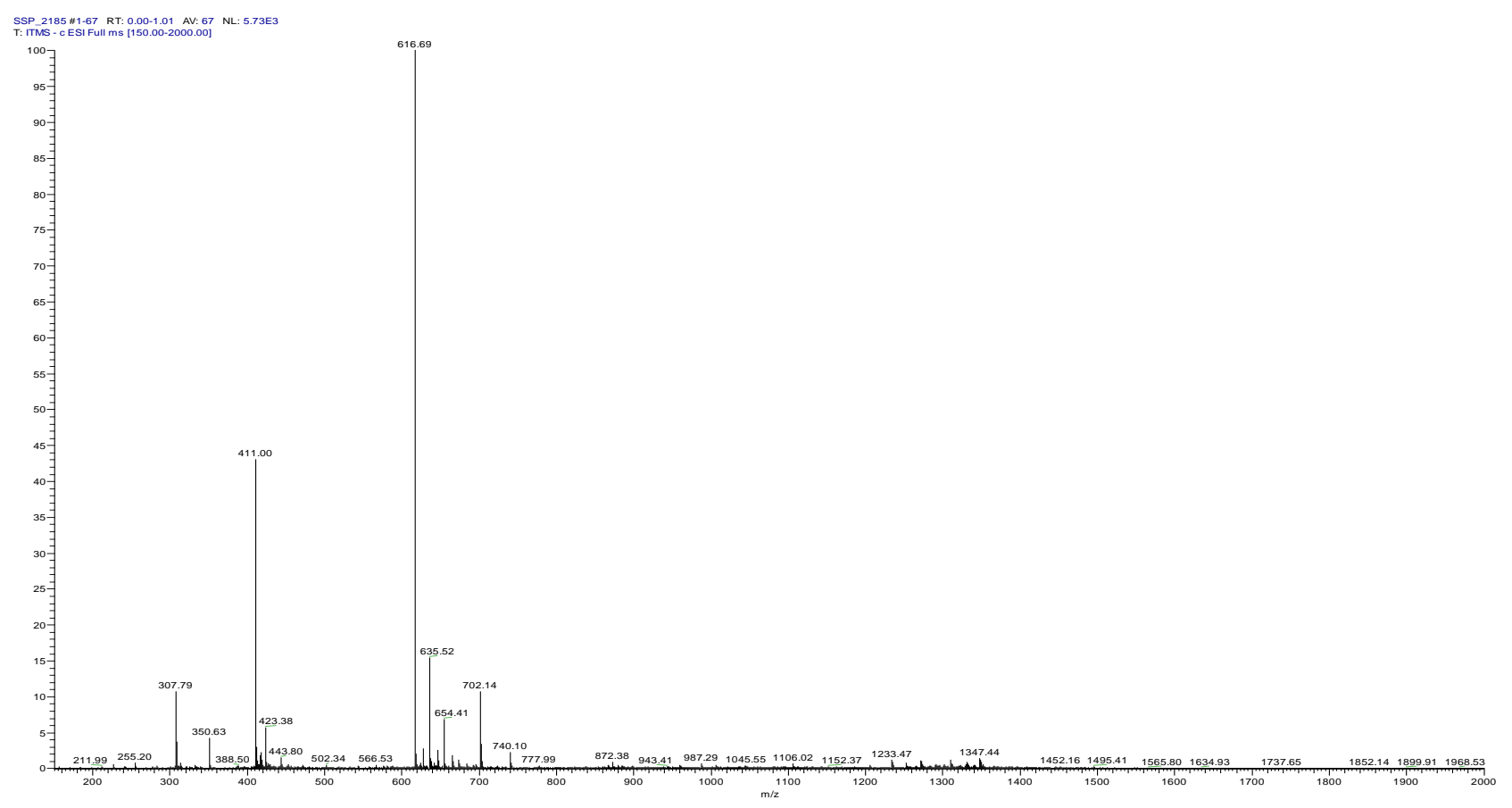

Figure S1. ESI- of $V_{L} A_{D}-0$.

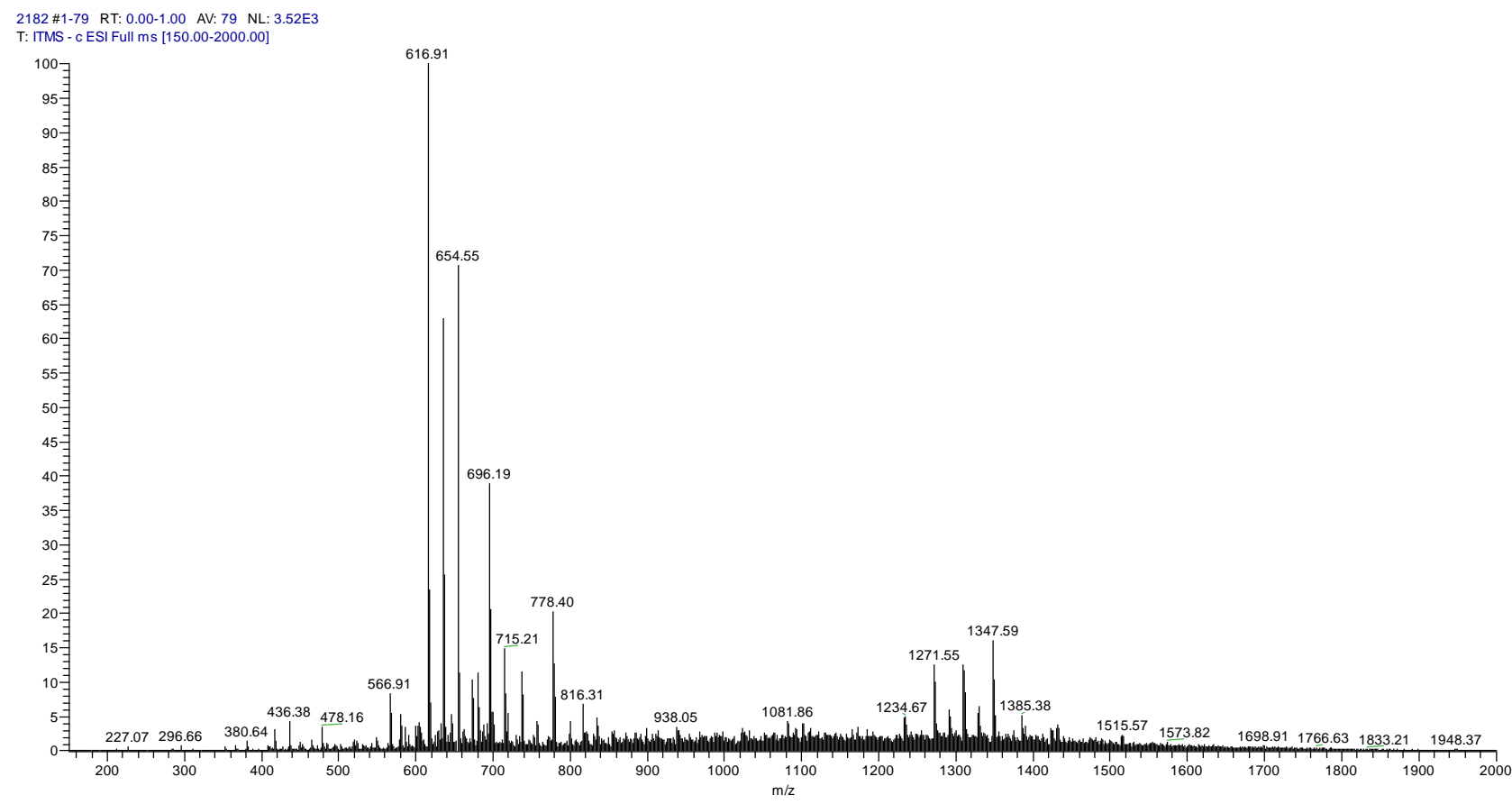

Figure S2. ESI- of $V_{D A} A_{L-0}$. 


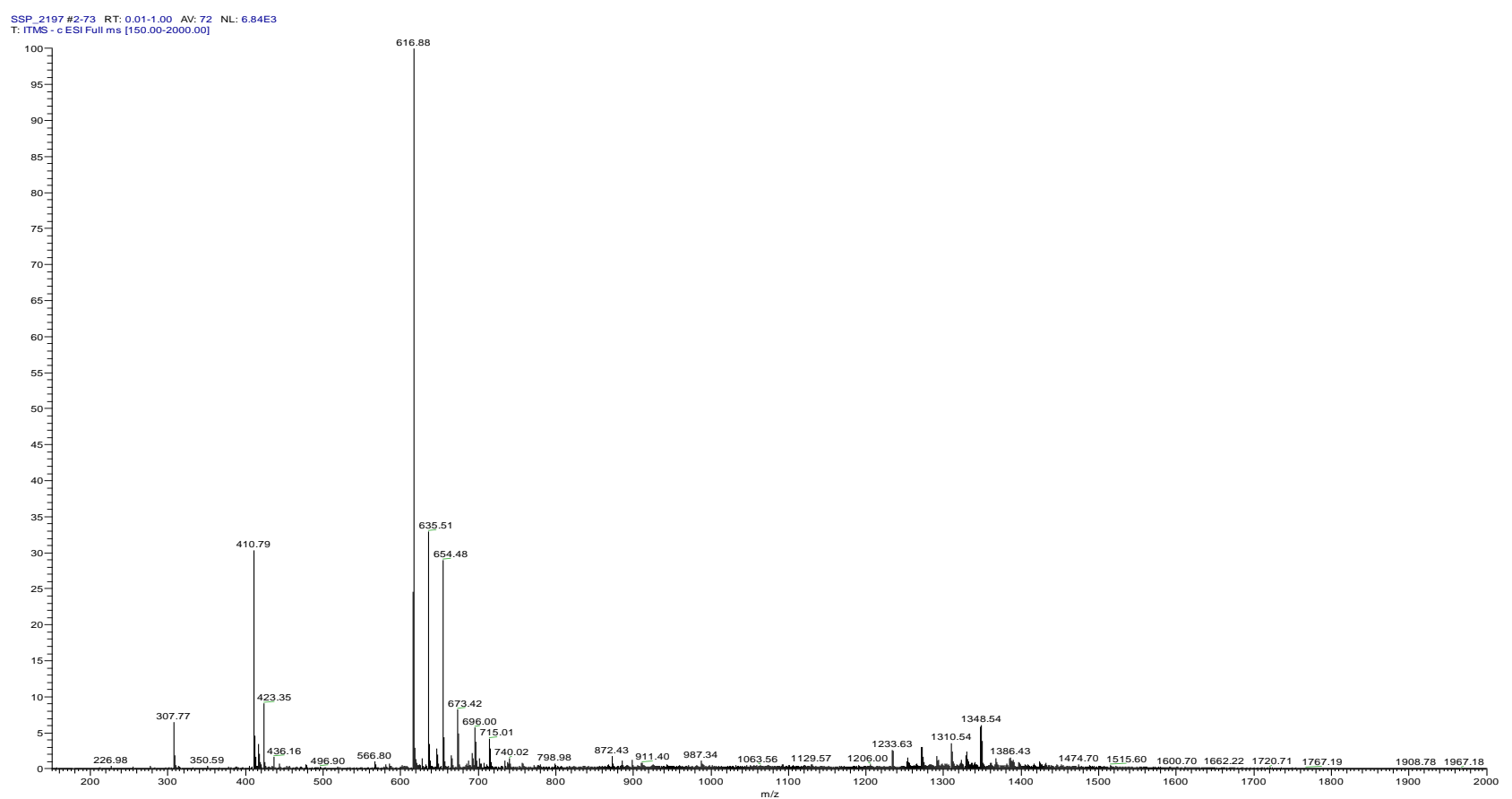

Figure S3. ESI- of VDAd-0.

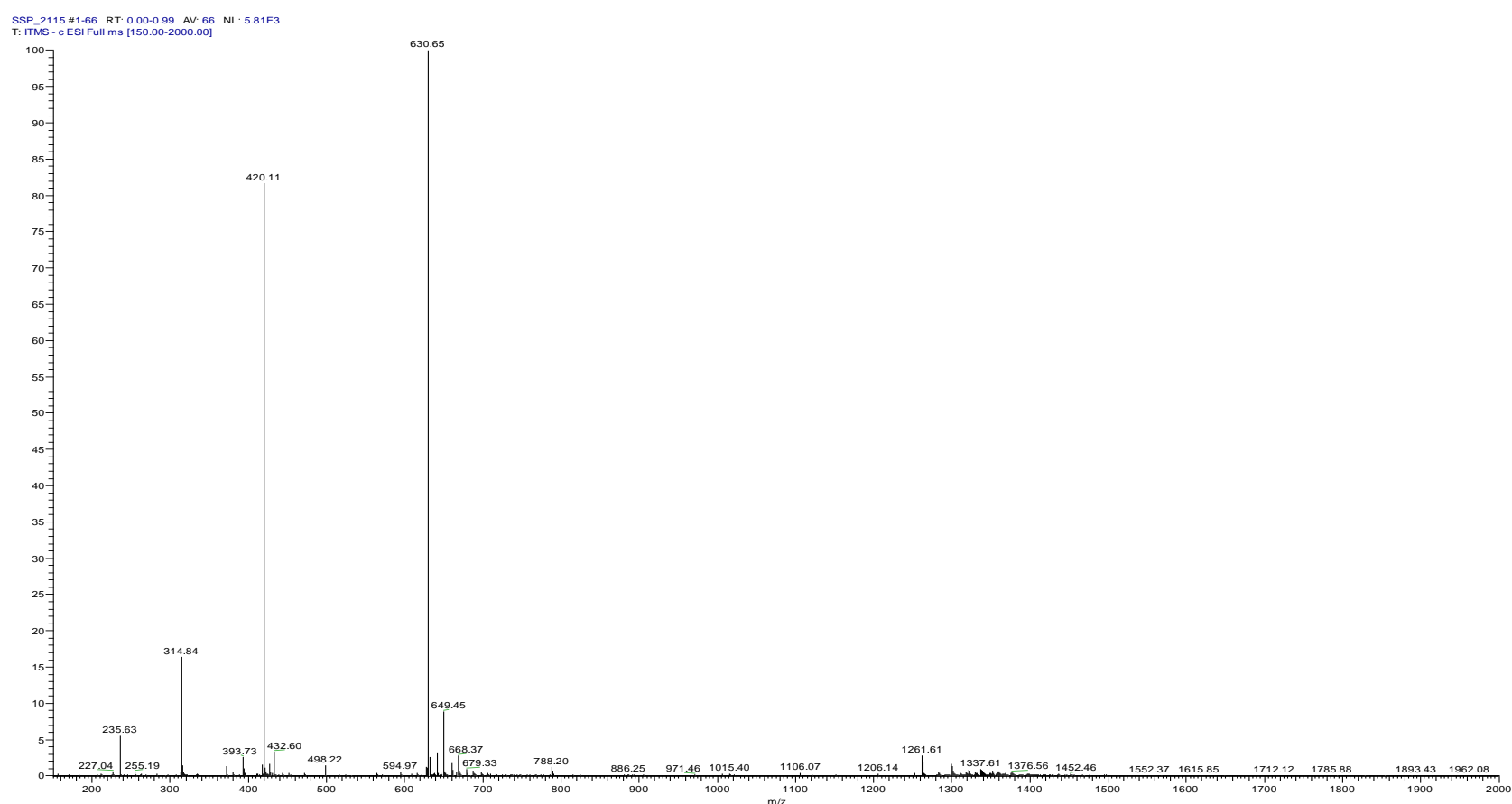

Figure S4. ESI- of VLAd-1. 


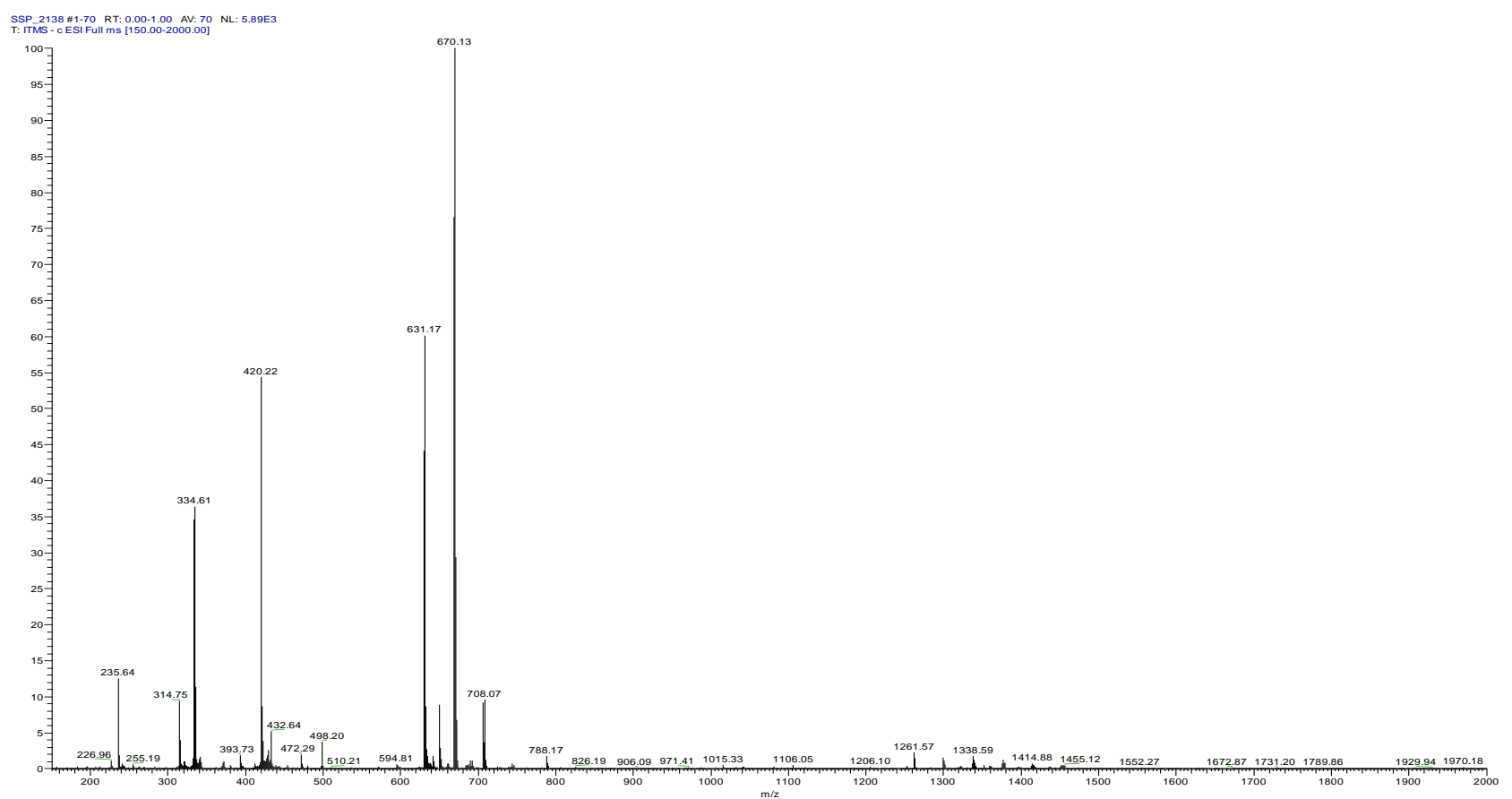

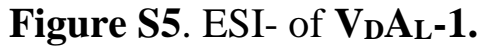

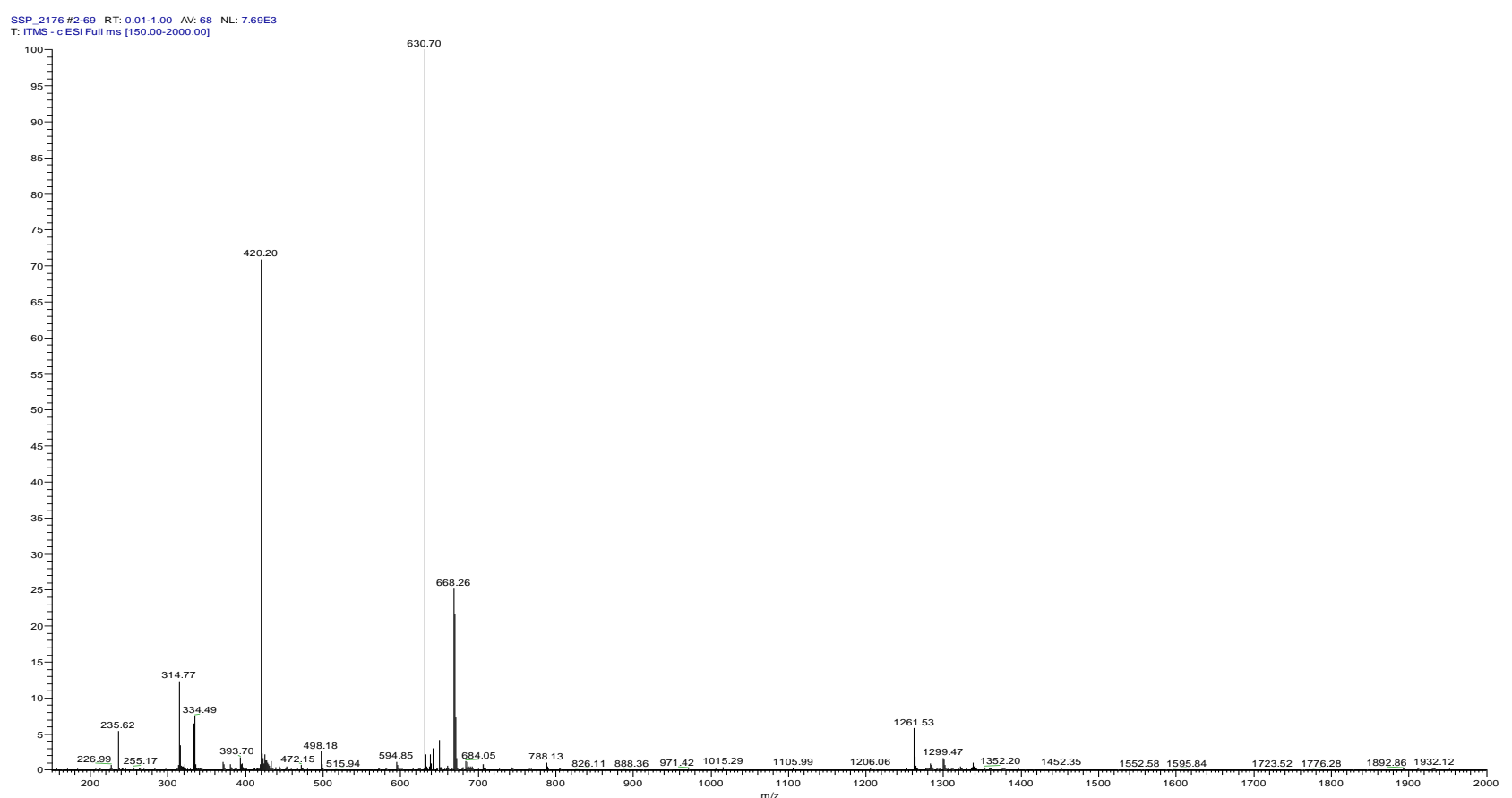

Figure S6. ESI- of VDAD-1. 


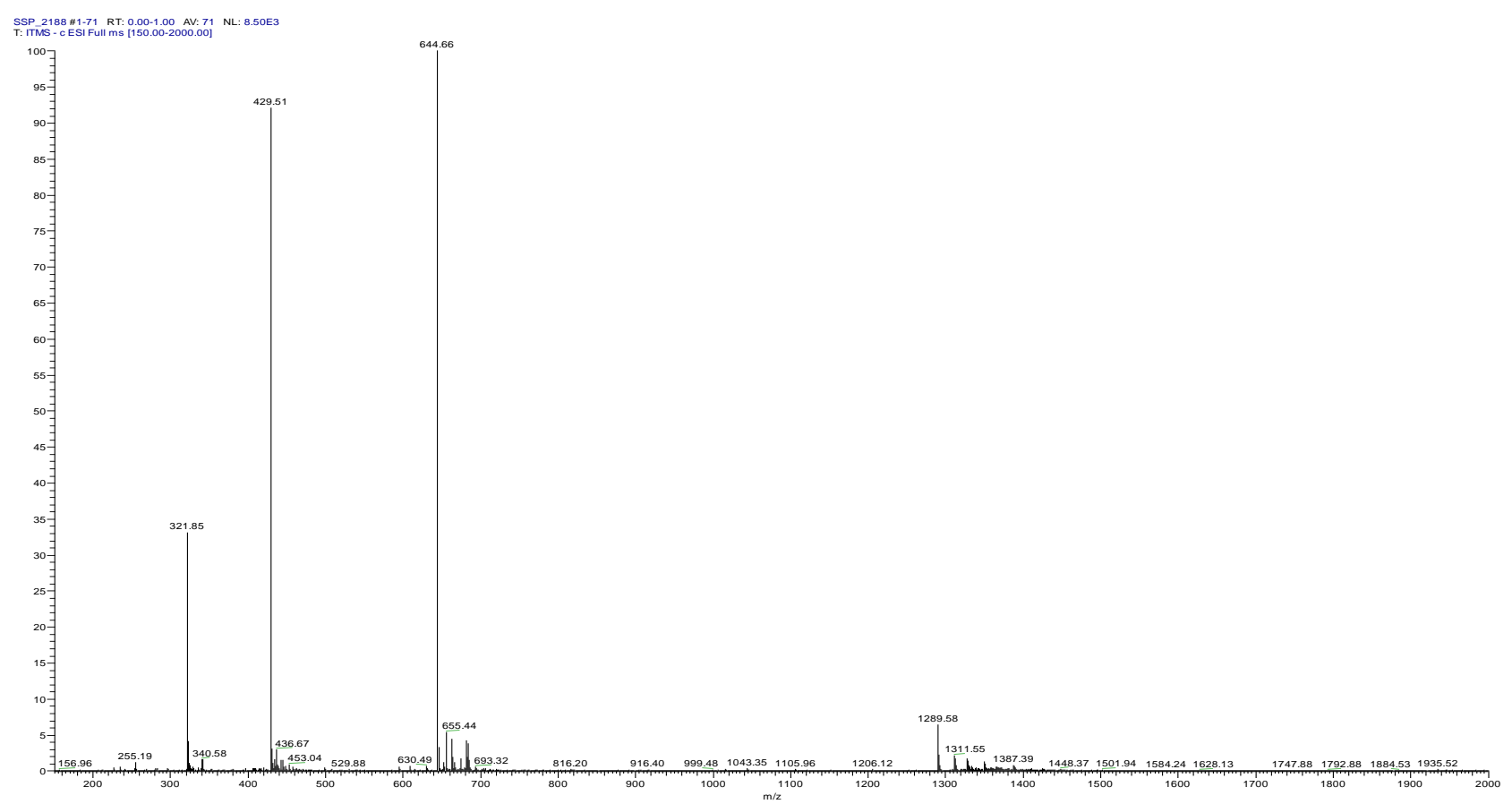

Figure S7. ESI- of VLAD-2.

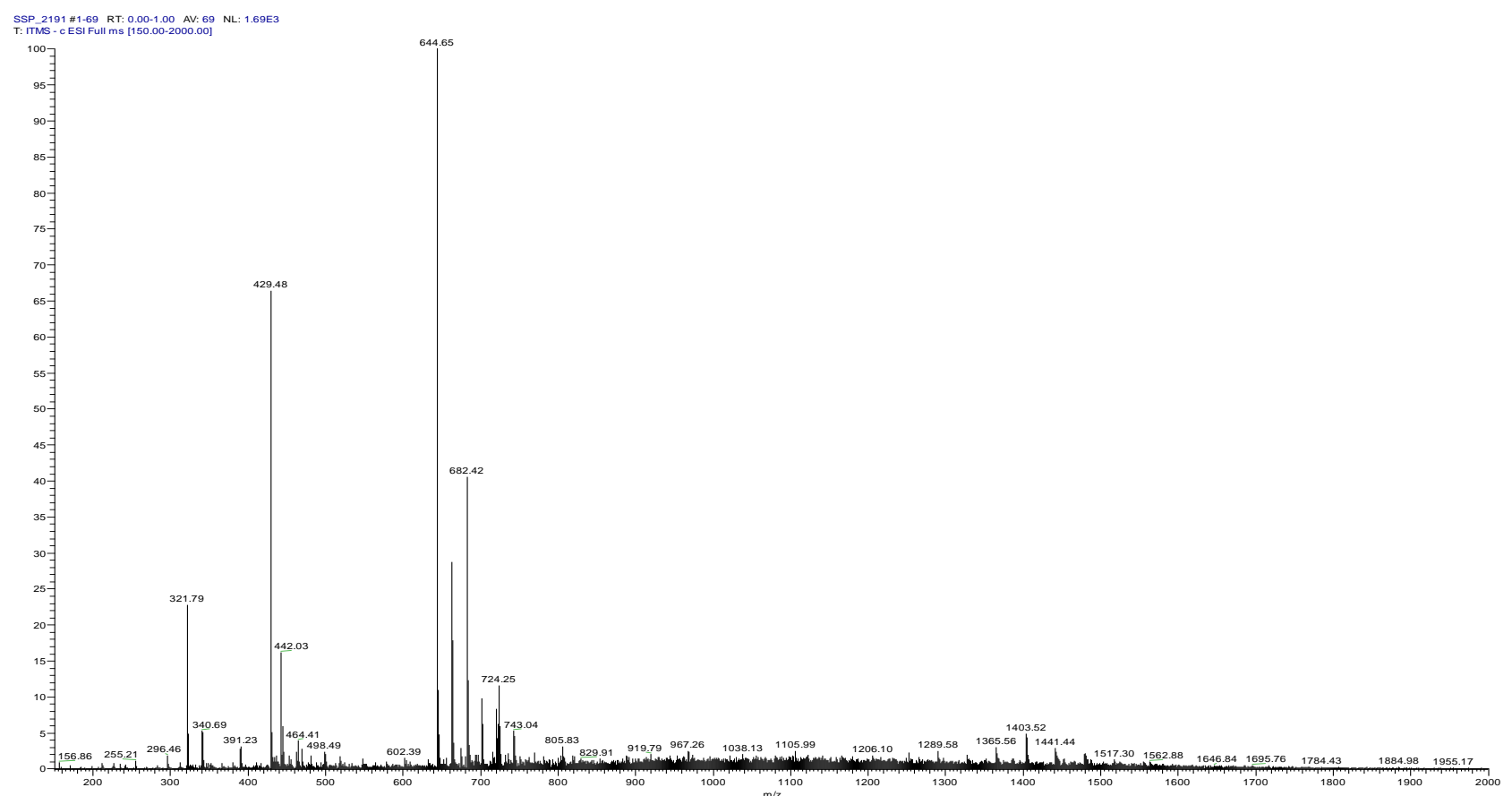

Figure S8. ESI- of $\mathrm{V}_{D A L}-2$. 


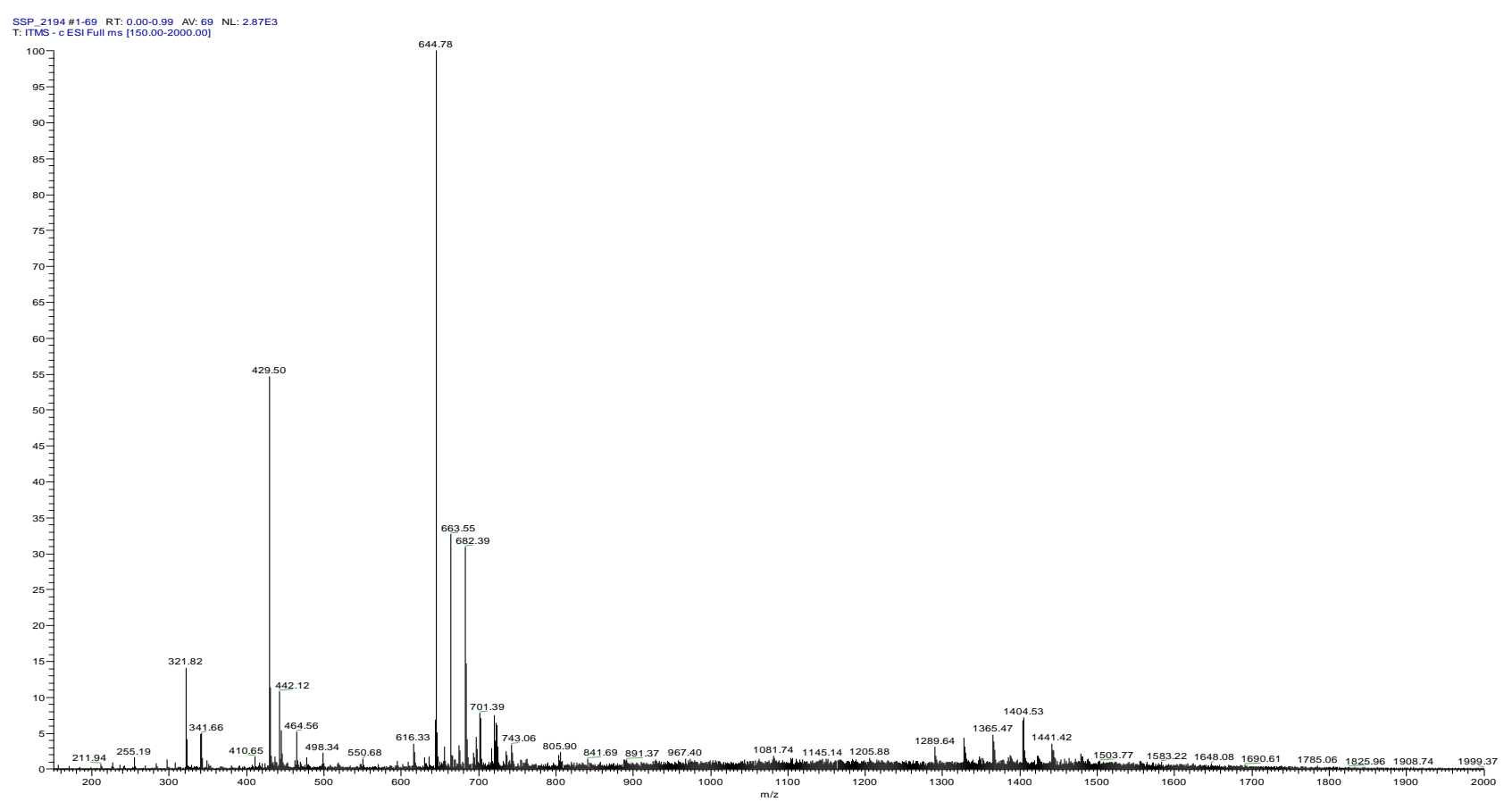

Figure S9. ESI- of VDAD-2.

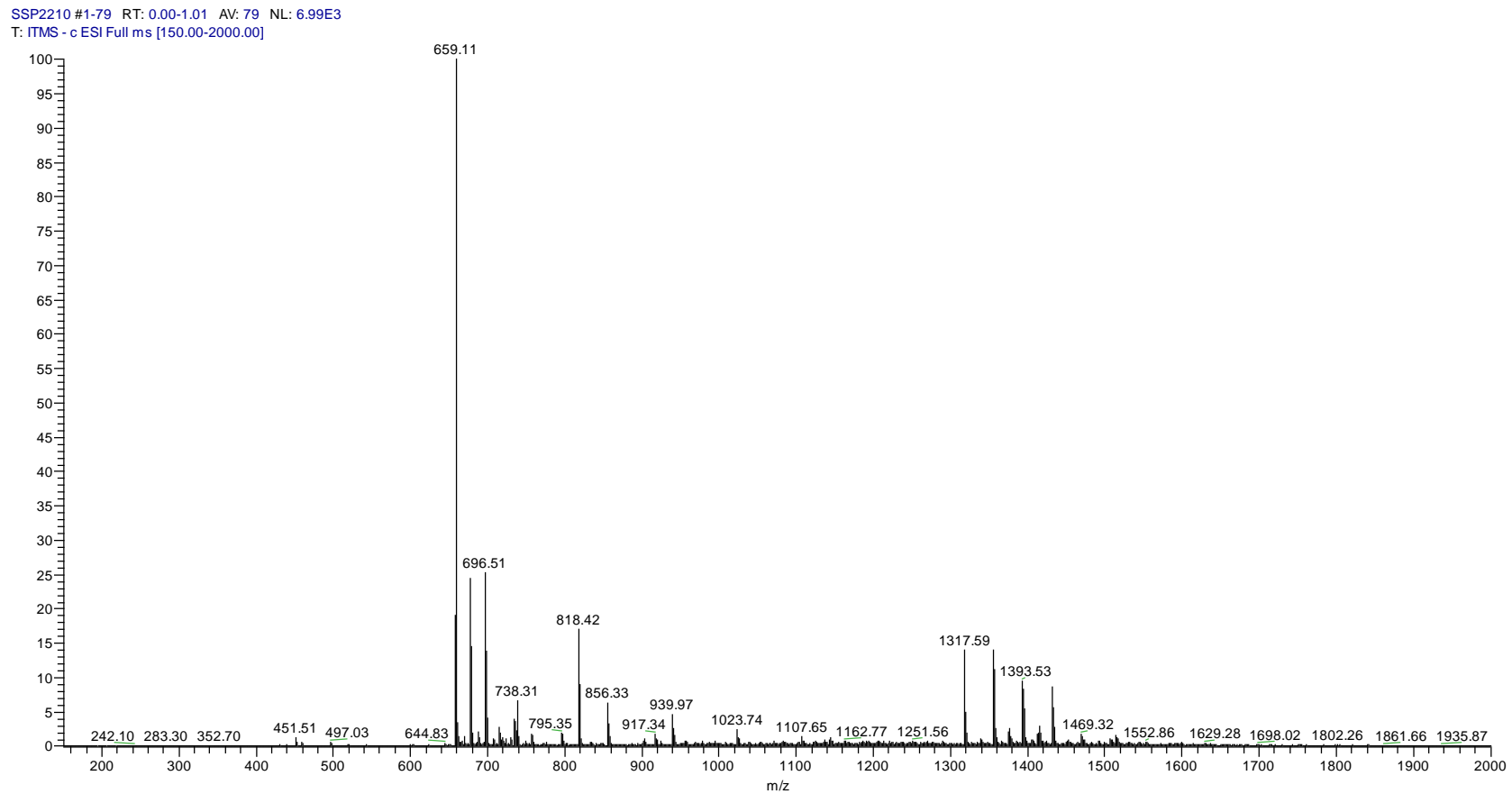

Figure S10. ESI- of $V_{L A D}-3$. 
2111_23 \#1-82 RT: 0.00-1.01 AV: 82 NL: 2.77E4

T: ITMS - c ESI Full ms [150.00-2000.00]
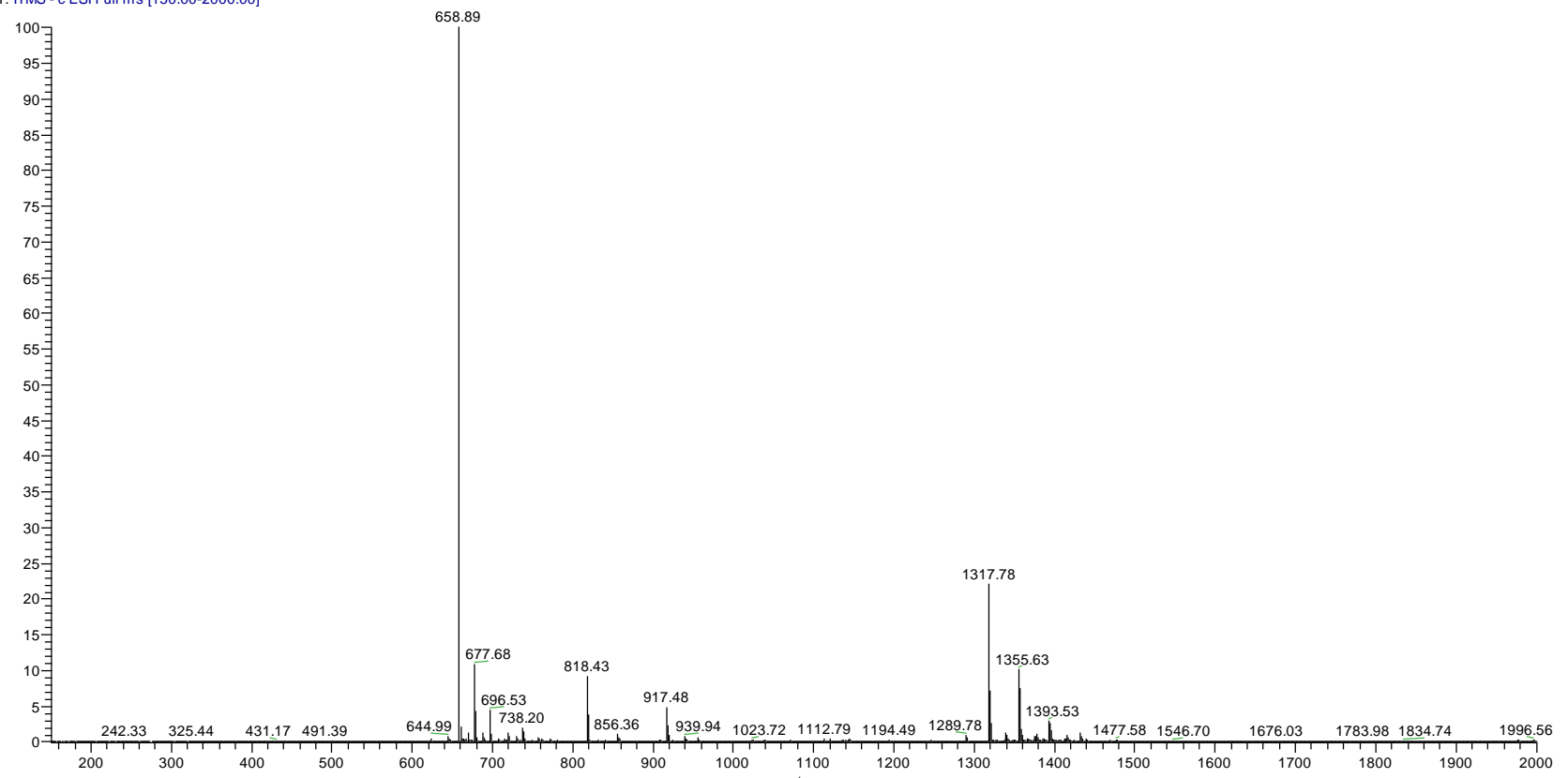

Figure S11. ESI- of $V_{D A L}-3$.

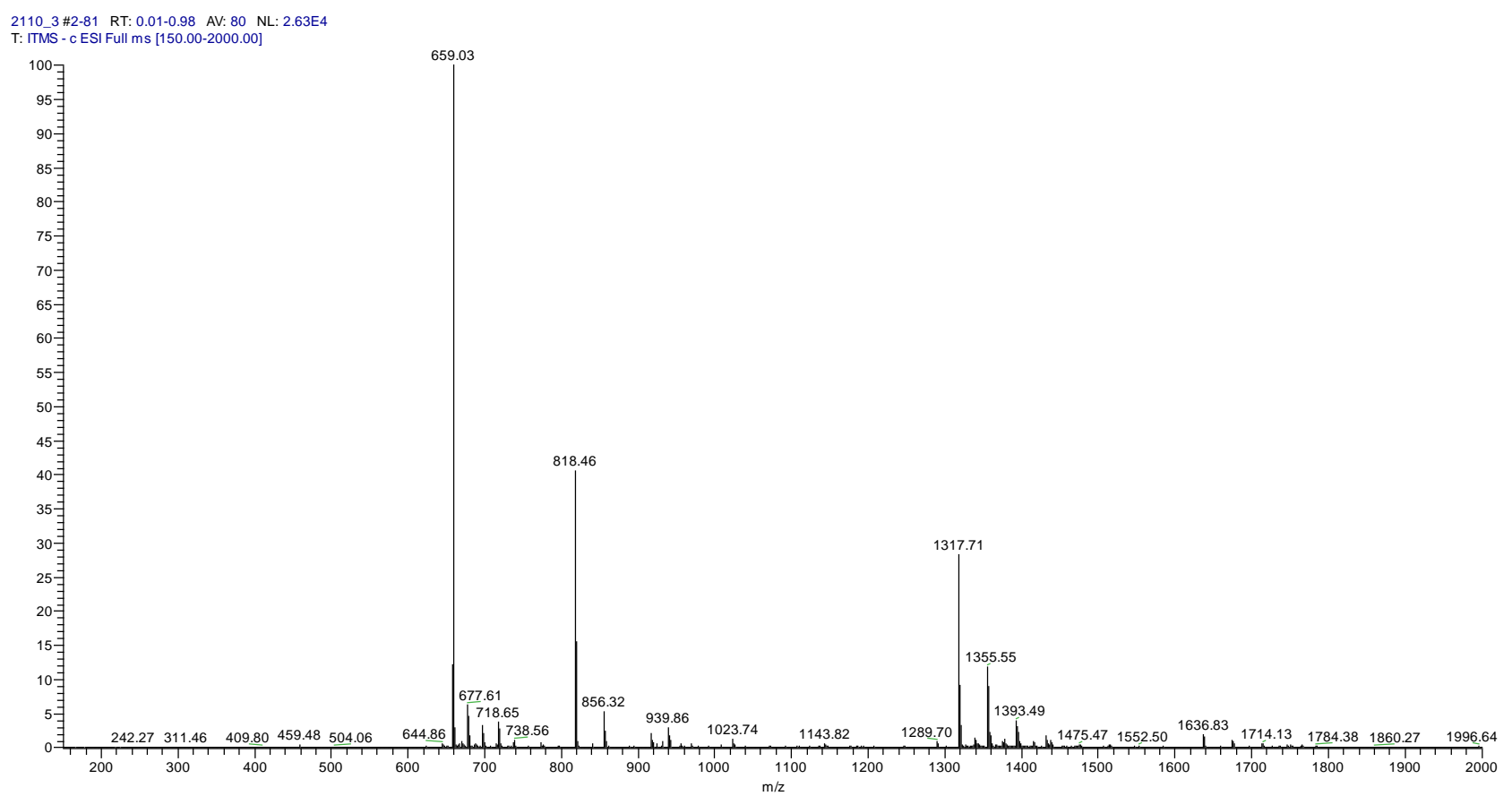

Figure S12. ESI- of VDAd-3. 


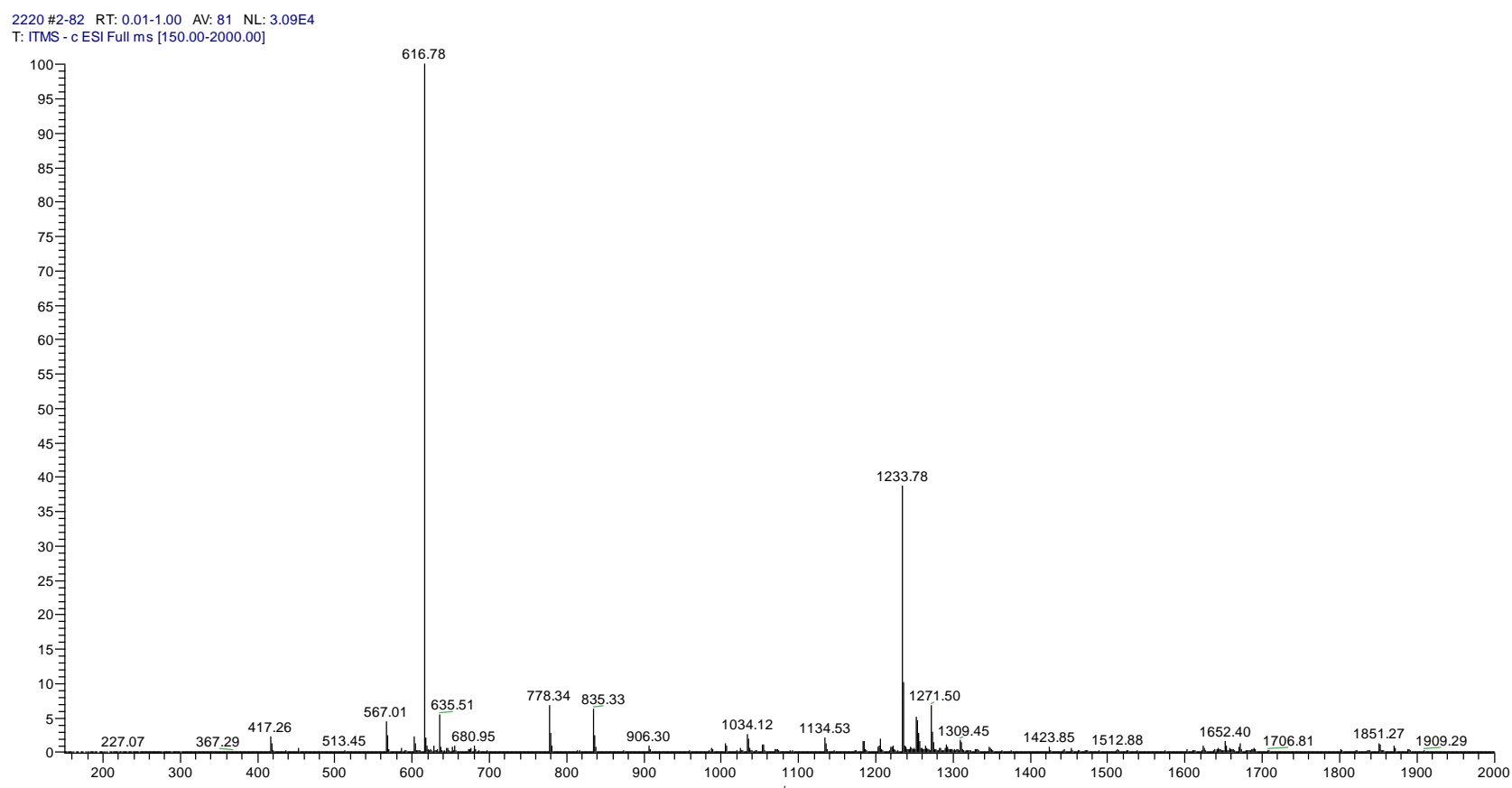

Figure S13. ESI- of $V_{D E V} V_{L} A_{L} G-P-T-P-G A_{L} V_{L} E V D$. 


\section{Analytical HPLC Traces}

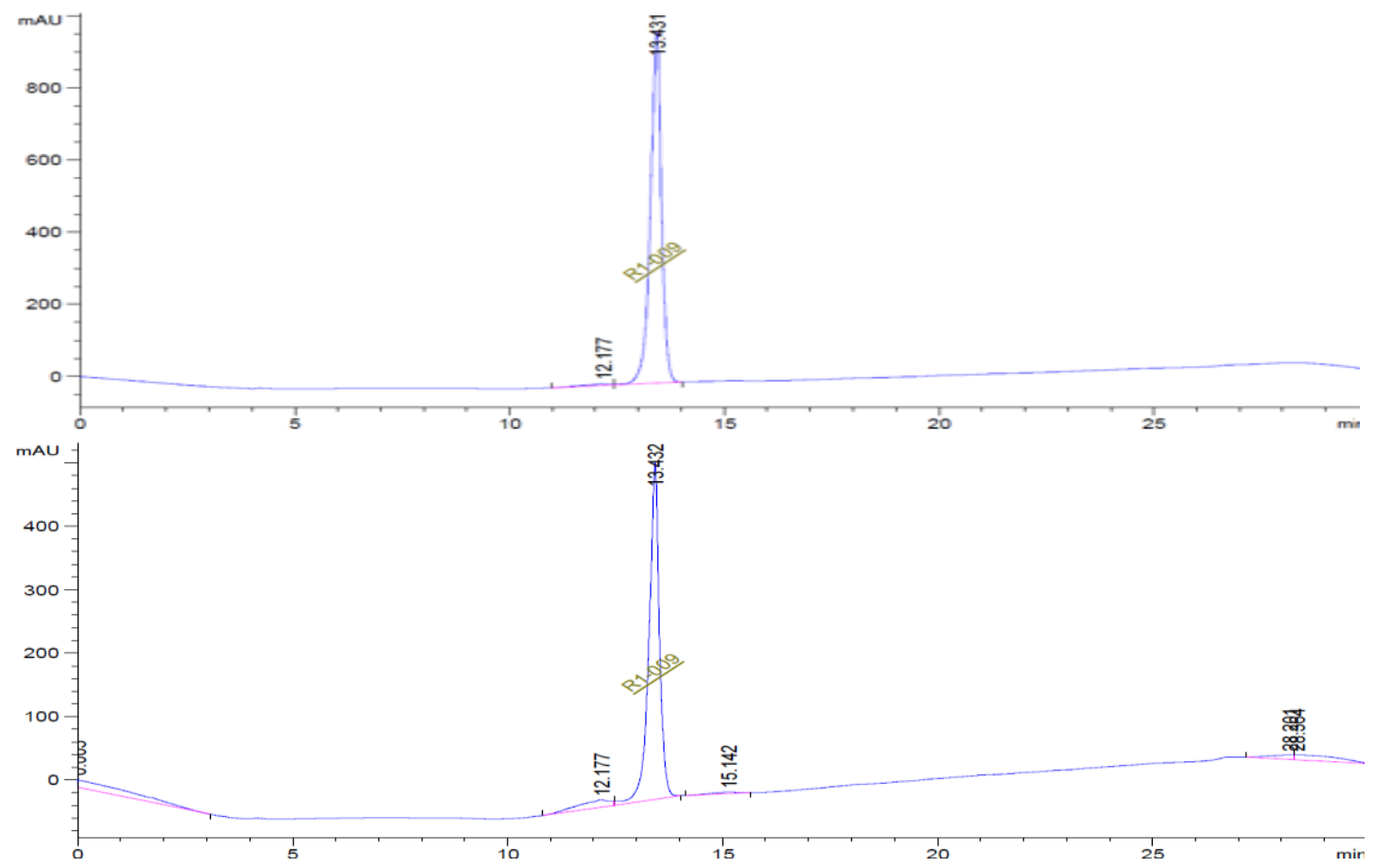

Figure S14. Analytical HPLC traces of $\mathbf{V}_{\mathbf{L}} \mathbf{A D}_{\mathbf{D}} \mathbf{- 0}$ monitoring $350 \mathrm{~nm}$ (top) and $260 \mathrm{~nm}$ (bottom).

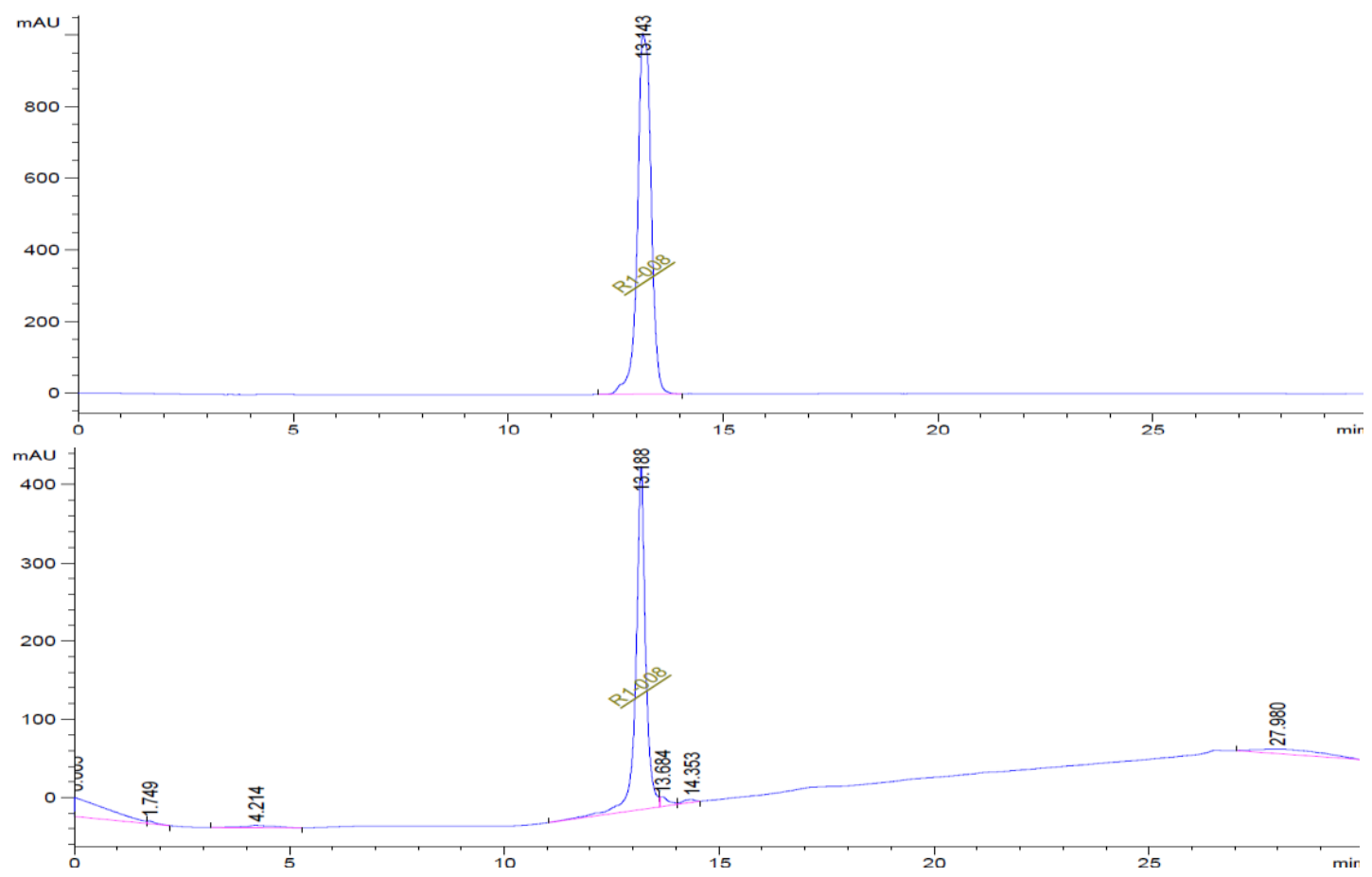

Figure S15. Analytical HPLC traces of $\mathbf{V}_{\mathbf{D}} \mathbf{A}_{\mathbf{L}} \mathbf{- 0}$ monitoring $350 \mathrm{~nm}$ (top) and $260 \mathrm{~nm}$ (bottom). 


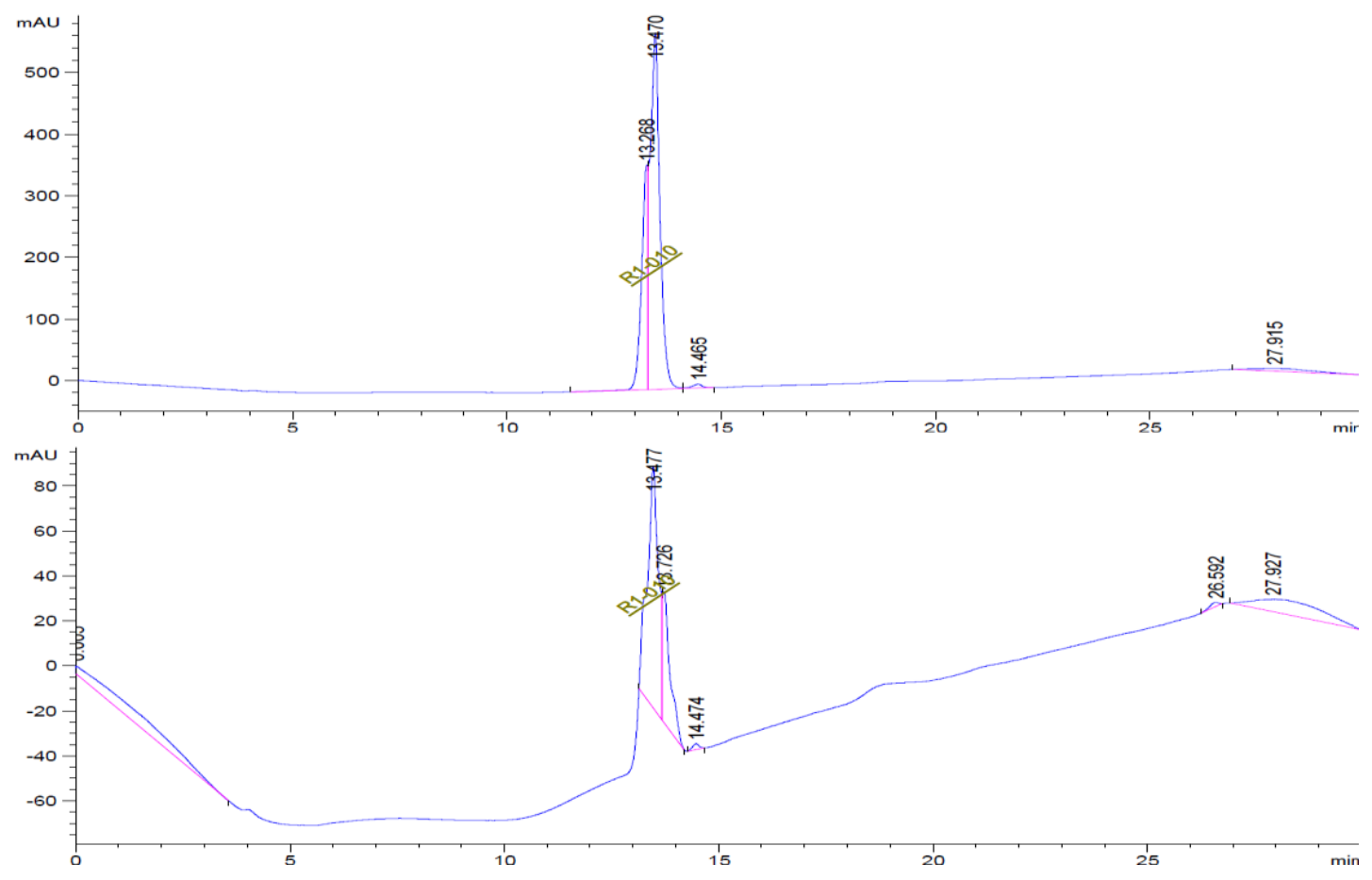

Figure S16. Analytical HPLC traces of $\mathbf{V}_{\mathbf{D}} \mathbf{A}_{\mathbf{D}} \mathbf{- 0}$ monitoring $350 \mathrm{~nm}$ (top) and $260 \mathrm{~nm}$ (bottom).

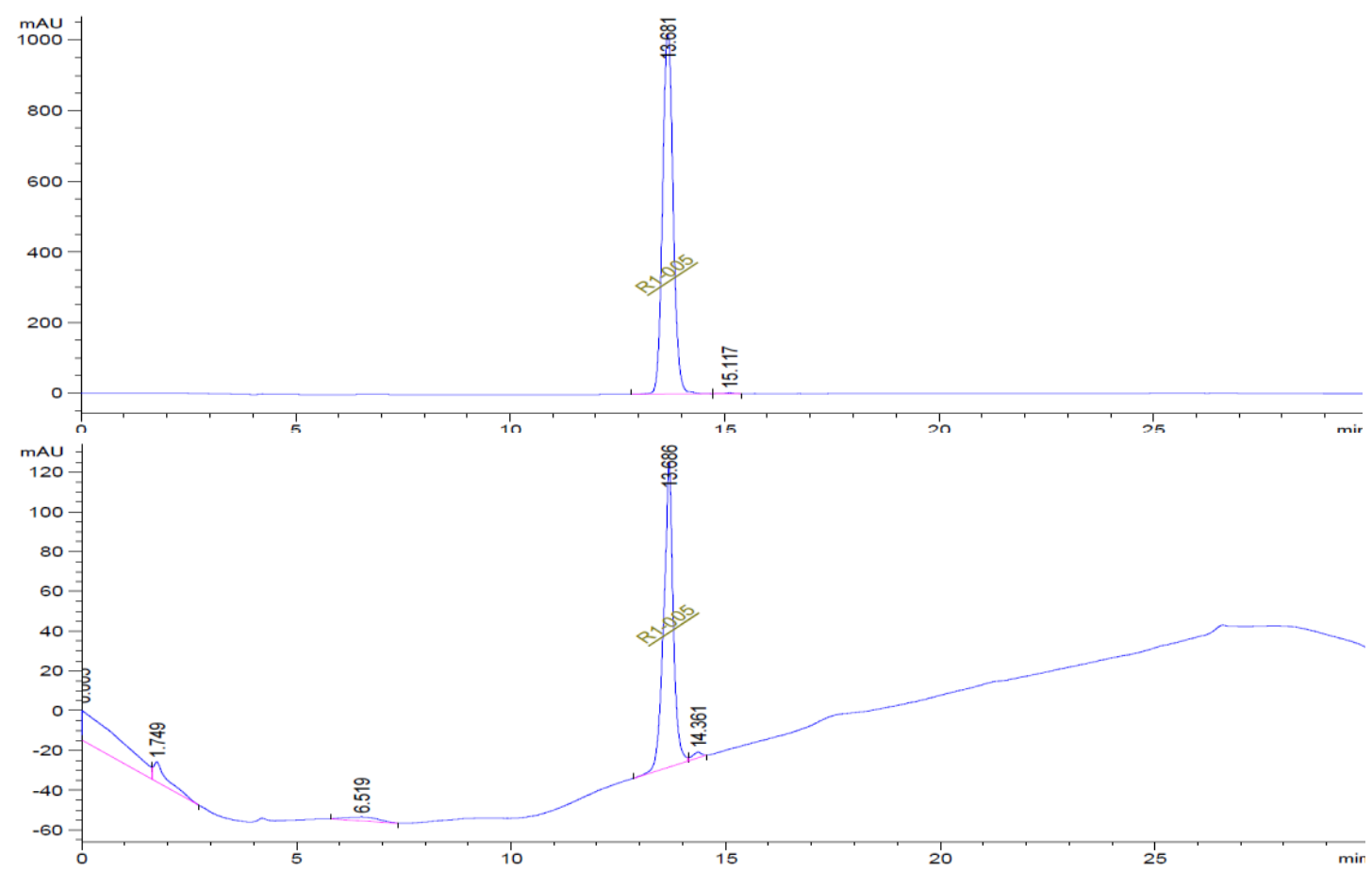

Figure S17. Analytical HPLC traces of $\mathbf{V}_{\mathbf{L}} \mathbf{A D}_{\mathbf{D}} \mathbf{- 1}$ monitoring $330 \mathrm{~nm}$ (top) and $260 \mathrm{~nm}$ (bottom). 


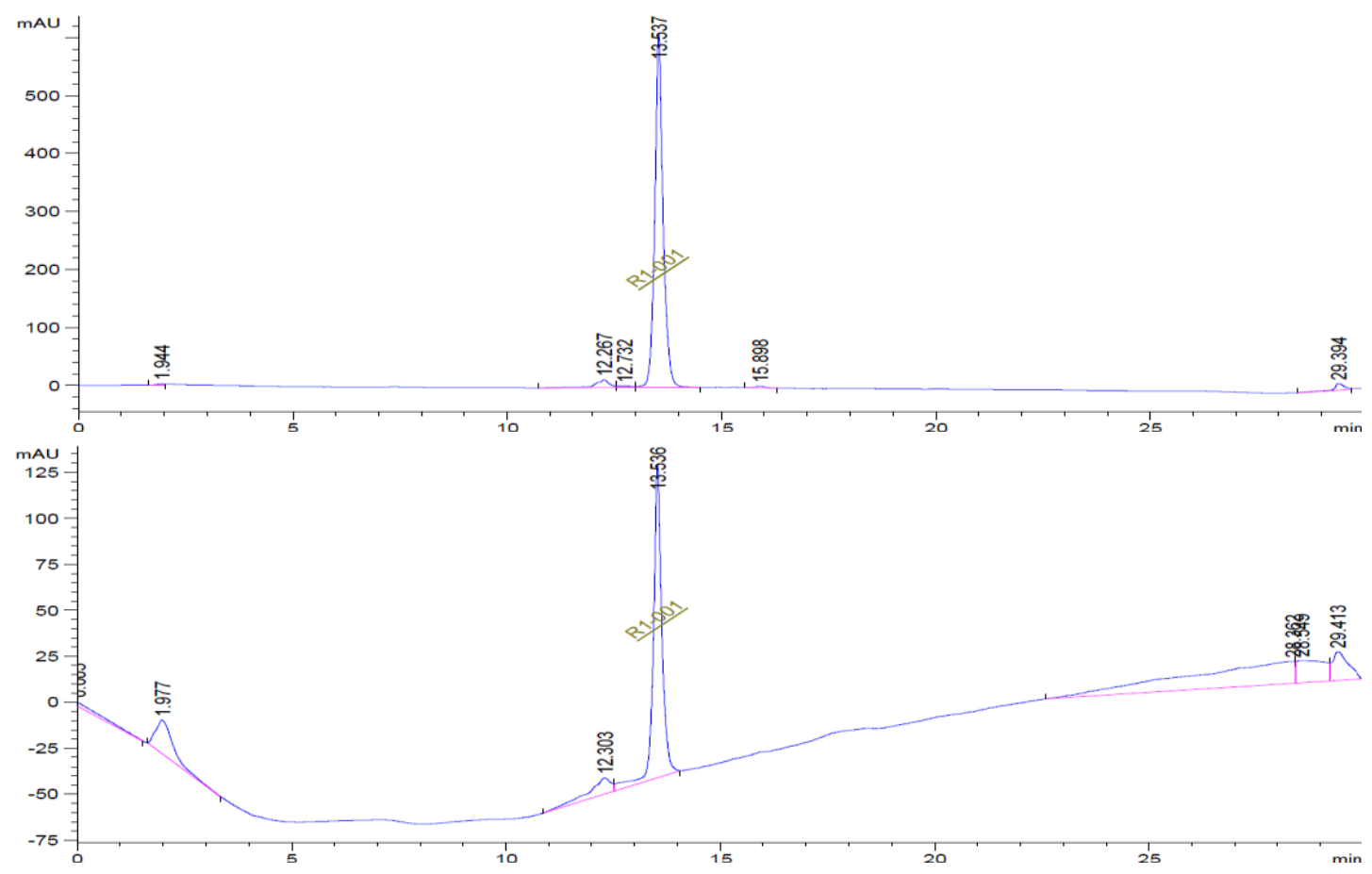

Figure S18. Analytical HPLC traces of VDAL-1 monitoring $330 \mathrm{~nm}$ (top) and $260 \mathrm{~nm}$ (bottom).

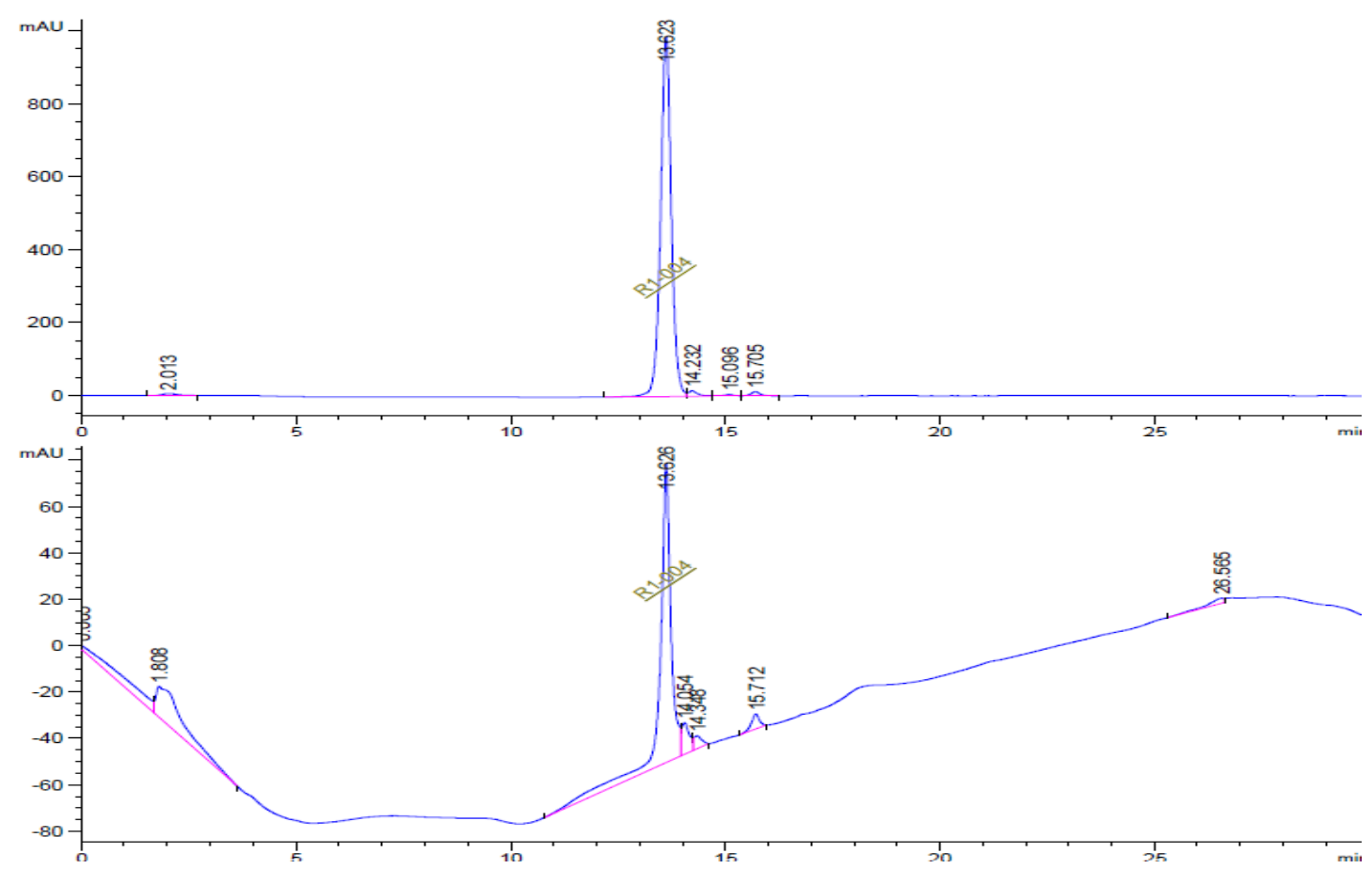

Figure S19. Analytical HPLC traces of VDAD-1 monitoring $330 \mathrm{~nm}$ (top) and $260 \mathrm{~nm}$ (bottom). 


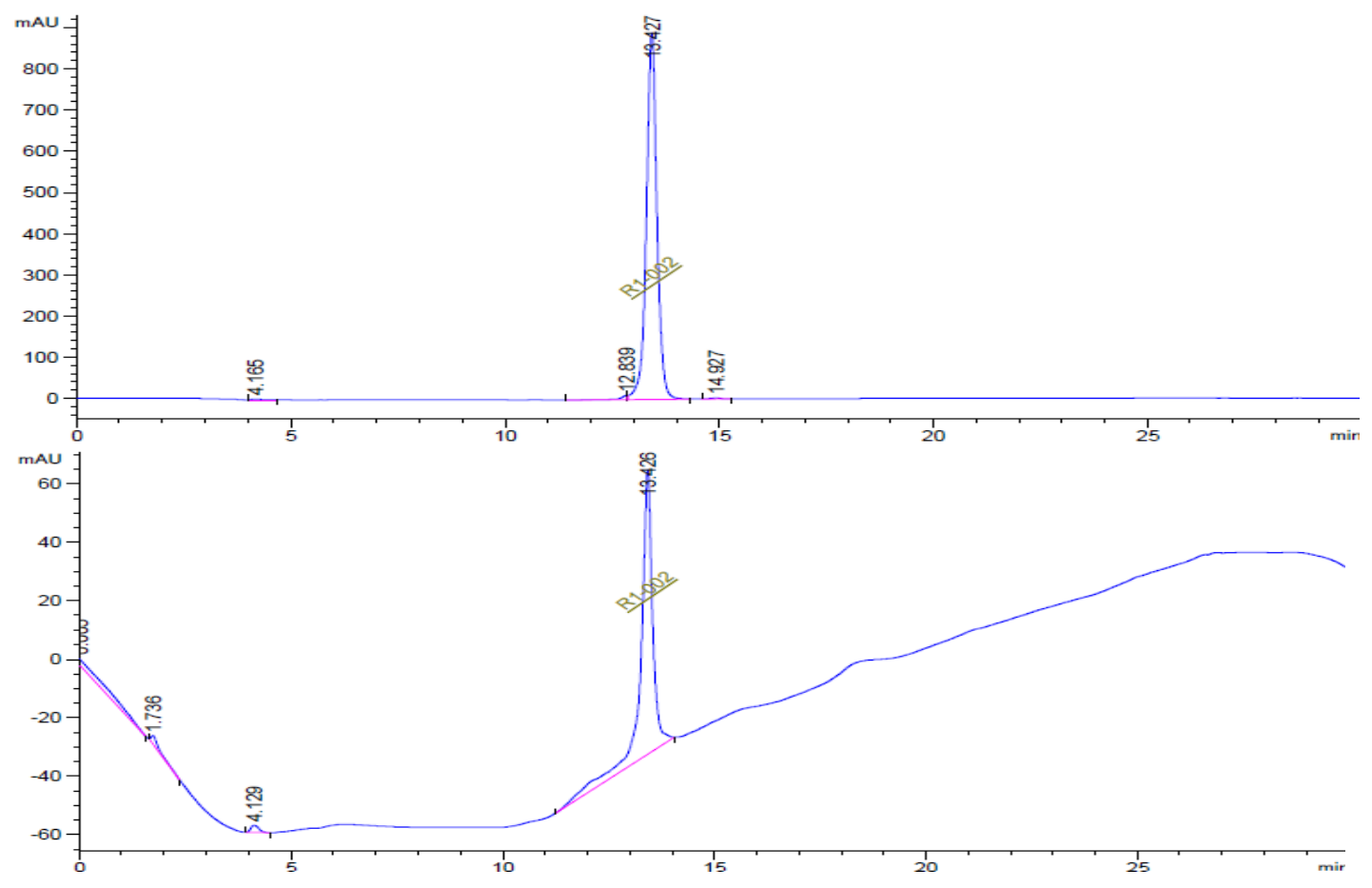

Figure S20. Analytical HPLC traces of VLAd-2 monitoring $330 \mathrm{~nm}$ (top) and $260 \mathrm{~nm}$ (bottom).

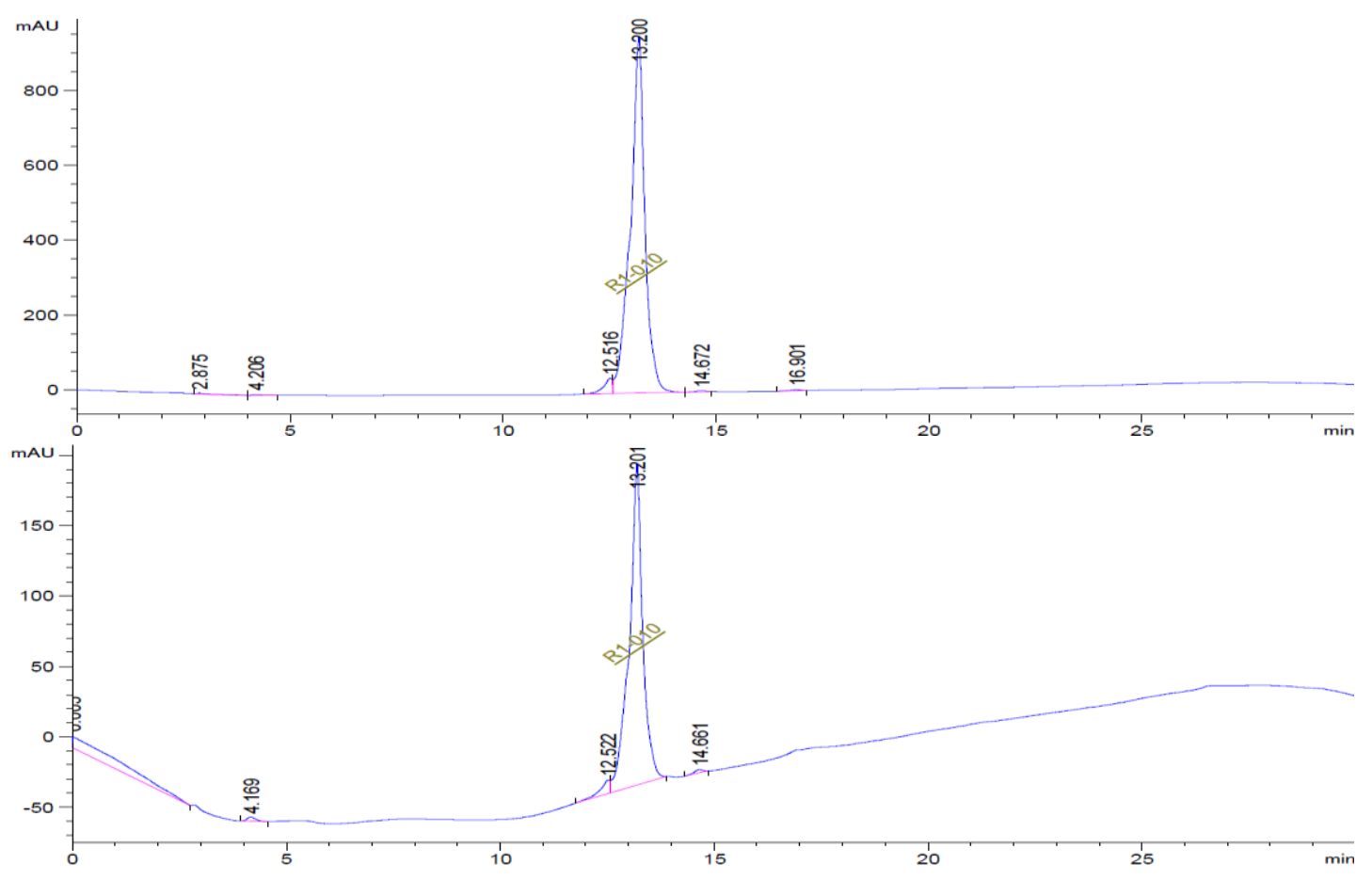

Figure S21. Analytical HPLC traces of VDAL-2 monitoring $330 \mathrm{~nm}$ (top) and $260 \mathrm{~nm}$ (bottom). 


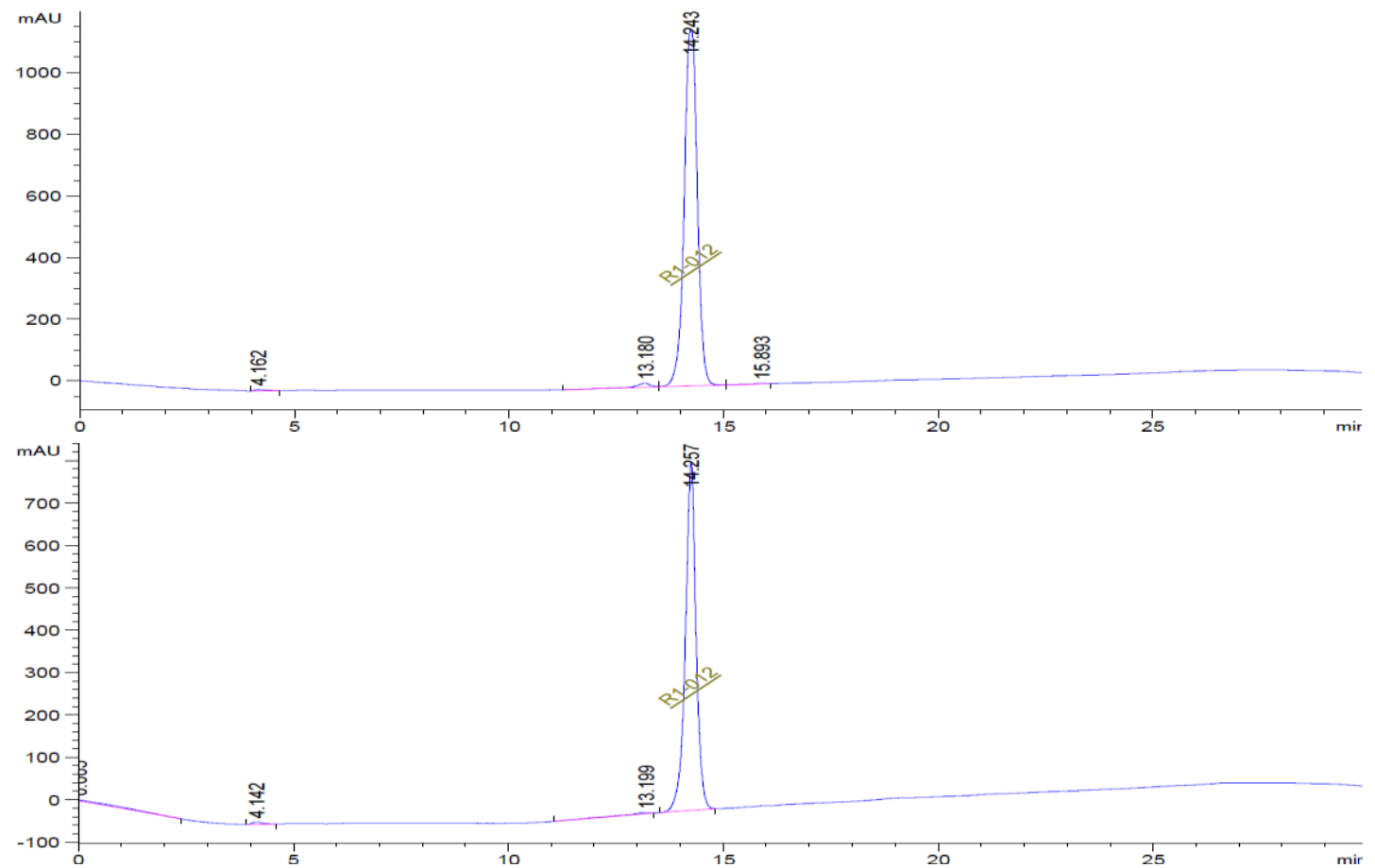

Figure S22. Analytical HPLC traces of $\mathbf{V}_{\mathbf{D}} \mathbf{A}_{\mathbf{D}} \mathbf{- 2}$ monitoring $330 \mathrm{~nm}$ (top) and $260 \mathrm{~nm}$ (bottom).

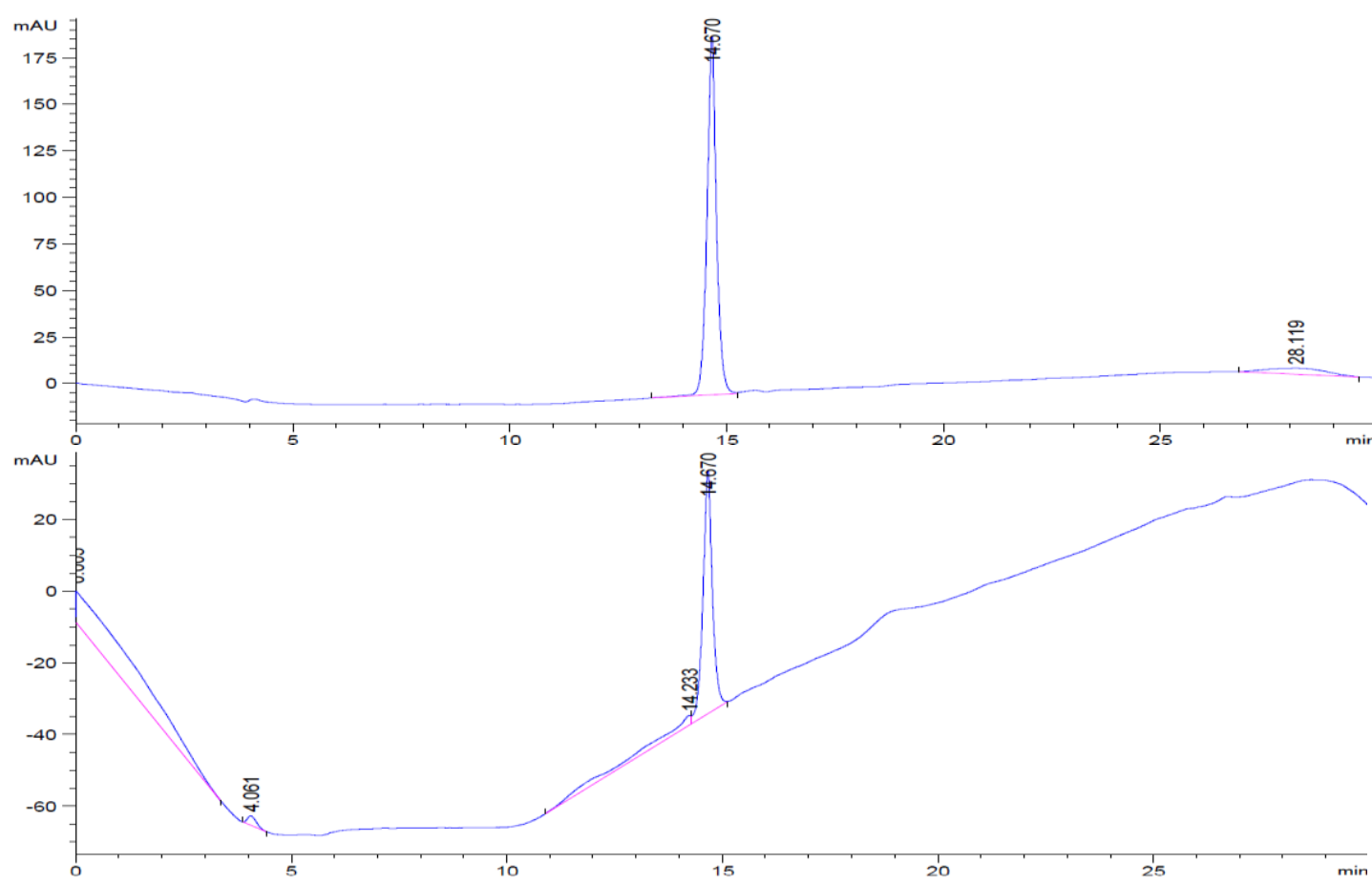

Figure S23. Analytical HPLC traces of VLAd-3 monitoring $330 \mathrm{~nm}$ (top) and $260 \mathrm{~nm}$ (bottom). 


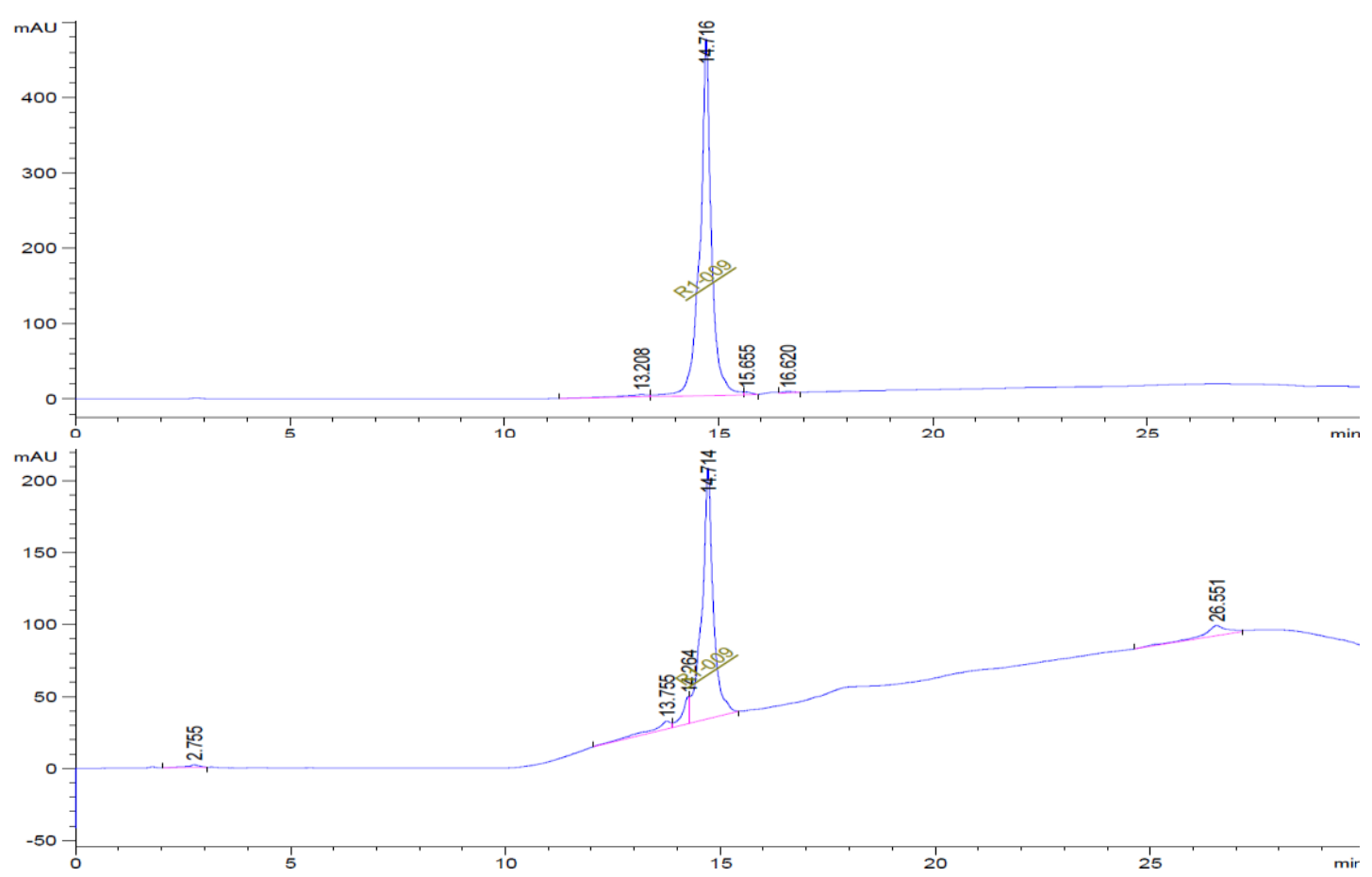

Figure S24. Analytical HPLC traces of VDAL-3 monitoring $330 \mathrm{~nm}$ (top) and $260 \mathrm{~nm}$ (bottom).

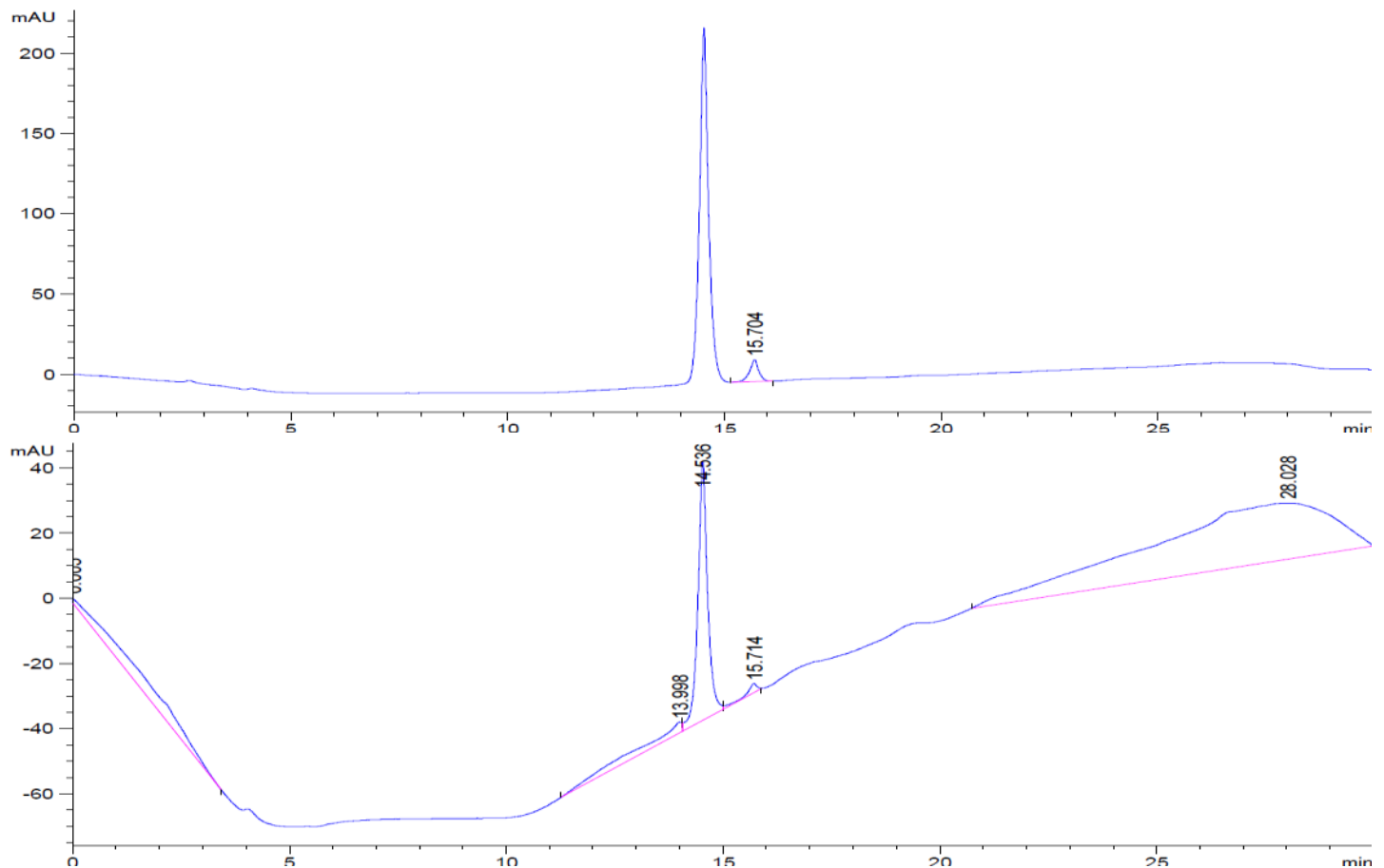

Figure S25. Analytical HPLC traces of VDAD-3 monitoring $330 \mathrm{~nm}$ (top) and $260 \mathrm{~nm}$ (bottom). 


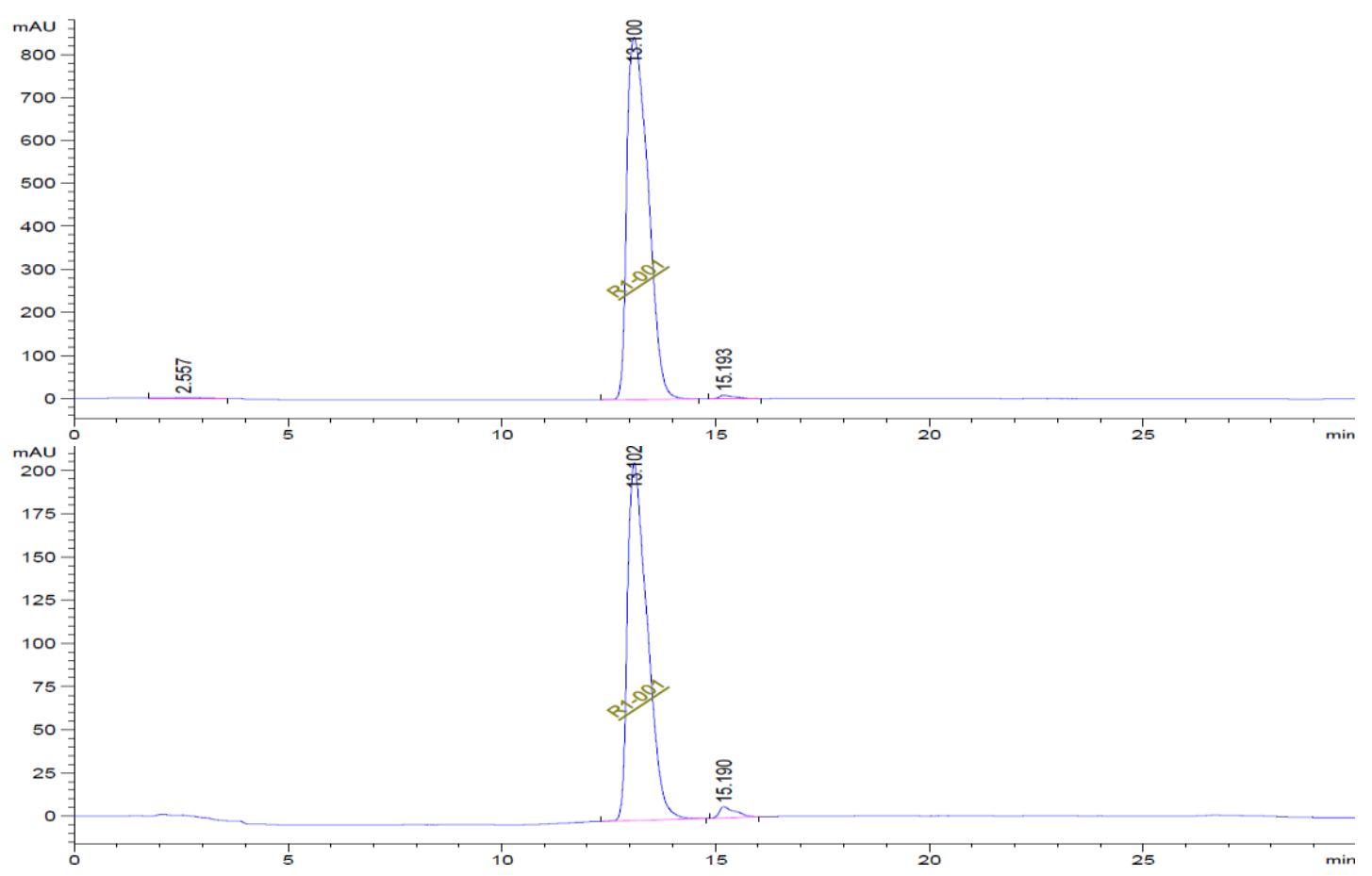

Figure S26. Analytical HPLC traces of $\mathbf{V}_{\mathbf{D}} \mathbf{E} \mathbf{V}_{\mathbf{L}} \mathbf{A}_{\mathbf{L}}$ G-P-T-P-GA $\mathbf{L} \mathbf{V}_{\mathbf{L}} \mathbf{E V} \mathbf{D}$ monitoring $350 \mathrm{~nm}$ (top) and $260 \mathrm{~nm}$ (bottom). 


\section{NMR Spectra}

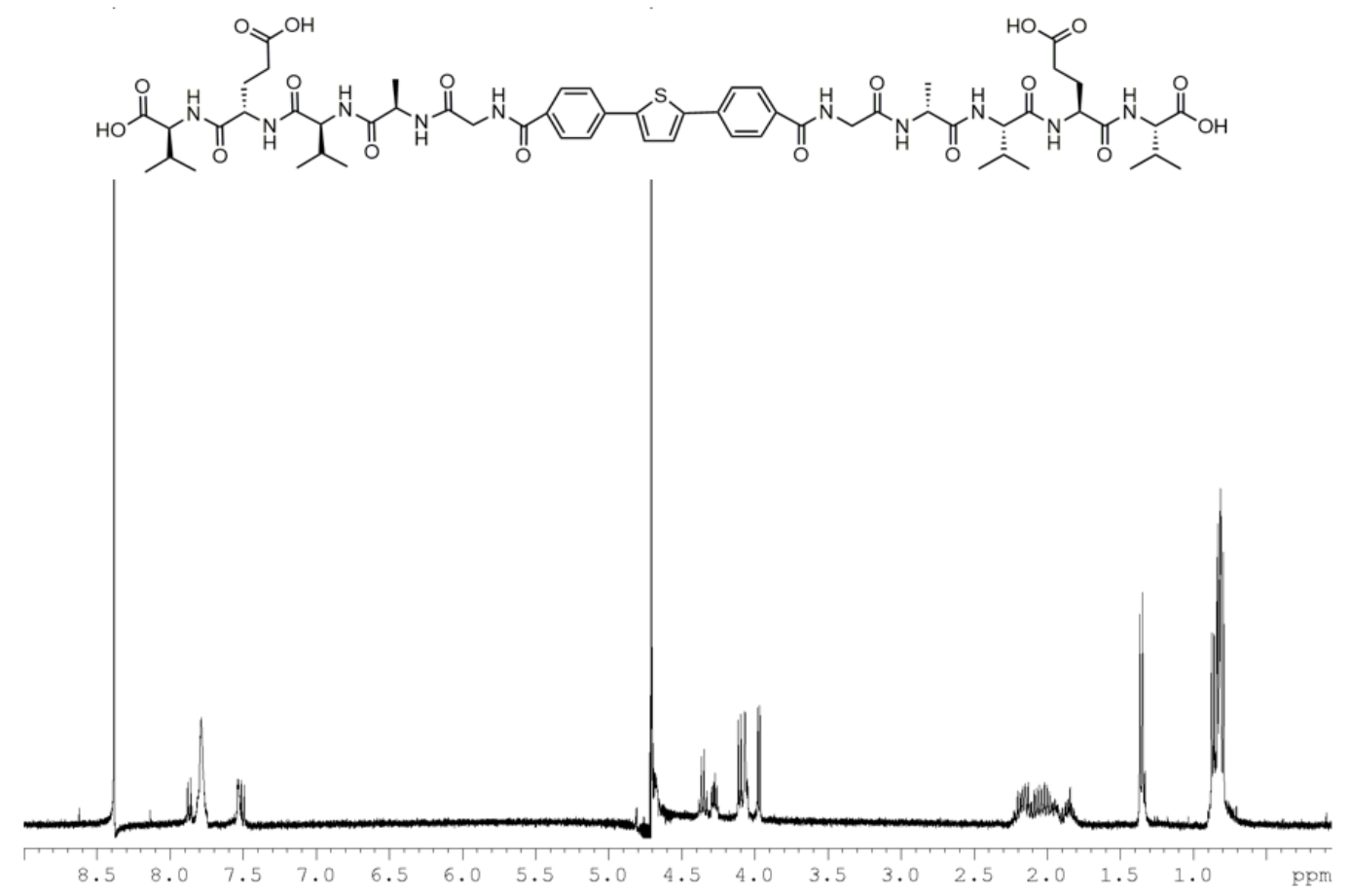

Figure S27. ${ }^{1} \mathrm{H}$ NMR $\left(\mathrm{D}_{2} \mathrm{O}, 400 \mathrm{MHz}\right)$ of VLAd-0.

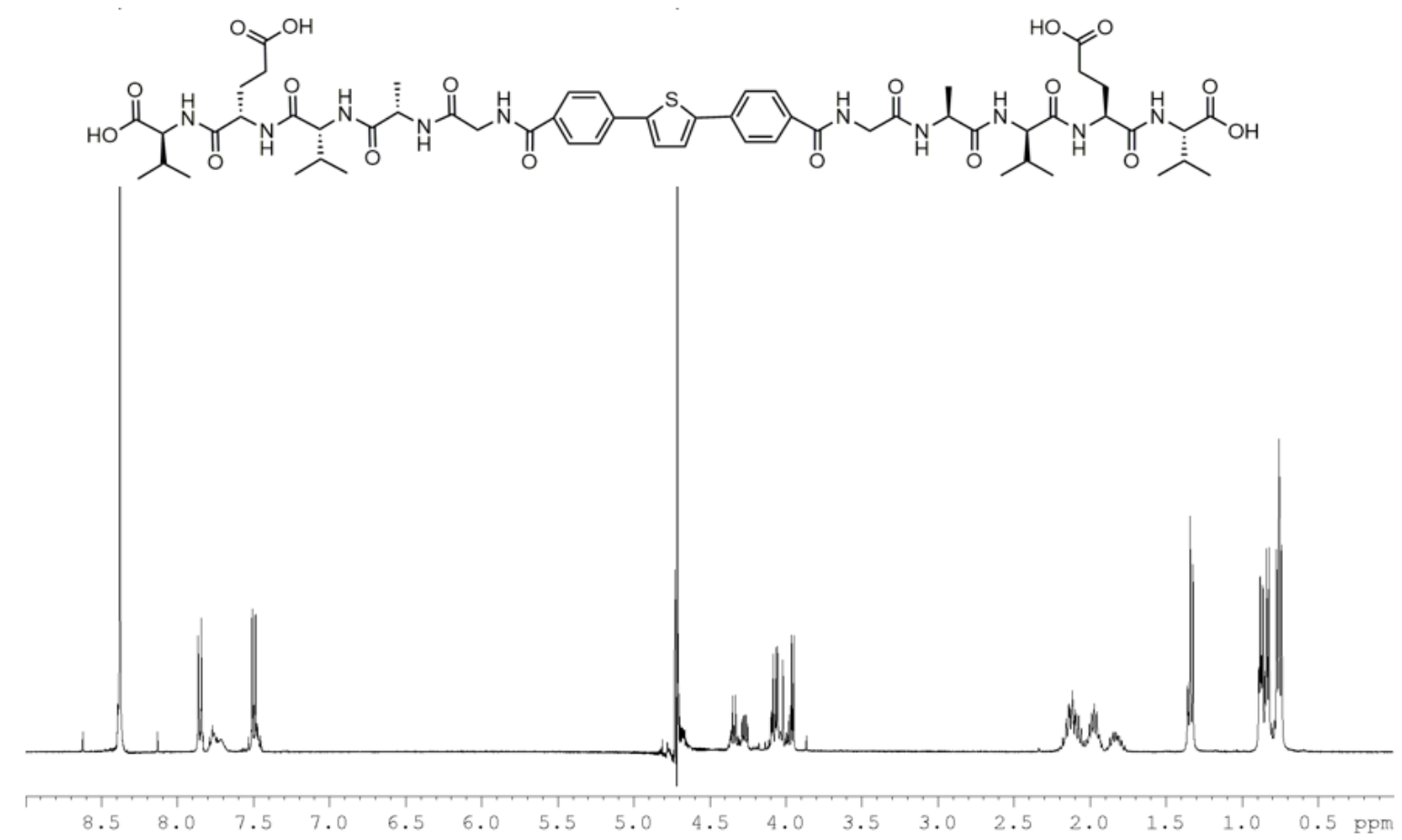

Figure S28. ${ }^{1} \mathrm{H}$ NMR $\left(\mathrm{D}_{2} \mathrm{O}, 400 \mathrm{MHz}\right)$ of $\mathbf{V}_{\mathbf{D}} \mathbf{A}_{\mathbf{L}}-\mathbf{0}$. 


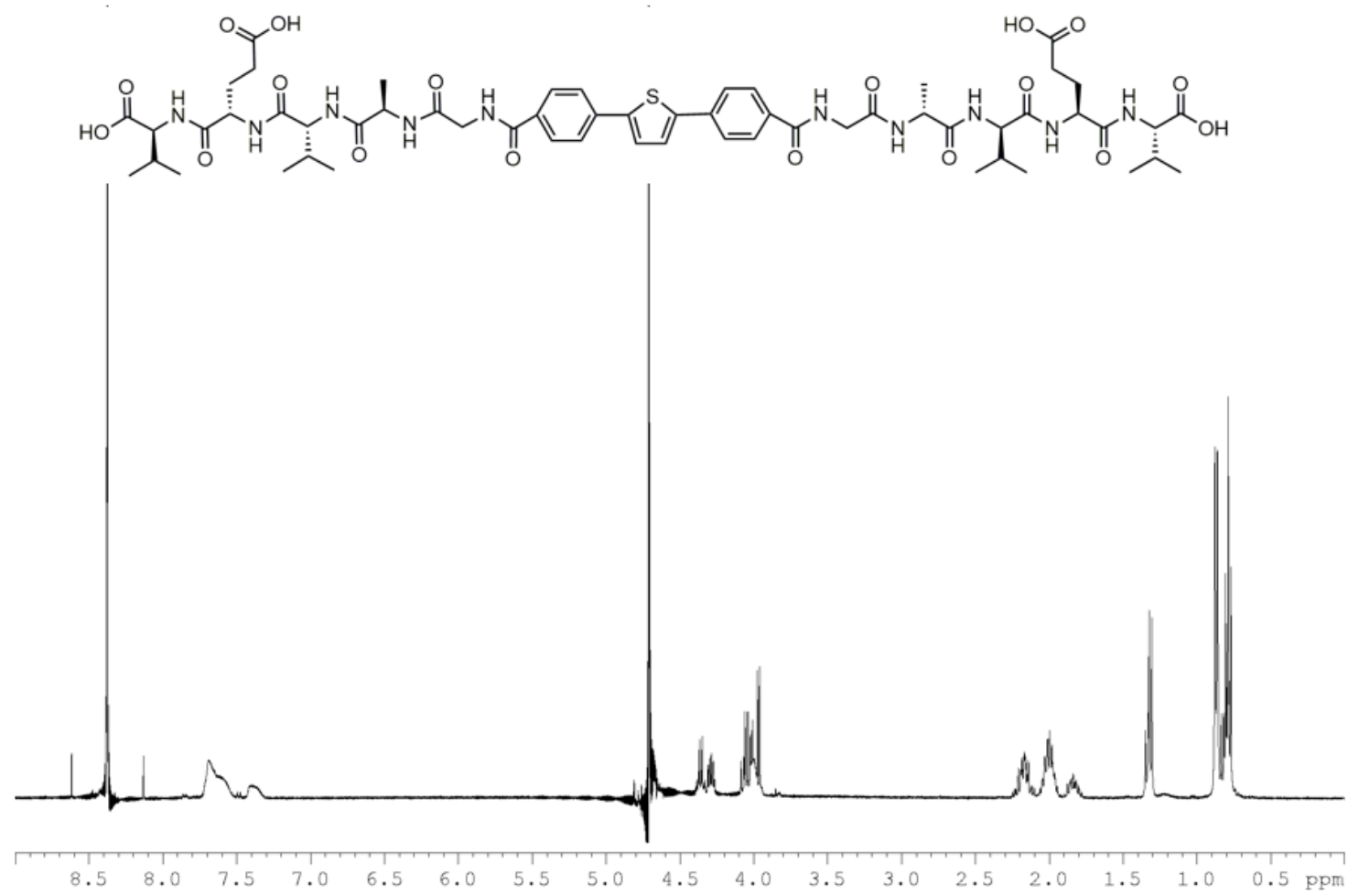

Figure S29. ${ }^{1} \mathrm{H}$ NMR $\left(\mathrm{D}_{2} \mathrm{O}, 400 \mathrm{MHz}\right)$ of $\mathbf{V}_{\mathbf{D}} \mathbf{A D}-\mathbf{0}$.

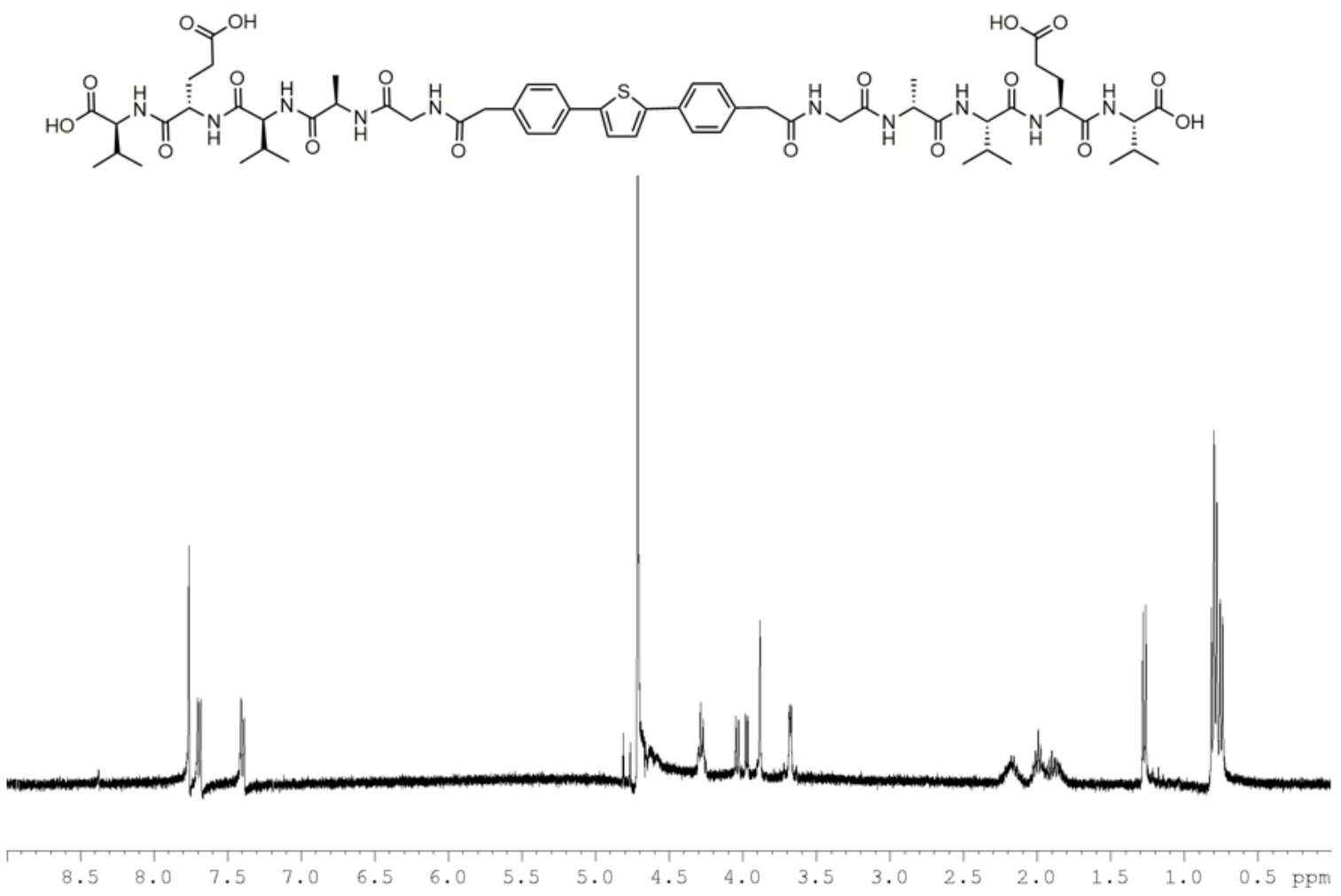

Figure S30. ${ }^{1} \mathrm{H}$ NMR $\left(\mathrm{D}_{2} \mathrm{O}, 400 \mathrm{MHz}\right)$ of $\mathbf{V}_{\mathbf{L A D}} \mathbf{A}-1$. 


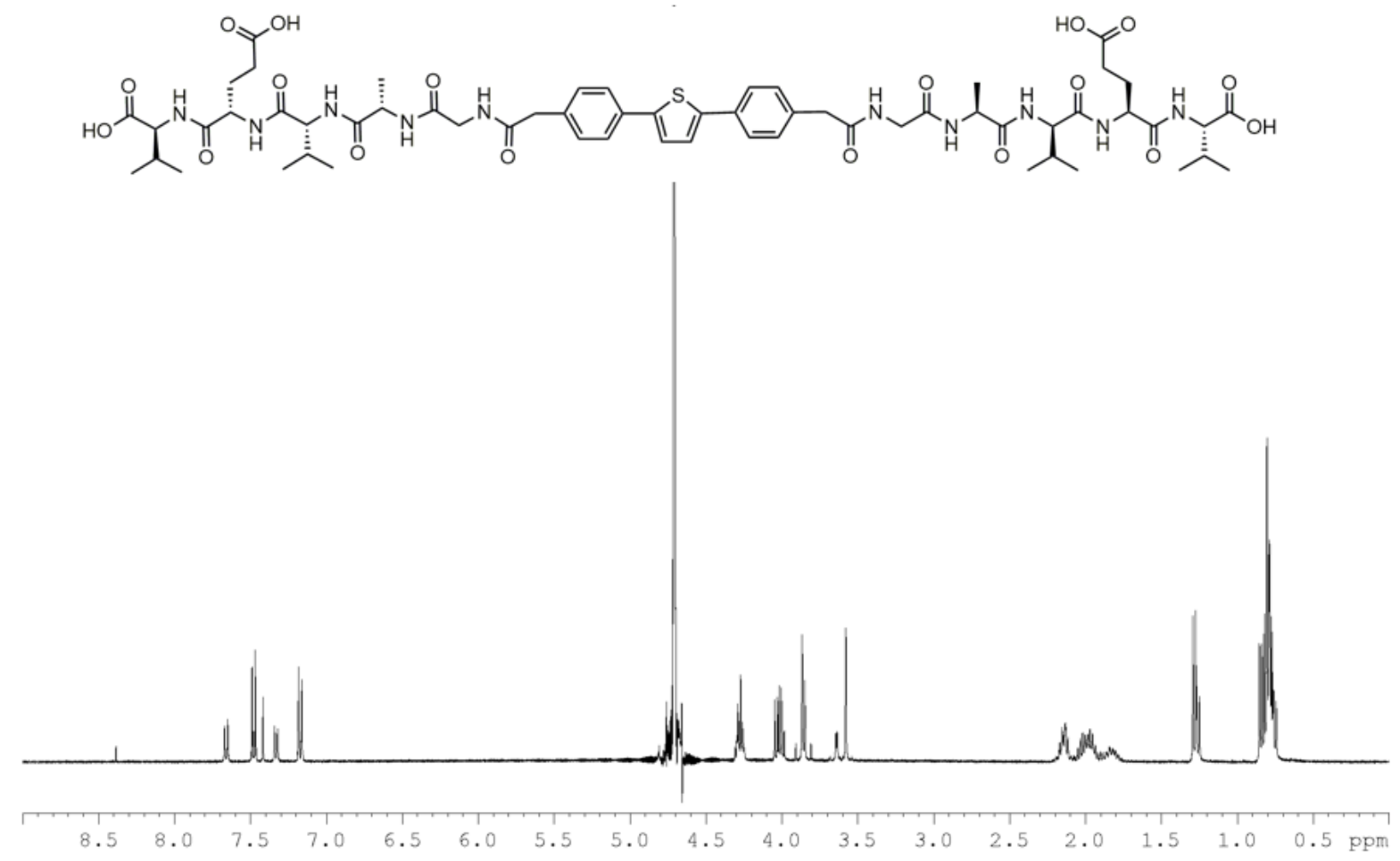

Figure S31. ${ }^{1} \mathrm{H}$ NMR $\left(\mathrm{D}_{2} \mathrm{O}, 400 \mathrm{MHz}\right)$ of $\mathbf{V}_{\mathbf{D}} \mathbf{A}_{\mathbf{L}} \mathbf{- 1}$.

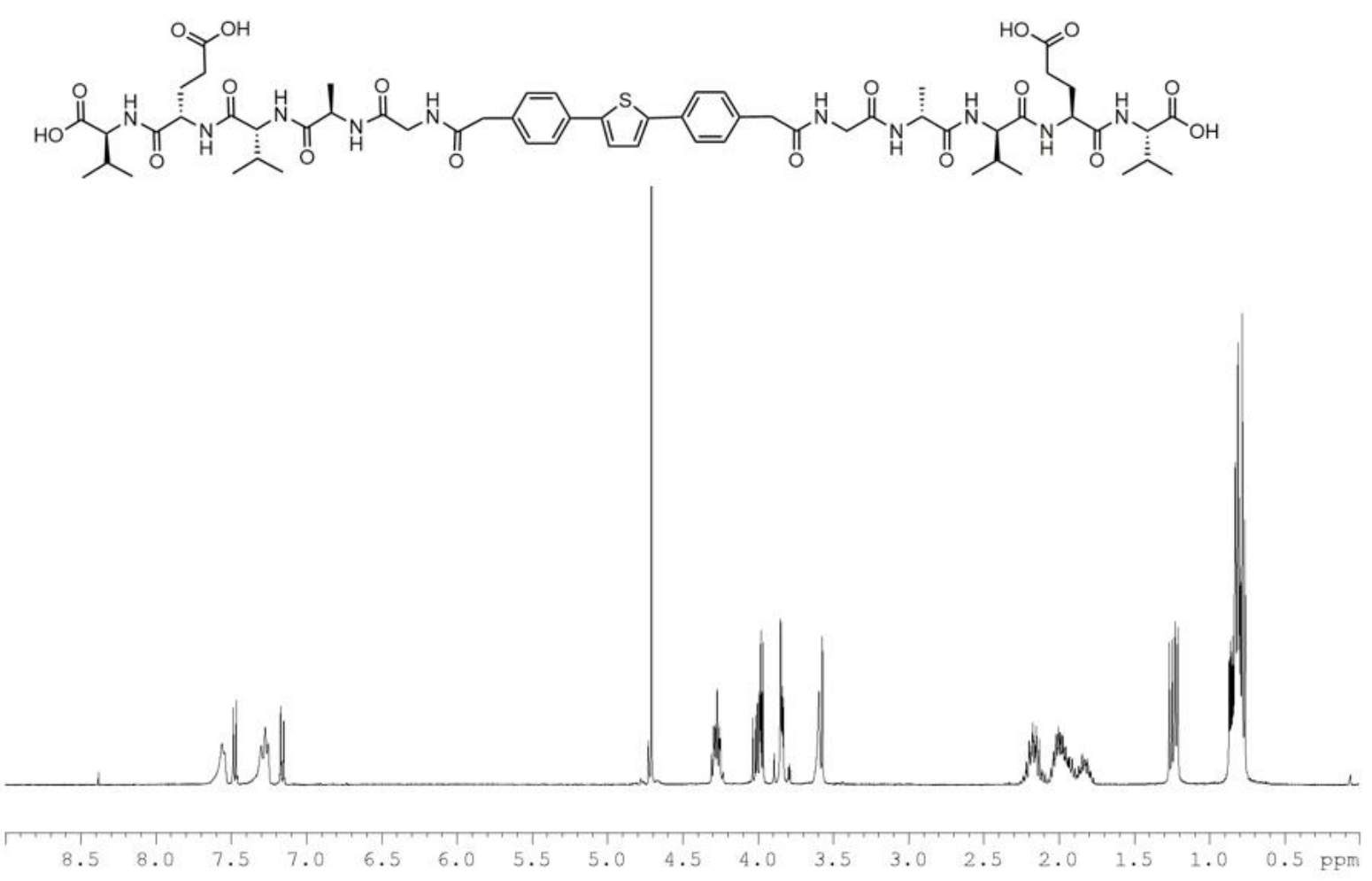

Figure S32. ${ }^{1} \mathrm{H}$ NMR $\left(\mathrm{D}_{2} \mathrm{O}, 400 \mathrm{MHz}\right)$ of $\mathbf{V}_{\mathbf{D}} \mathbf{A D}_{\mathbf{D}} \mathbf{- 1}$. 


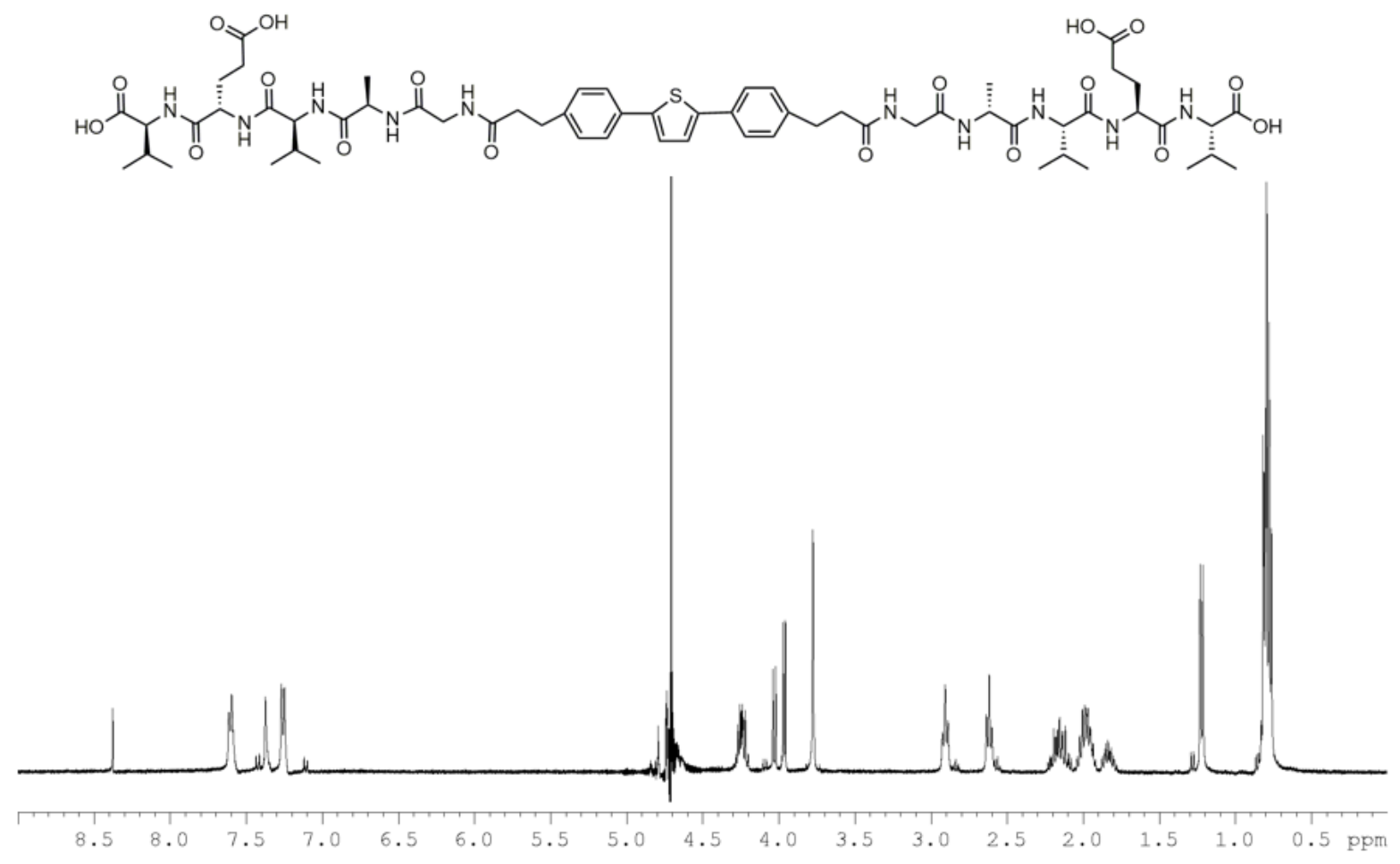

Figure S33. ${ }^{1} \mathrm{H}$ NMR $\left(\mathrm{D}_{2} \mathrm{O}, 400 \mathrm{MHz}\right)$ of VLAd-2.
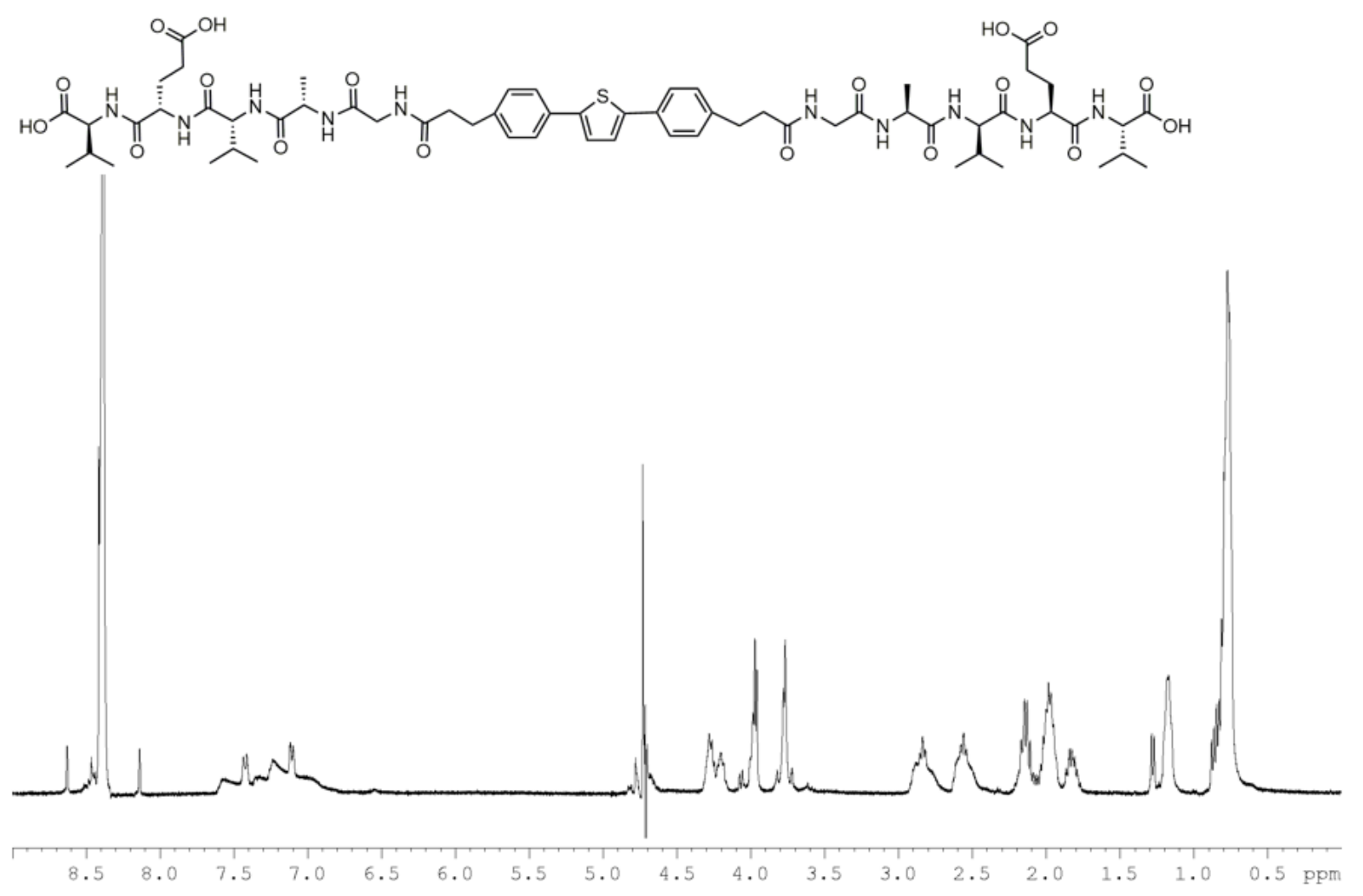

Figure S34. ${ }^{1} \mathrm{H}$ NMR $\left(\mathrm{D}_{2} \mathrm{O}, 400 \mathrm{MHz}\right)$ of $\mathbf{V}_{\mathbf{D}} \mathbf{A}_{\mathbf{L}}-\mathbf{2}$. 

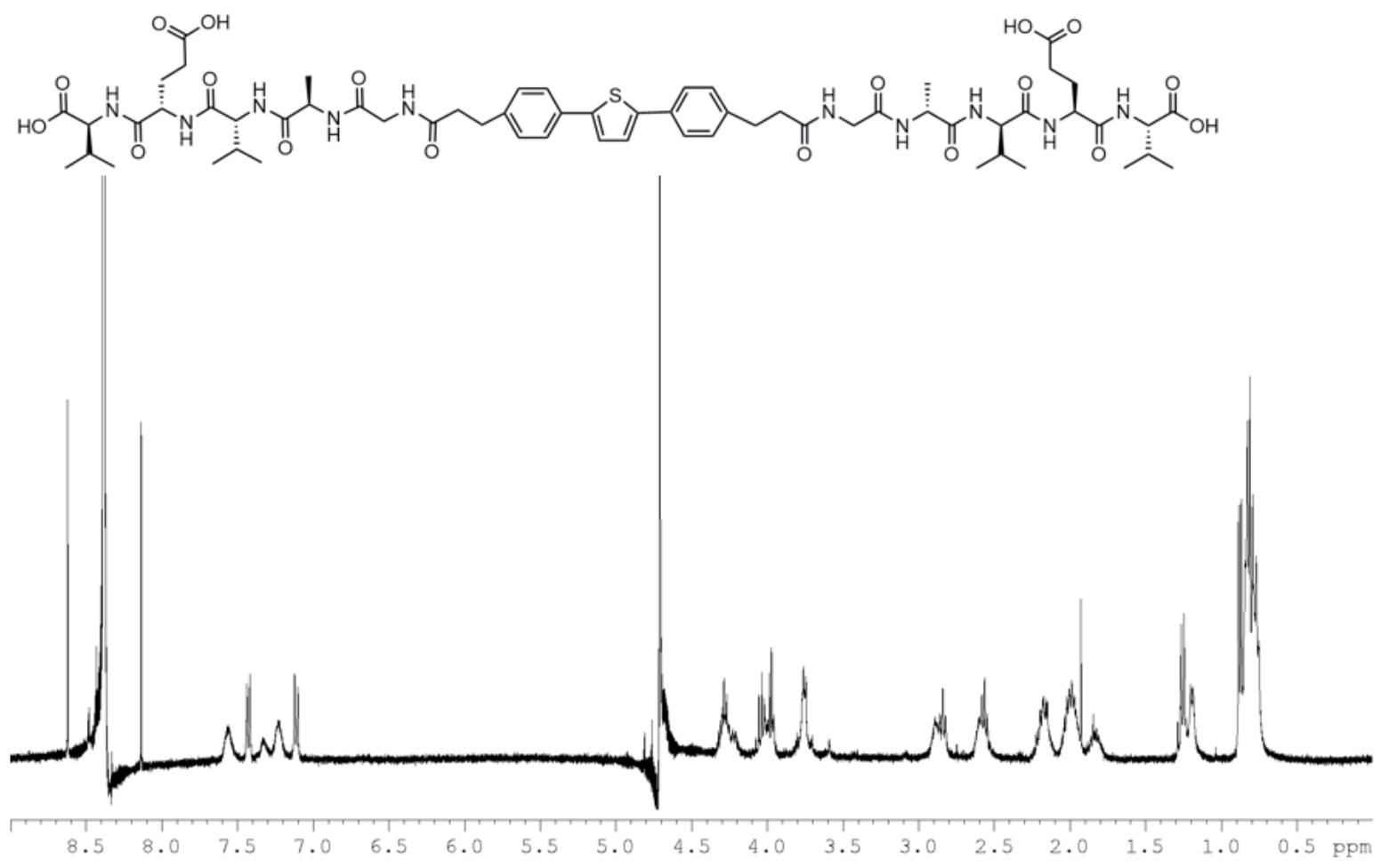

Figure S35. ${ }^{1} \mathrm{H}$ NMR $\left(\mathrm{D}_{2} \mathrm{O}, 400 \mathrm{MHz}\right)$ of $\mathbf{V}_{\mathbf{D}} \mathbf{A}_{\mathbf{D}}-\mathbf{2}$.
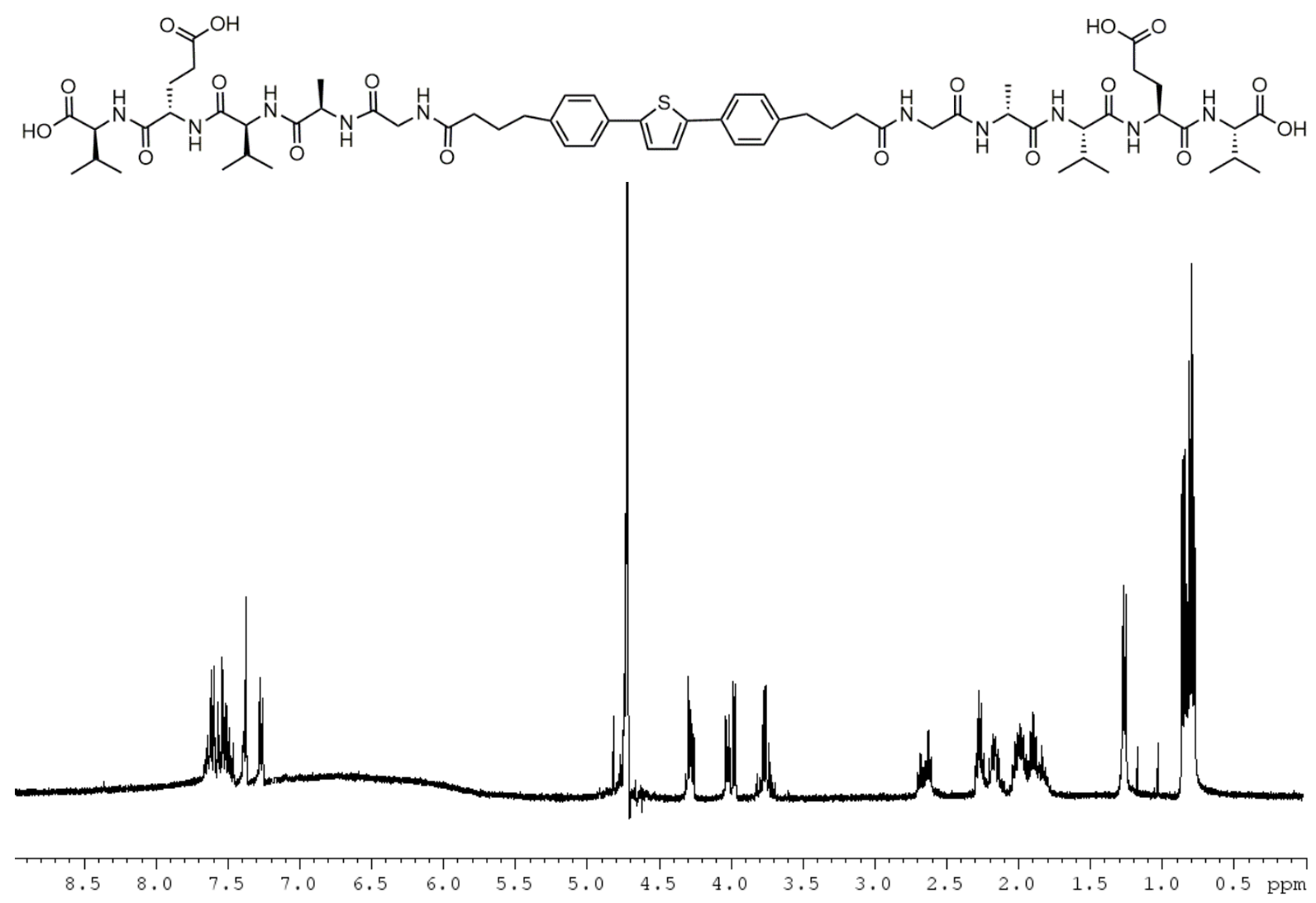

Figure S36. ${ }^{1} \mathrm{H}$ NMR $\left(\mathrm{D}_{2} \mathrm{O}, 400 \mathrm{MHz}\right)$ of $\mathbf{V}_{\mathbf{L A d}} \mathbf{A}$. 


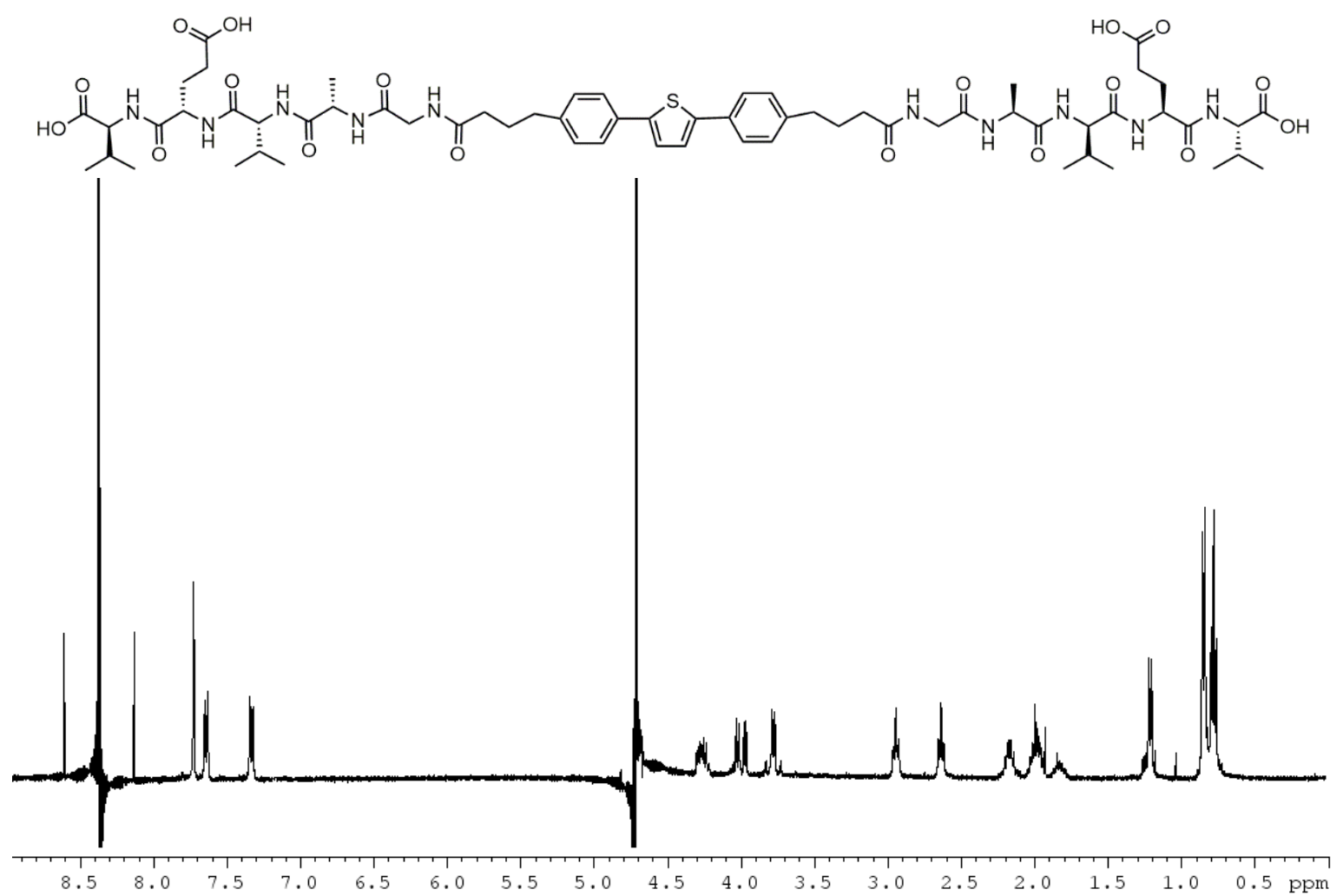

Figure S37. ${ }^{1} \mathrm{H}$ NMR $\left(\mathrm{D}_{2} \mathrm{O}, 400 \mathrm{MHz}\right)$ of $\mathbf{V}_{\mathbf{D}} \mathbf{A L}_{\mathbf{L}}-3$.
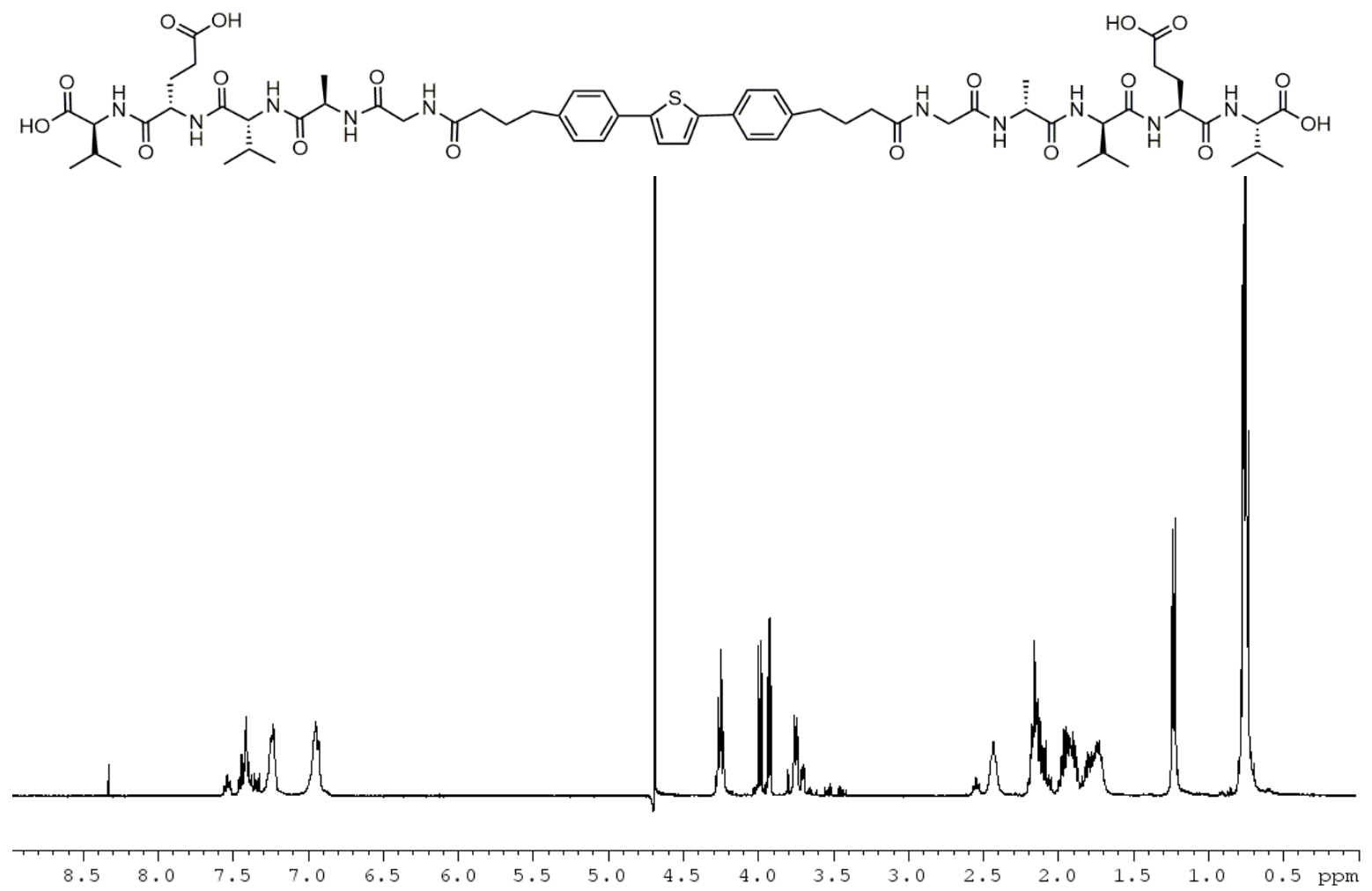

Figure S38. ${ }^{1} \mathrm{H}$ NMR $\left(\mathrm{D}_{2} \mathrm{O}, 400 \mathrm{MHz}\right)$ of $\mathbf{V}_{\mathbf{D}} \mathbf{A D}_{\mathbf{D}}-3$. 


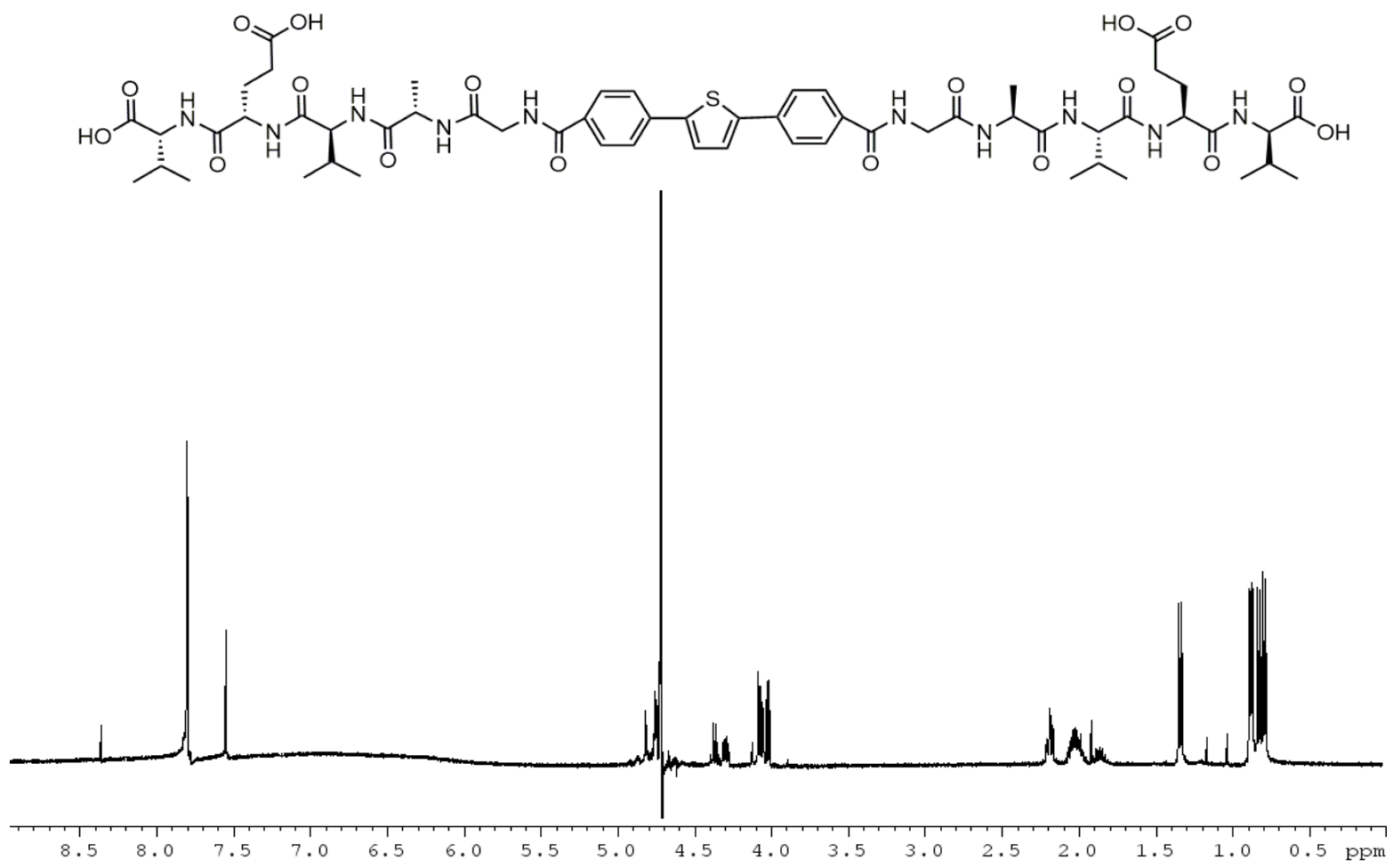

Figure S39. ${ }^{1} \mathrm{H}$ NMR $\left(\mathrm{D}_{2} \mathrm{O}, 400 \mathrm{MHz}\right)$ of $\mathbf{V}_{\mathbf{D}} \mathbf{E V}_{\mathbf{L}} \mathbf{A}_{\mathbf{L}}$ G-P-T-P-GAL $\mathbf{V}_{\mathbf{L}} \mathbf{E V}$. 


\section{Electron Microscopy \& TEM Images}

1D nanostructures were prepared through acidification of aqueous peptide stock solutions. The switching of valine and/or alanine configuration did not have any predictable impact on the supramolecular nanostructures formed from these peptide-spacer- $\pi$ molecules, although they did lead to significant and reproducible differences in terms of nanostructure width. Each of the molecules was observed to form 1D tape-like nanostructures but with different widths and persistence lengths: all the 0-spacer peptides yielded nanostructures over micrometers in length whereas 1-, 2- and 3-spacer peptides yielded nanostructures of only few hundred nanometers in length. $V_{L} A_{L}-0$ and $V_{D} A_{D}-0$ showed nanostructures of $11.5 \pm 0.5 \mathrm{~nm}$ and $12.4 \pm 0.6 \mathrm{~nm}$ in diameter whereas $V_{L} A_{D^{-}} 0$ showed relatively thicker nanostructures of widths around $18.3 \pm 0.7 \mathrm{~nm}$ and $\mathrm{V}_{\mathrm{D}} \mathrm{A}_{\mathrm{L}}-0$ showed nanostructures of widths $9.4 \pm 0.6 \mathrm{~nm}$ (Figure $\mathbf{S 4 0}$ ). Although this trend shows that subtle variation of the amino acid configuration impacts the global supramolecular structures formed, it is still somewhat anecdotal: in the one-carbon spacer library, $V_{L} A_{L}-1$ formed nanostructures of widths around $7.4 \pm 0.4 \mathrm{~nm}$ whereas $\mathrm{V}_{\mathrm{D}} \mathrm{A}_{\mathrm{L}}-1$ formed nanostructures of $10.3 \pm$ $0.6 \mathrm{~nm}$ width. Similarly, nanostructures of $\mathrm{V}_{L} A_{D^{-}} 1$ and $V_{D} A_{D^{-}}-1$ had widths of $9.5 \pm 0.3 \mathrm{~nm}$ and $10.2 \pm 0.4$ nm, respectively (Figure S41, Table S1).

This library of peptides uniformly afforded 1D tape-like nanostructures, thus providing a morphological normalization of the self-assembly process regardless of the initial molecular structure. Therefore, we are able to draw more direct structure-electronic function correlations in comparable nanomaterial structures without needing to consider simultaneous morphological variations. We could not observe any differences in finer superstructure details (e.g. coiling or twisting) within these nanostructures that could be correlated to the configuration of the molecular counterparts or supramolecular assemblies. 


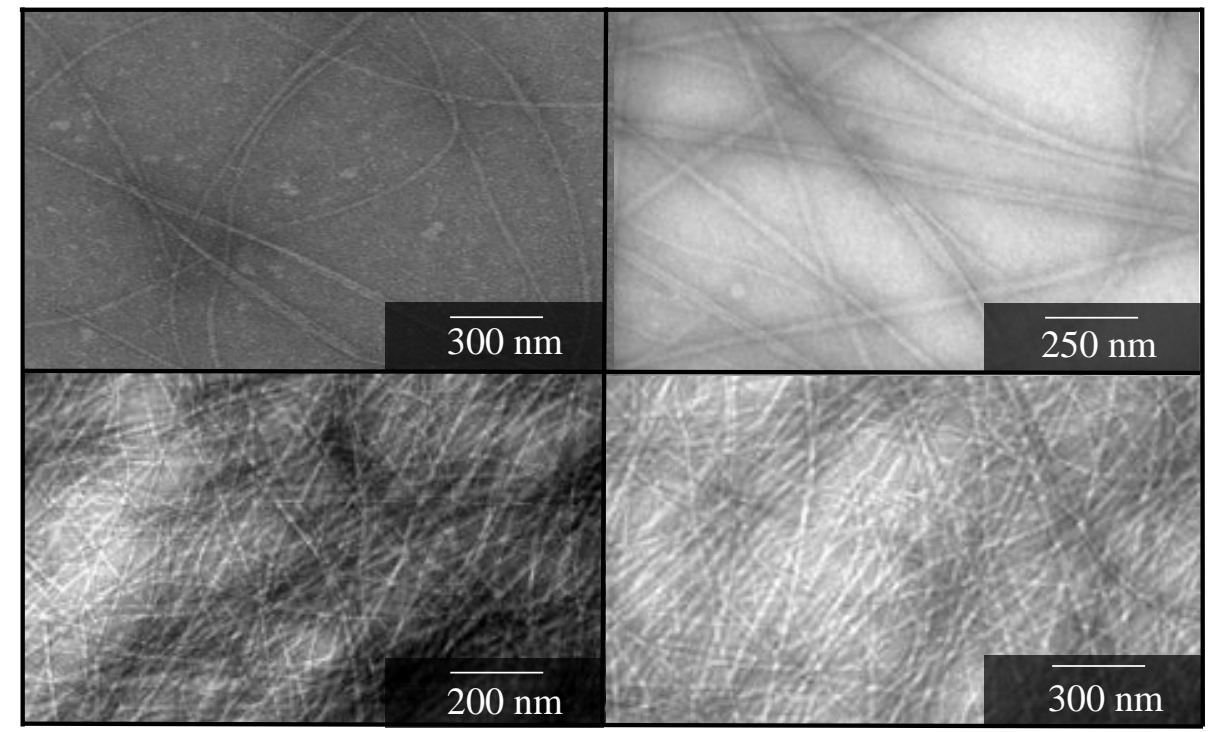

Figure S40. TEM images of peptide $\mathbf{V}_{\mathbf{L}} \mathbf{A}_{\mathbf{L}} \mathbf{- 0}$ (1 st row, left; $11.5 \pm 0.5 \mathrm{~nm}$ ), $\mathbf{V}_{\mathbf{L}} \mathbf{A}_{\mathbf{D}} \mathbf{- 0}$ (1 1 st row, right; $18.3 \pm 0.7 \mathrm{~nm}$ ), VDAL-0 (2nd row, right; $9.4 \pm 0.6 \mathrm{~nm}$ ), VDAd-0 (2nd row, left; $12.4 \pm 0.6 \mathrm{~nm}$ ). Nanostructure widths are mentioned in parentheses.

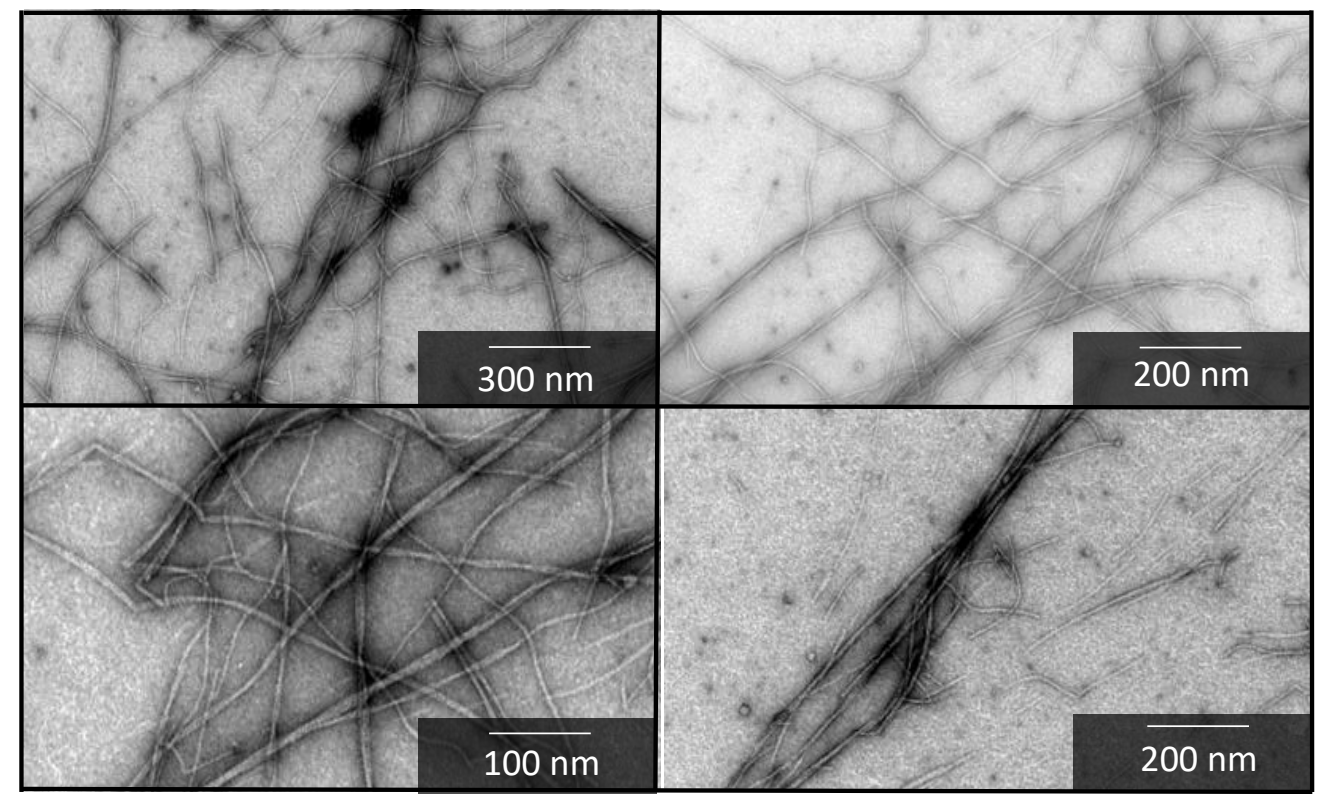

Figure S41. TEM images of peptide $\mathbf{V}_{\mathbf{L}} \mathbf{A L}_{\mathbf{L}} \mathbf{1}$ (1 1 st row, left; $\left.7.4 \pm 0.4 \mathrm{~nm}\right), \mathbf{V}_{\mathbf{L A d}} \mathbf{A} \mathbf{1}$ (1st row, right; $9.5 \pm 0.3 \mathrm{~nm}$ ), $\mathbf{V}_{\mathbf{D}} \mathbf{A}_{\mathbf{L}} \mathbf{- 1}$ (2nd row, right; $\left.10.3 \pm 0.6 \mathrm{~nm}\right), \mathbf{V}_{\mathbf{D}} \mathbf{A}_{\mathbf{D}}-1$ ( $2 \mathrm{nd}$ row, left; $10.2 \pm 0.4 \mathrm{~nm}$ ). Nanostructure widths are mentioned in parentheses. 


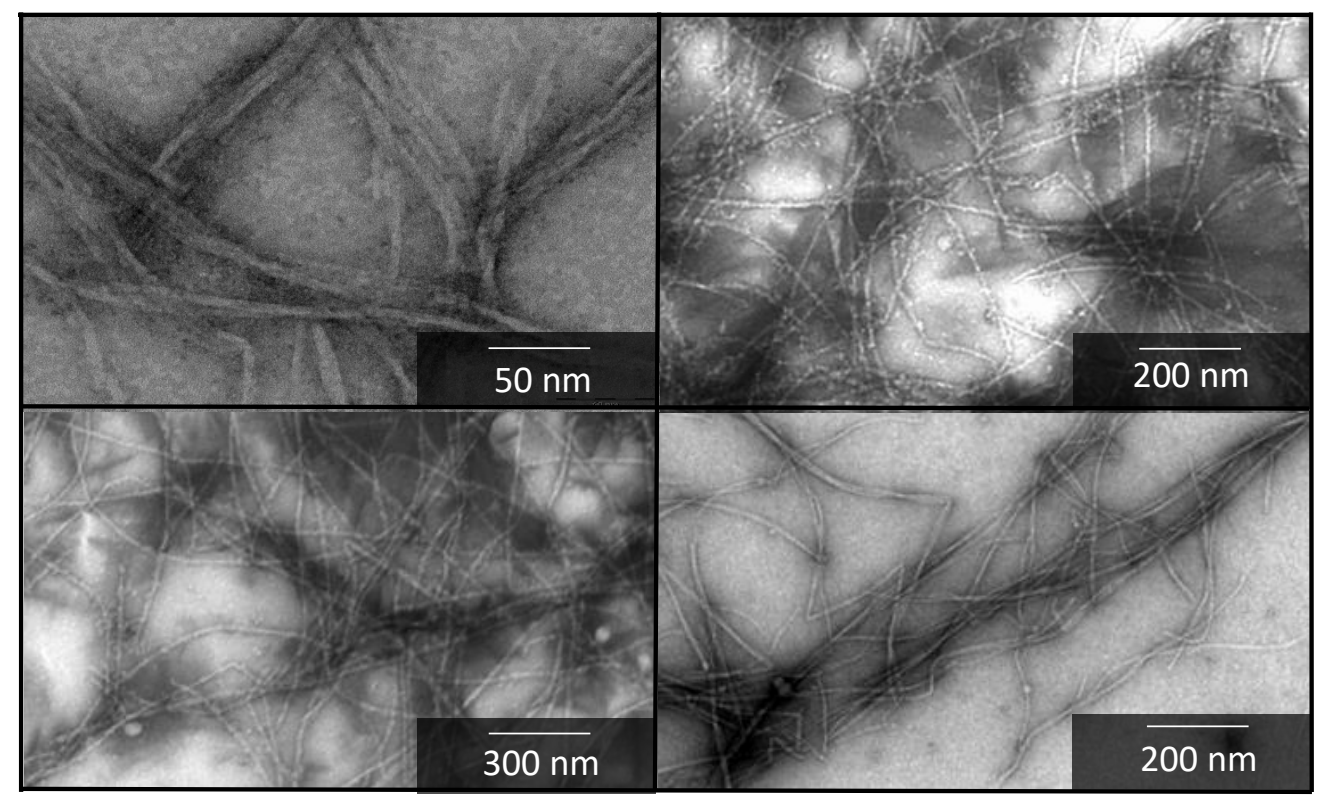

Figure S42. TEM images of peptide $\mathbf{V}_{\mathbf{L}} \mathbf{A}_{\mathbf{L}}-2$ (1st row, left; $6.1 \pm 0.8 \mathrm{~nm}$ ), $\mathbf{V}_{\mathbf{L}} \mathbf{A}_{\mathbf{D}}-2$ (1st row, right; $8.9 \pm 0.9 \mathrm{~nm}$ ), $\mathbf{V}_{\mathbf{D}} \mathbf{A}_{\mathbf{L}}-\mathbf{2}$ (2nd row, right; $\left.10.5 \pm 0.6 \mathrm{~nm}\right), \mathbf{V}_{\mathbf{D}} \mathbf{A}_{\mathbf{D}}-\mathbf{2}$ (2nd row, left; $9.7 \pm 0.8 \mathrm{~nm}$ ). Nanostructure widths are mentioned in parentheses.

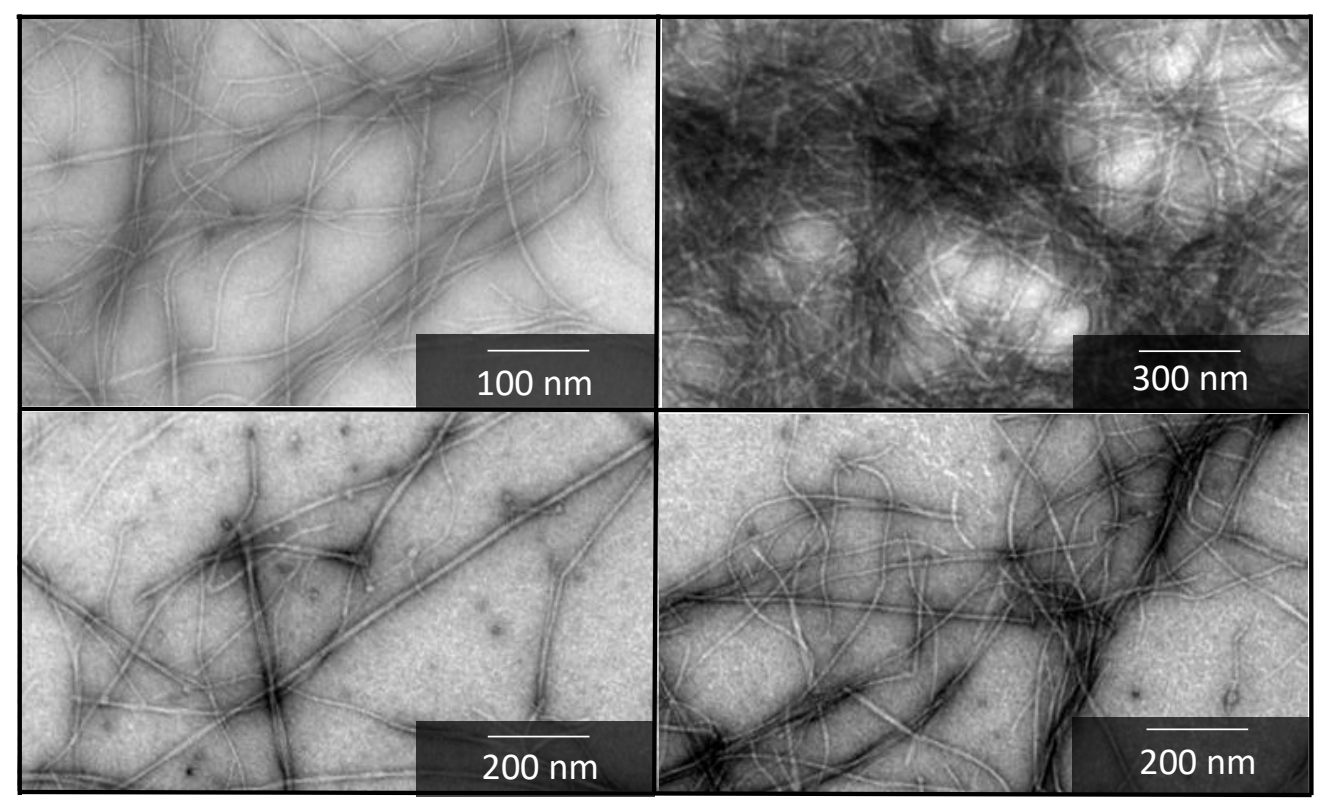

Figure S43. TEM images of peptide $\mathbf{V}_{\mathbf{L}} \mathbf{A}_{\mathbf{L}} \mathbf{- 3}$ (1 1 st row, left; $6.4 \pm 0.7 \mathrm{~nm}$ ), $\mathbf{V}_{\mathbf{L}} \mathbf{A}_{\mathbf{D}} \mathbf{3}$ (1st row, right; $6.3 \pm 0.6 \mathrm{~nm}$ ), $\mathbf{V}_{\mathbf{D}} \mathbf{A}_{\mathbf{L}} \mathbf{- 3}$ (2nd row, right; $\left.11.8 \pm 0.9 \mathrm{~nm}\right), \mathbf{V}_{\mathbf{D}} \mathbf{A}_{\mathbf{D}} \mathbf{- 3}$ (2nd row, left; $10.1 \pm 0.8 \mathrm{~nm}$ ). Nanostructure widths are mentioned in parentheses. 
Figure S44. TEM image of $\mathbf{V}_{\mathbf{D}} \mathbf{E V}_{\mathbf{L}} \mathbf{A}_{\mathbf{L}}$ G-P-T-P-GA $\mathbf{A}_{\mathbf{L}} \mathbf{V}_{\mathbf{L}} \mathbf{E V} \mathbf{D}(8.7 \pm 0.7 \mathrm{~nm})$. Nanostructure widths are mentioned in parentheses.

\begin{tabular}{|c|c|}
\hline & $\begin{array}{c}\text { Nanostructure } \\
\text { widths (nm) }\end{array}$ \\
\hline $\mathbf{V}_{\mathbf{L}} \mathbf{A}_{\mathbf{L}-\mathbf{0}}$ & $11.5 \pm 0.5$ \\
\hline$V_{L} A_{D}-0$ & $18.3 \pm 0.7$ \\
\hline $\mathbf{V}_{D} A_{L-0}$ & $9.4 \pm 0.6$ \\
\hline$V_{D A} A_{D}-0$ & $12.4 \pm 0.6$ \\
\hline$V_{L} A_{L-1}$ & $7.4 \pm 0.4$ \\
\hline$V_{L A D}-1$ & $9.5 \pm 0.3$ \\
\hline $\mathbf{V}_{D} A_{L-1}$ & $10.3 \pm 0.6$ \\
\hline$V_{D A D}-1$ & $10.2 \pm 0.4$ \\
\hline$V_{L} A_{L}-2$ & $6.1 \pm 0.8$ \\
\hline$V_{L} A_{D}-2$ & $8.9 \pm 0.9$ \\
\hline$V_{D} A_{L}-2$ & $10.5 \pm 0.6$ \\
\hline$V_{D A D}-2$ & $9.7 \pm 0.8$ \\
\hline$V_{L} A_{L-3}$ & $6.4 \pm 0.7$ \\
\hline$V_{L A D-3}$ & $6.3 \pm 0.6$ \\
\hline$V_{D} A_{L}-3$ & $11.8 \pm 0.9$ \\
\hline$V_{D A} A_{D-3}$ & $10.1 \pm 0.8$ \\
\hline $\begin{array}{c}V_{D E} E V_{L} A_{L} G-P-T-P- \\
G A_{L} V_{L} E V_{D}\end{array}$ & $8.7 \pm 0.7$ \\
\hline
\end{tabular}

Table S1. Nanostructure widths of different peptides as observed in TEM. 


\section{DLS Spectra}
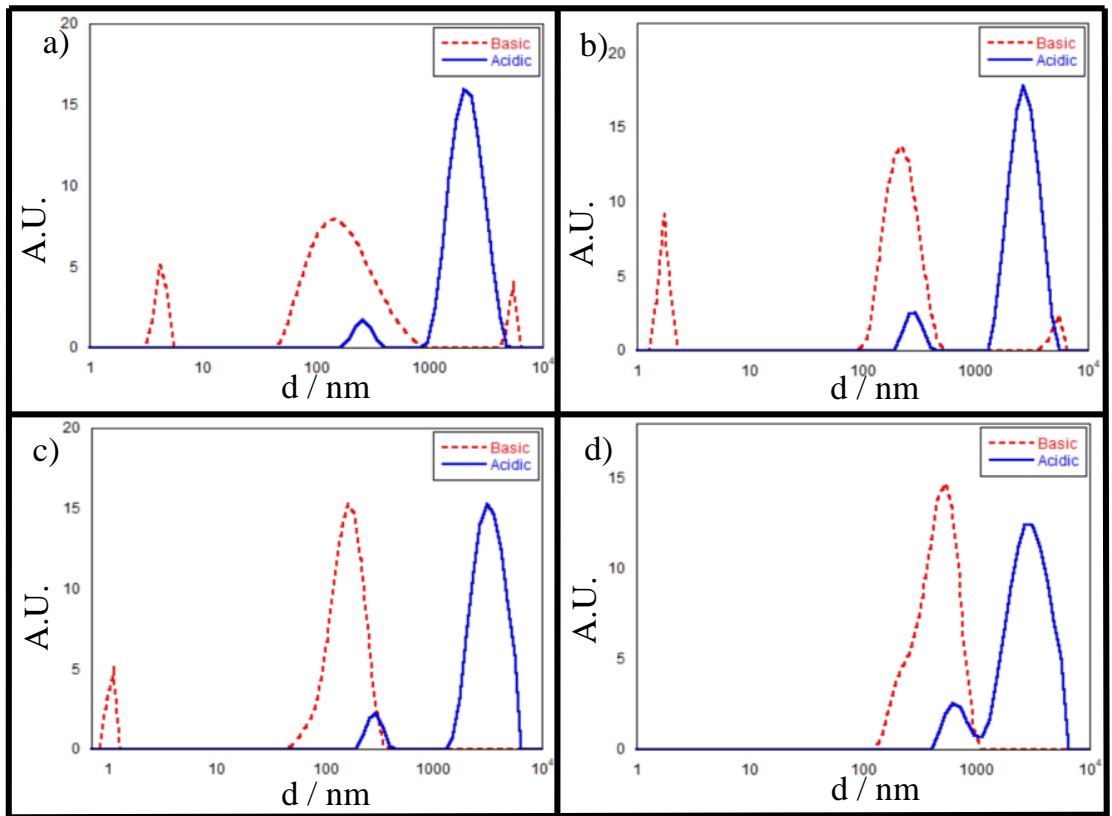

Figure $\mathbf{S 4 5}$. Dynamic Light Scattering Spectra of peptide $\mathbf{V}_{\mathbf{L}} \mathbf{A}_{\mathbf{L}} \mathbf{- 0}$ (1 st row, left), $\mathbf{V}_{\mathbf{L}} \mathbf{A}_{\mathbf{D}} \mathbf{- 0}$ (1st row, right), $\mathbf{V}_{\mathbf{D}} \mathbf{A}_{\mathbf{L}}-\mathbf{0}$ (2nd row, right), $\mathbf{V}_{\mathbf{D}} \mathbf{A}_{\mathbf{D}}-\mathbf{0}$ (2nd row, left)
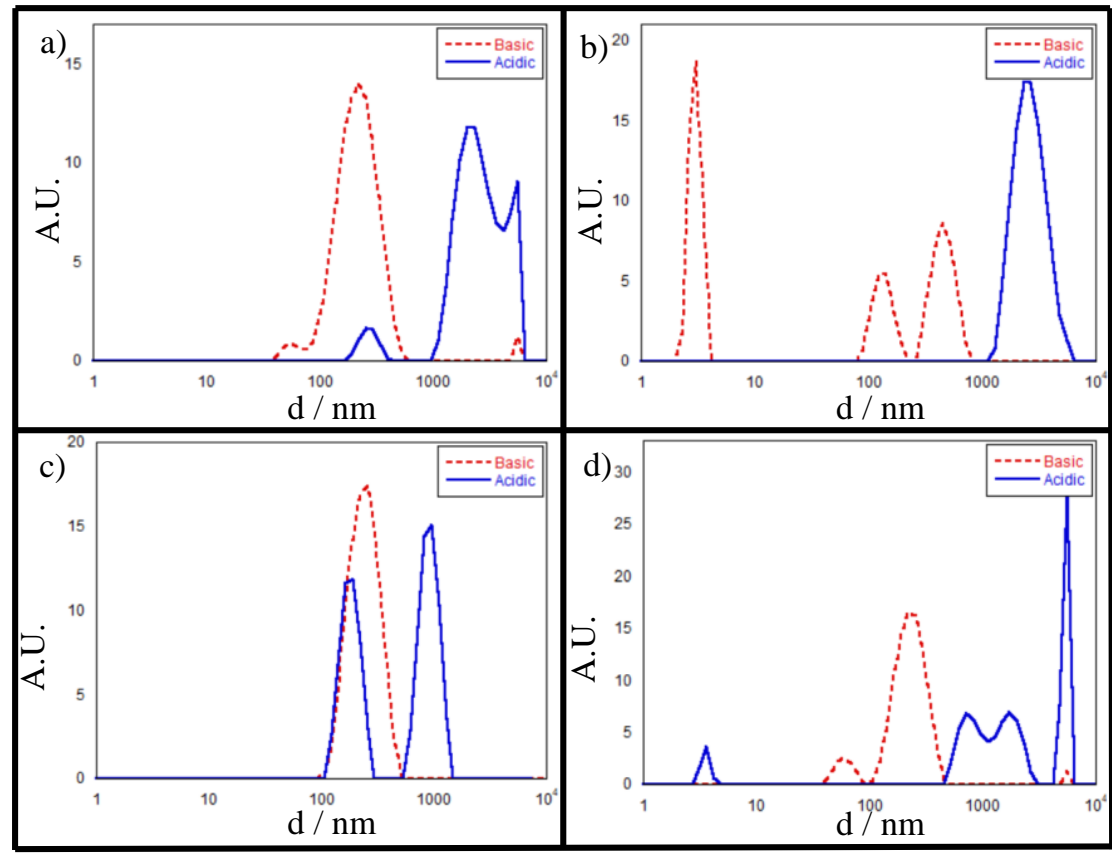

Figure S46. Dynamic Light Scattering spectra of peptide $\mathbf{V}_{\mathbf{L}} \mathbf{A}_{\mathbf{L}} \mathbf{- 1}$ (1 st row, left), $\mathbf{V}_{\mathbf{L}} \mathbf{A}_{\mathbf{D}}-1$ (1 st row, right), $\mathbf{V}_{\mathbf{D}} \mathbf{A}_{\mathbf{L}} \mathbf{- 1}$ (2nd row, right), $\mathbf{V}_{\mathbf{D}} \mathbf{A}_{\mathbf{D}} \mathbf{- 1}$ (2nd row, left) 


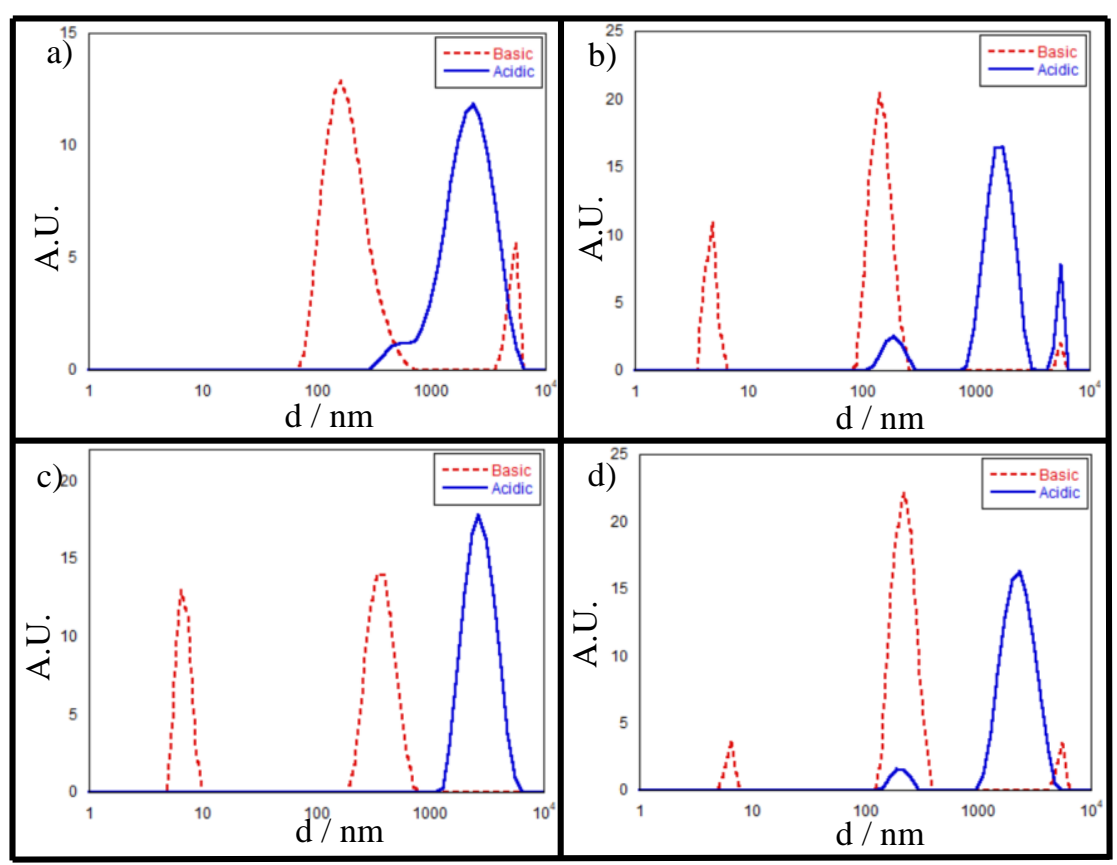

Figure S47. Dynamic Light Scattering spectra of peptide $\mathbf{V}_{\mathbf{L}} \mathbf{A}_{\mathbf{L}}-\mathbf{2}$ (1st row, left), $\mathbf{V}_{\mathbf{L}} \mathbf{A}_{\mathbf{D}}-\mathbf{2}$ (1st row, right), $\mathbf{V}_{\mathbf{D}} \mathbf{A}_{\mathbf{L}}-\mathbf{2}$ (2nd row, right), $\mathbf{V}_{\mathbf{D}} \mathbf{A}_{\mathbf{D}}-\mathbf{2}$ (2nd row, left)
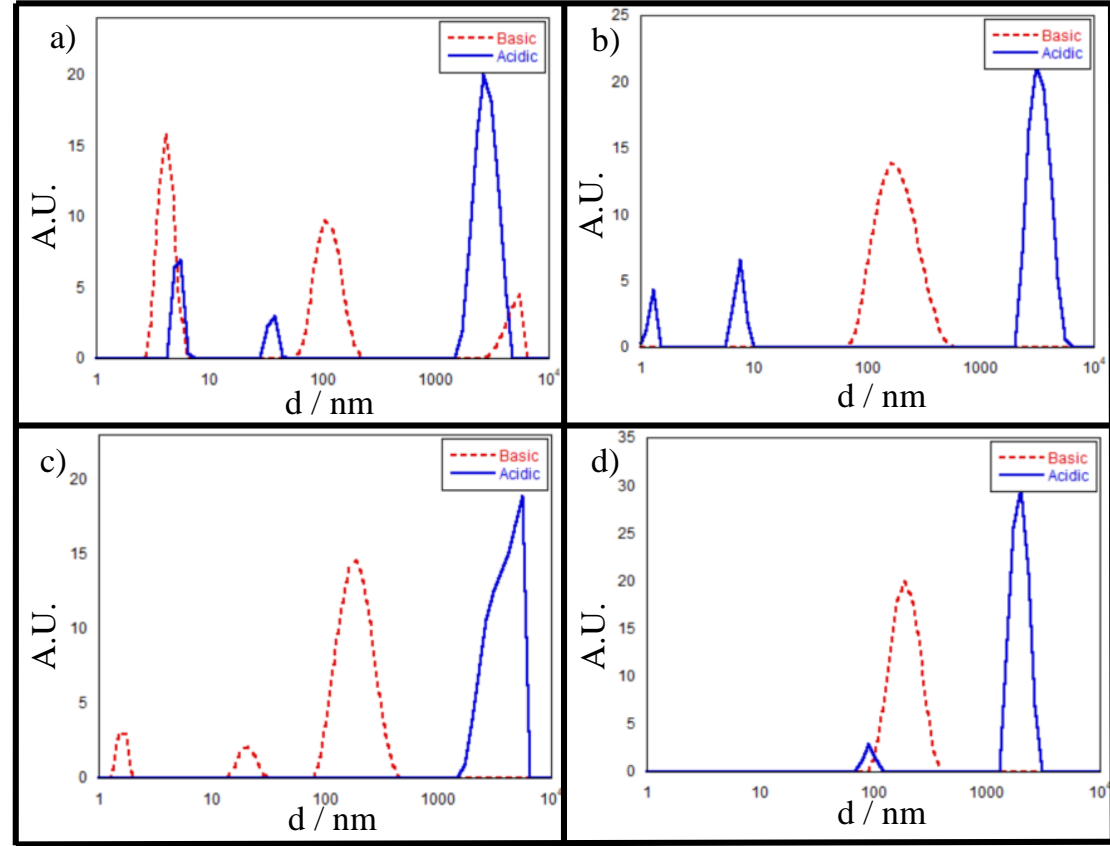

Figure S48. Dynamic Light Scattering spectra of peptide $V_{\mathbf{L A}} \mathbf{A}-3$ (1st row, left), VLAd-3 (1st row, right), $\mathbf{V}_{\mathbf{D}} \mathbf{A}_{\mathbf{L}}-\mathbf{3}$ (2nd row, right), $\mathbf{V}_{\mathbf{D}} \mathbf{A}_{\mathbf{D}} \mathbf{- 3}$ (2nd row, left) 


\section{FT-IR Spectra}

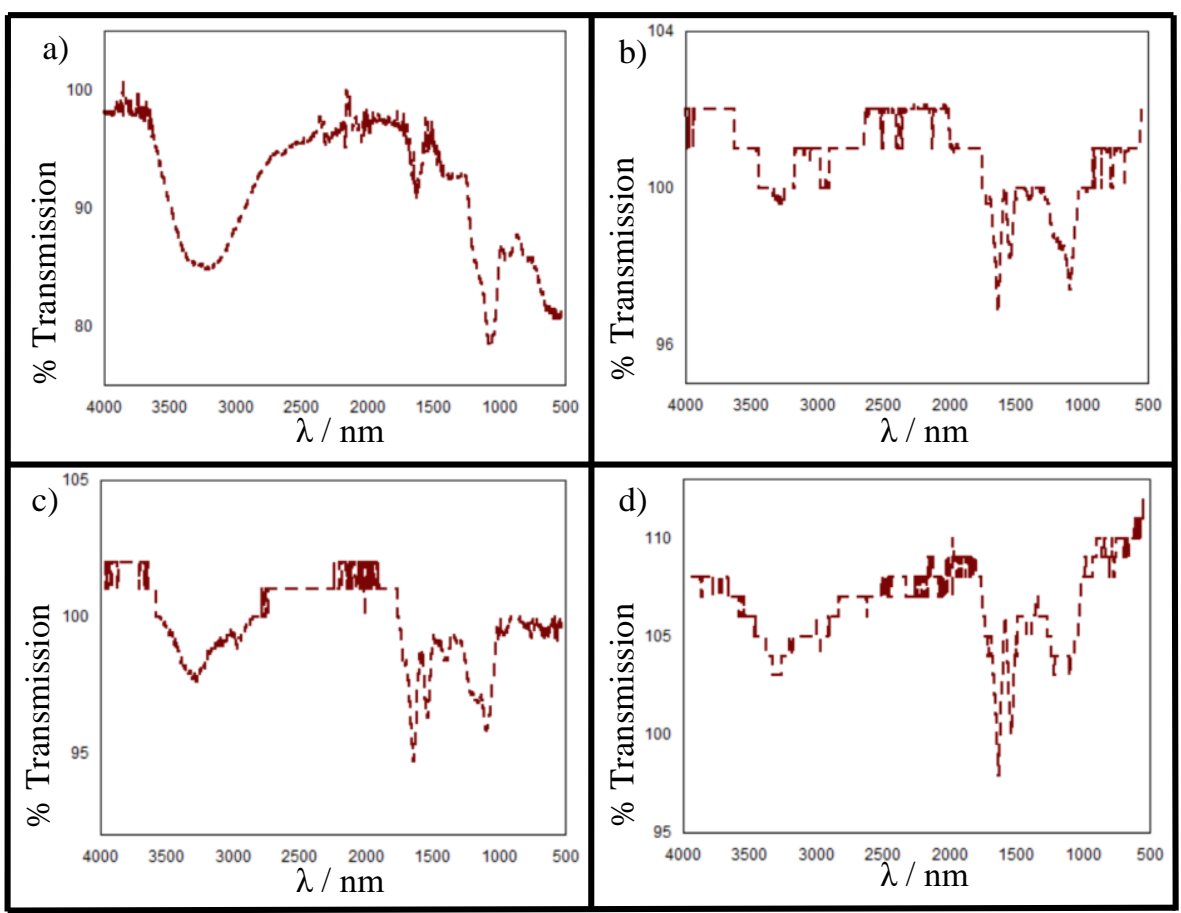

Figure S49. FT-IR spectra of peptide $\mathbf{V}_{\mathbf{L}} \mathbf{A L}_{\mathbf{L}-0}$ (1st row, left), $\mathbf{V}_{\mathbf{L A D}} \mathbf{A D}_{\mathbf{0}}$ (1st row, right), $\mathbf{V}_{\mathbf{D}} \mathbf{A L}_{\mathbf{L}} \mathbf{0}$ (2nd row, right), $\mathbf{V}_{\mathbf{D}} \mathbf{A}_{\mathbf{D}} \mathbf{- 0}$ (2nd row, left)

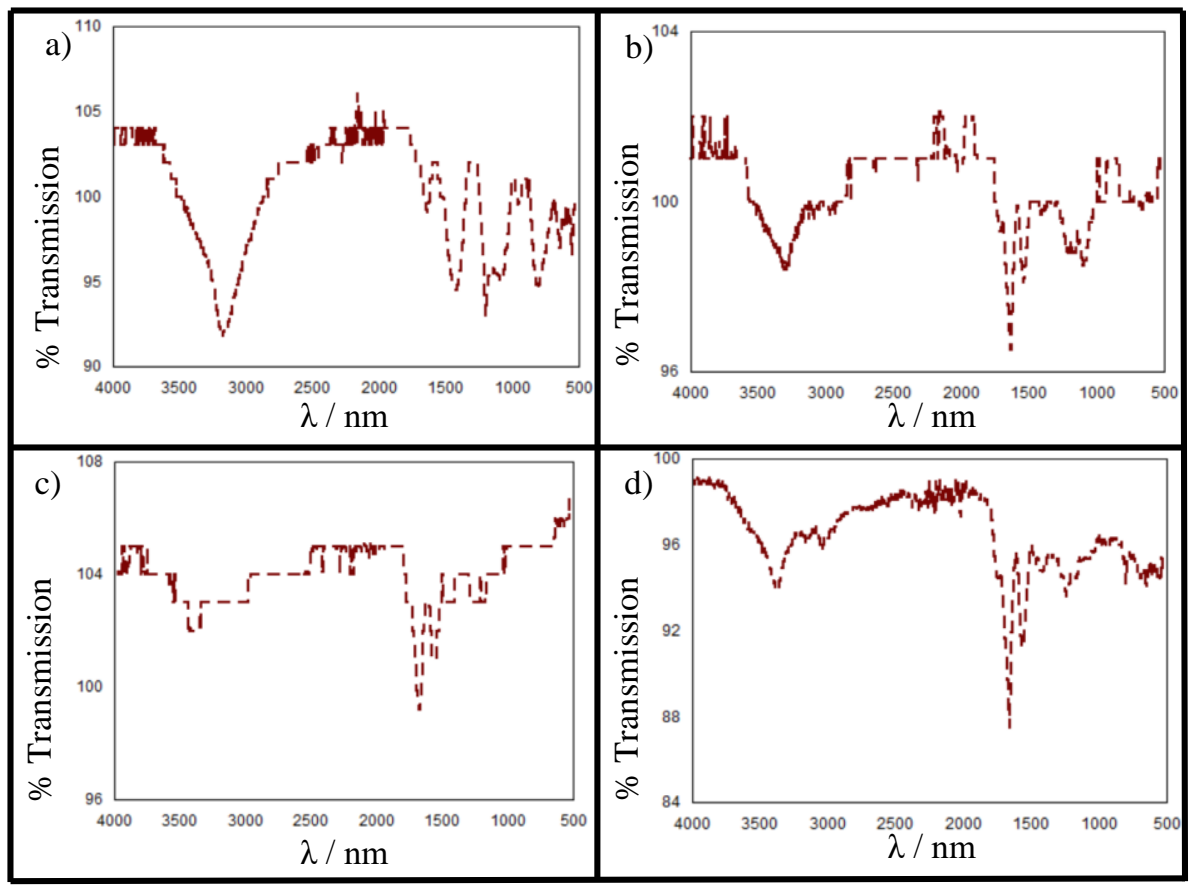

Figure S50. FT-IR spectra of peptide $\mathbf{V}_{\mathbf{L}} \mathbf{A}_{\mathbf{L}} \mathbf{- 1}$ (1st row, left), VLAD-1 (1st row, right), VDAL-1 (2nd row, right), VDAD-1 (2nd row, left) 


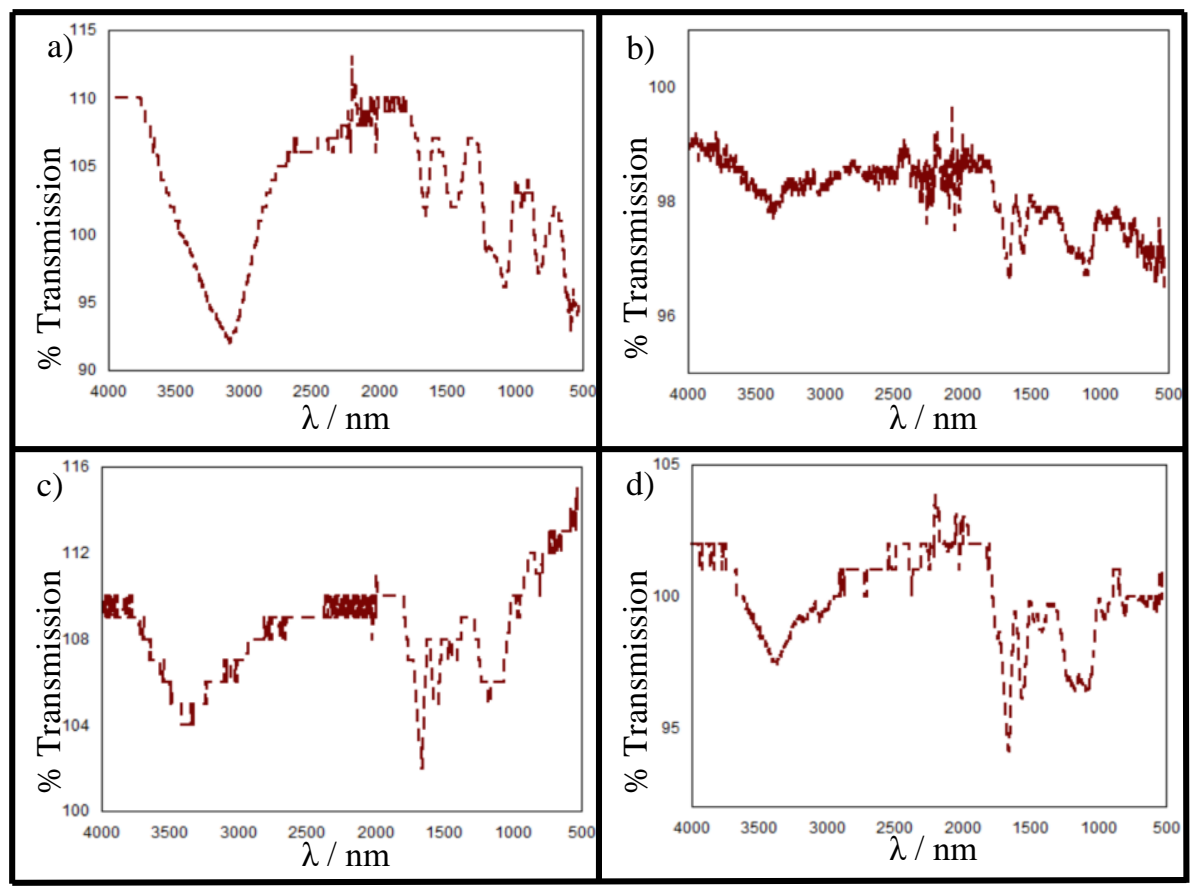

Figure S51. FT-IR spectra of peptide $\mathbf{V}_{\mathbf{L}} \mathbf{A}_{\mathbf{L}}-\mathbf{2}$ (1st row, left), $\mathbf{V}_{\mathbf{L}} \mathbf{A}_{\mathbf{D}} \mathbf{- 2}$ (1st row, right), $\mathbf{V}_{\mathbf{D}} \mathbf{A}_{\mathbf{L}} \mathbf{- 2}$ (2nd row, right), $V_{\mathbf{D}} \mathbf{A}_{\mathbf{D}}-\mathbf{2}$ (2nd row, left)

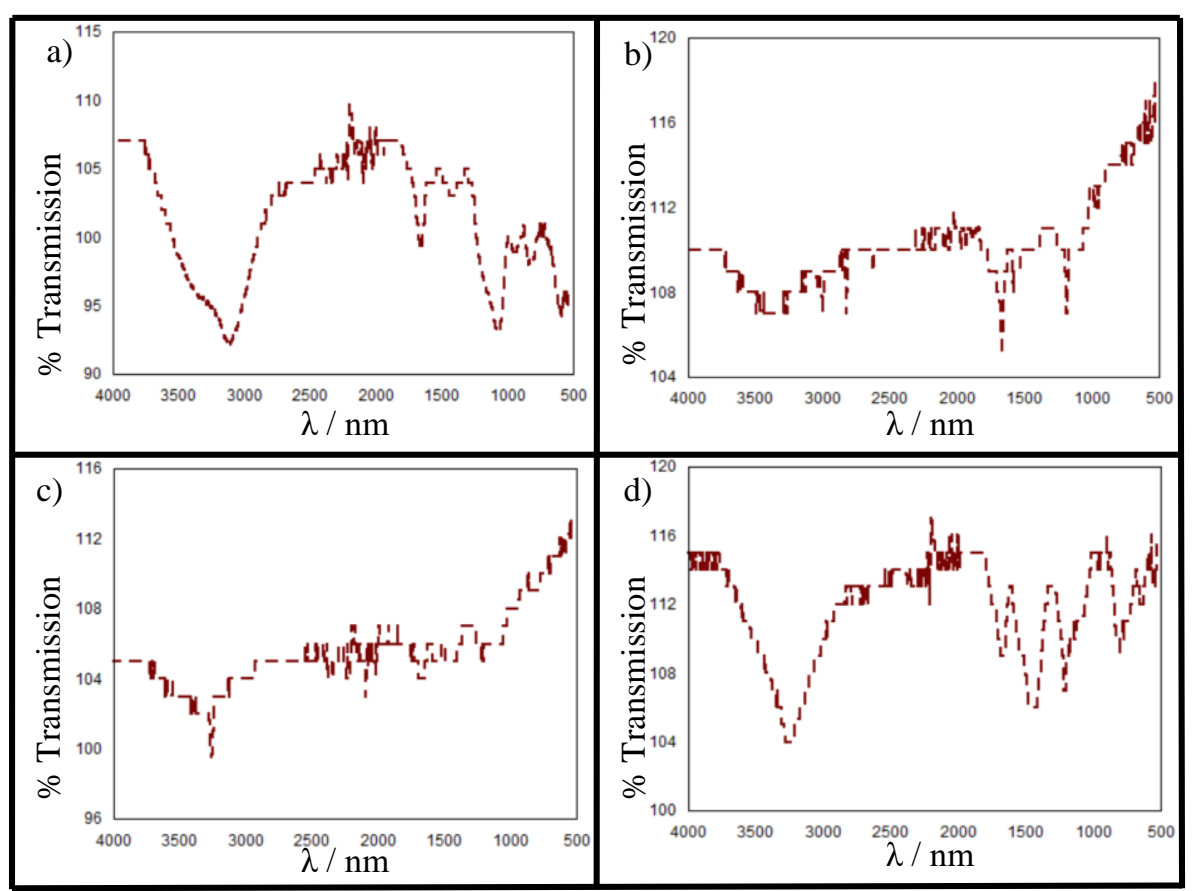

Figure S52. FT-IR spectra of peptide $\mathbf{V}_{\mathbf{L}} \mathbf{A}_{\mathbf{L}} \mathbf{- 3}$ (1st row, left), $\mathbf{V}_{\mathbf{L}} \mathbf{A}_{\mathbf{D}} \mathbf{- 3}$ (1st row, right), $\mathbf{V}_{\mathbf{D}} \mathbf{A}_{\mathbf{L}} \mathbf{- 3}$ (2nd row, right), $\mathbf{V}_{\mathbf{D}} \mathbf{A}_{\mathbf{D}-\mathbf{3}}$ (2nd row, left) 


\section{UV-Vis Spectra}

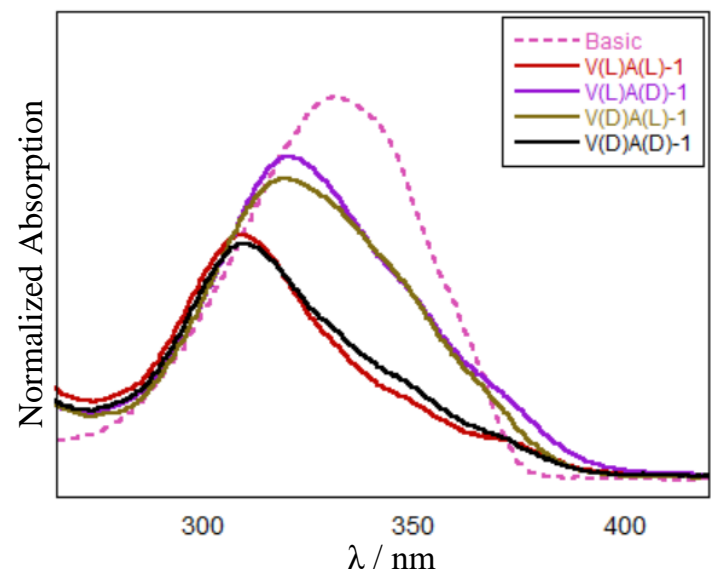

Figure S53. UV-Vis spectra of 1-spacer peptides in basic and acidic solution acquired at a concentration of $20 \mu \mathrm{M}$.

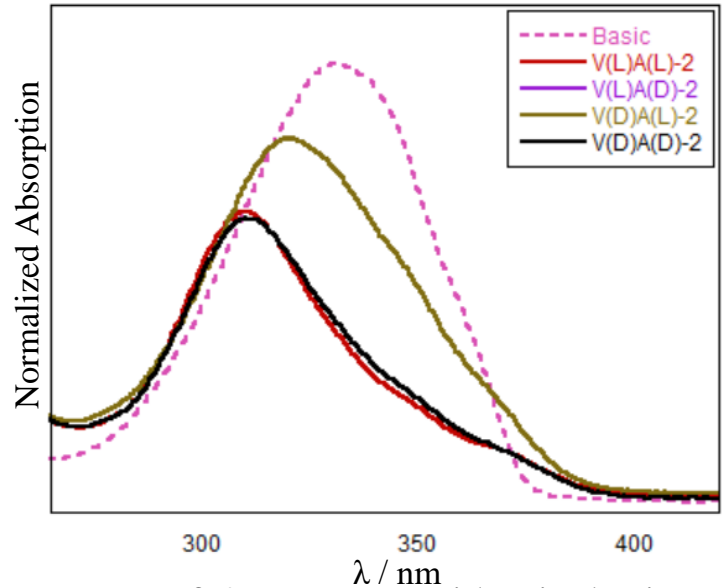

Figure S54. UV-Vis spectra of 2-spacer peptides in basic and acidic solution acquired at a concentration of $20 \mu \mathrm{M}$.

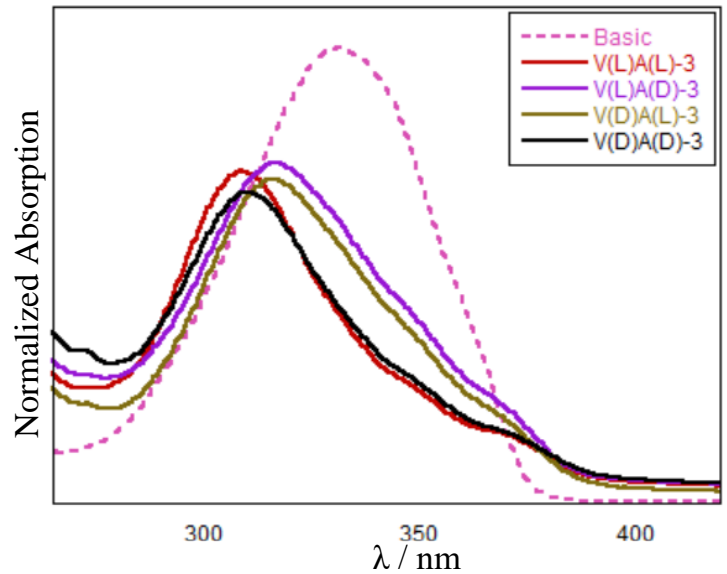

Figure S55. UV-Vis spectra of 3-spacer peptides in basic and acidic solution acquired at a concentration of $20 \mu \mathrm{M}$. 


\begin{tabular}{|c|c|c|c|}
\hline & Basic $(\mathrm{nm})$ & Acidic $(\mathrm{nm})$ & Shift $(\mathrm{nm})$ \\
\hline $\mathbf{V}_{\mathbf{L}} \mathbf{A}_{\mathbf{L}}-\mathbf{0}$ & 351 & 322 & 29 \\
\hline $\mathbf{V}_{\mathbf{L}} \mathbf{A}_{\mathbf{D}}-\mathbf{0}$ & 350 & 344 & 6 \\
\hline $\mathbf{V}_{\mathbf{D}} \mathbf{A}_{\mathbf{L}}-\mathbf{0}$ & 350 & 348 & 2 \\
\hline $\mathbf{V}_{\mathbf{D}} \mathbf{A}_{\mathbf{D}}-\mathbf{0}$ & 352 & 322 & 30 \\
\hline $\mathbf{V}_{\mathbf{L}} \mathbf{A}_{\mathbf{L}}-\mathbf{1}$ & 332 & 309 & 23 \\
\hline $\mathbf{V}_{\mathbf{L}} \mathbf{A}_{\mathbf{D}}-\mathbf{1}$ & 331 & 322 & 9 \\
\hline $\mathbf{V}_{\mathbf{D}} \mathbf{A}_{\mathbf{L}}-\mathbf{1}$ & 331 & 321 & 10 \\
\hline $\mathbf{V}_{\mathbf{D}} \mathbf{A}_{\mathbf{D}}-\mathbf{1}$ & 332 & 310 & 22 \\
\hline $\mathbf{V}_{\mathbf{L}} \mathbf{A}_{\mathbf{L}}-\mathbf{2}$ & 331 & 309 & 22 \\
\hline $\mathbf{V}_{\mathbf{L}} \mathbf{A}_{\mathbf{D}}-\mathbf{2}$ & 330 & 317 & 13 \\
\hline $\mathbf{V}_{\mathbf{D}} \mathbf{A}_{\mathbf{L}}-\mathbf{2}$ & 331 & 317 & 14 \\
\hline $\mathbf{V}_{\mathbf{D}} \mathbf{A}_{\mathbf{D}}-\mathbf{2}$ & 331 & 309 & 22 \\
\hline $\mathbf{V}_{\mathbf{L}} \mathbf{A}_{\mathbf{L}}-\mathbf{3}$ & 332 & 308 & 24 \\
\hline $\mathbf{V}_{\mathbf{L}} \mathbf{A}_{\mathbf{D}}-\mathbf{3}$ & 330 & 315 & 15 \\
\hline $\mathbf{V}_{\mathbf{D}} \mathbf{A}_{\mathbf{L}}-\mathbf{3}$ & 331 & 316 & 15 \\
\hline $\mathbf{V}_{\mathbf{D}} \mathbf{A}_{\mathbf{D}}-\mathbf{3}$ & 332 & 310 & 22 \\
\hline $\mathbf{V}_{\mathbf{D E}} \mathbf{V}_{\mathbf{L}} \mathbf{A}_{\mathbf{L}} \mathbf{G}-\mathbf{P}-$ & 351 & 323 & 28 \\
T-P-GAAL & & & \\
\hline
\end{tabular}

Table S2. Absorption maxima of peptides at different condition (e.g. basic, acidic). 


\section{PL Spectra}

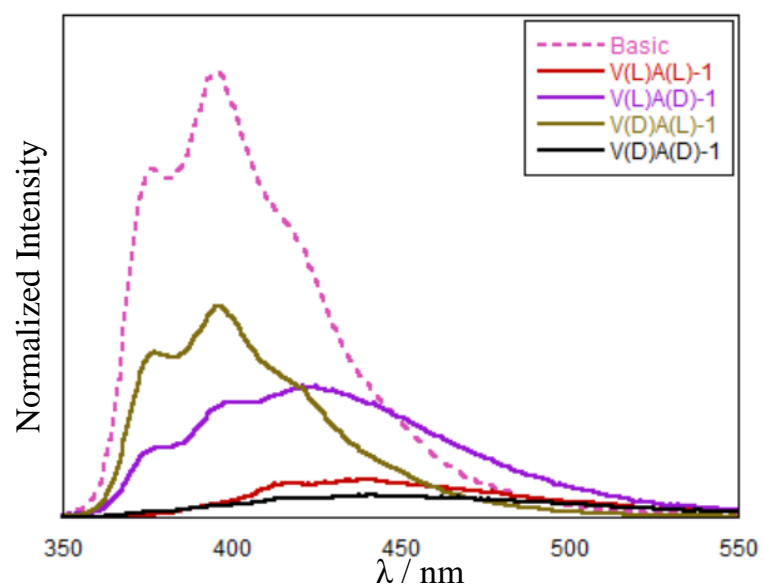

Figure S56. PL spectra of 1-spacer peptides in basic and acidic solution acquired at a concentration of $10 \mu \mathrm{M}$

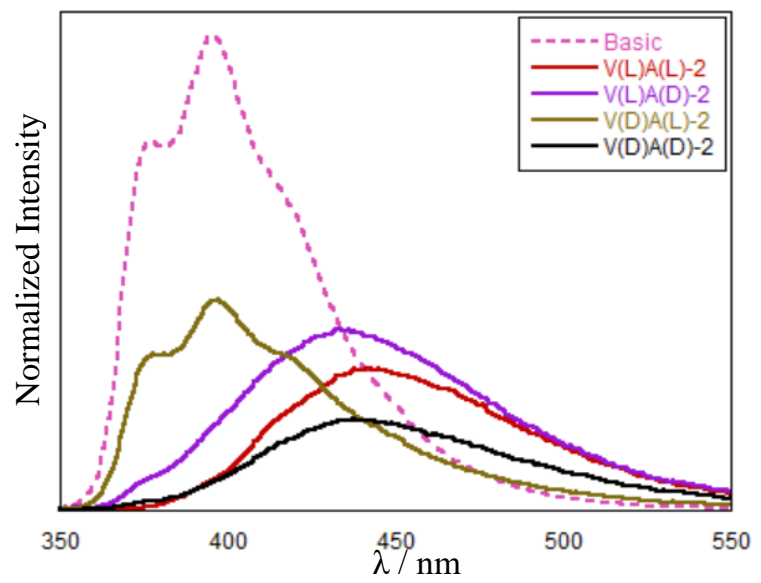

Figure S57. PL spectra of 2-spacer peptides in basic and acidic solution acquired at a concentration of $10 \mu \mathrm{M}$

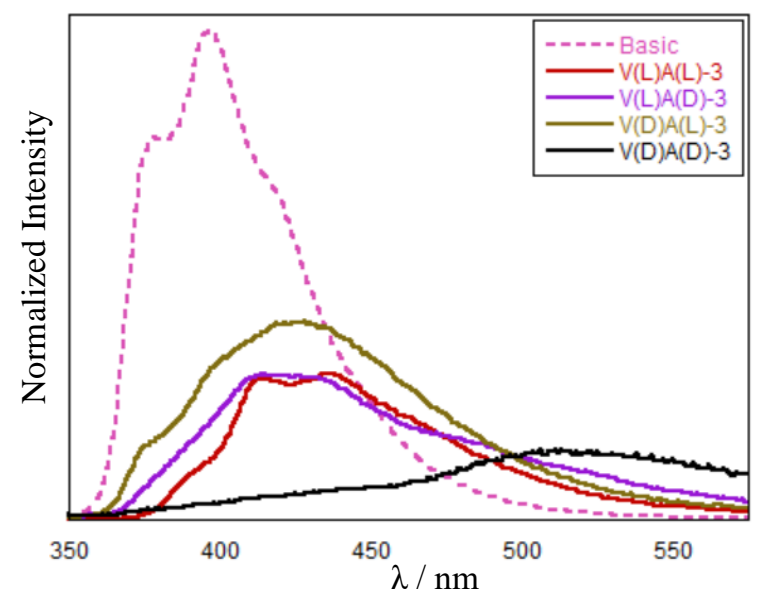

Figure S58. PL spectra of 3-spacer peptides in basic and acidic solution acquired at a concentration of $10 \mu \mathrm{M}$ 


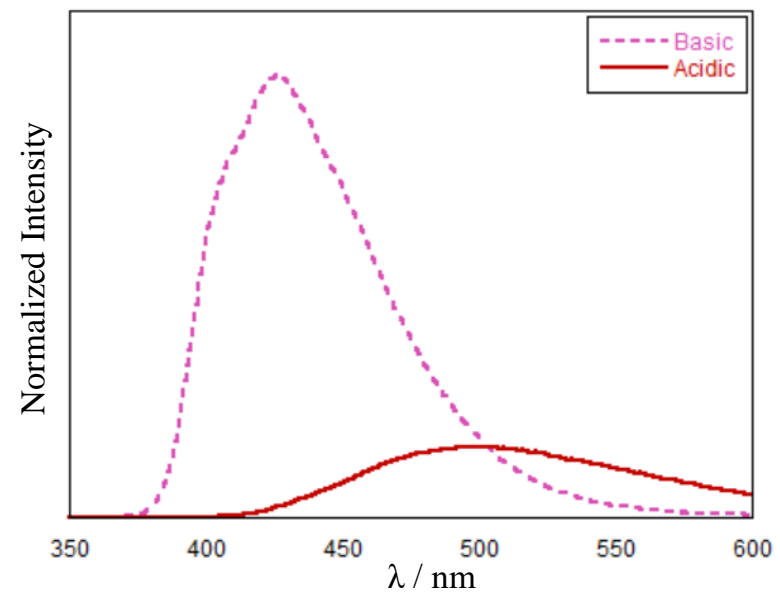

Figure S59. PL spectra of $V_{D E} V_{L} A_{L}$ G-P-T-P-GA $\mathbf{G}_{\mathbf{L}} \mathbf{V}_{\mathbf{L}} \mathbf{E V} V_{\mathbf{D}}$ peptide in basic and acidic solution acquired at a concentration of $10 \mu \mathrm{M}$ 


\section{Near-UV CD Spectra}
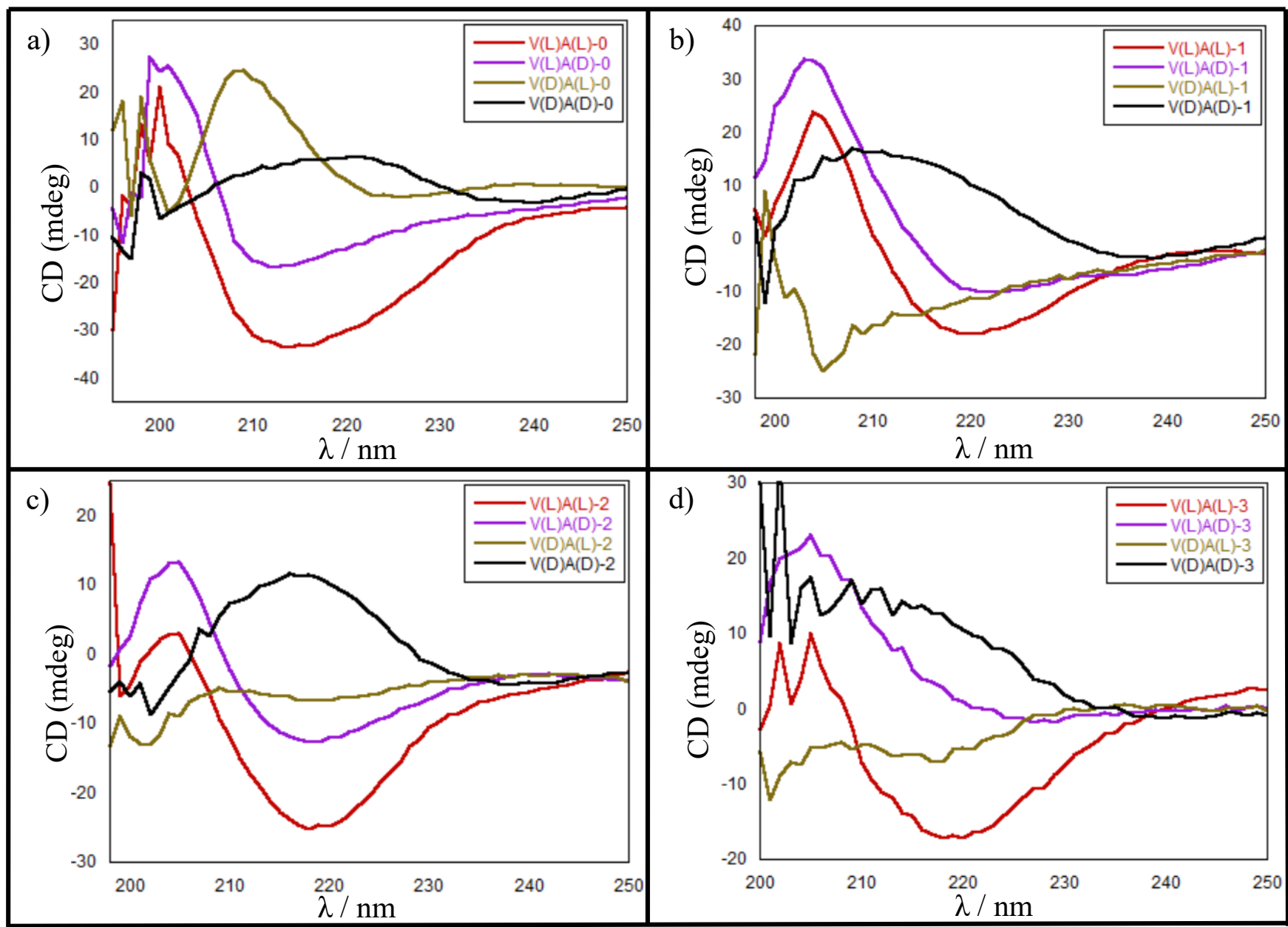

Figure S60. CD spectroscopy at near-UV region of (a) 0-spacer, (b) 1-spacer, (c) 2-spacer and (d) 3 -spacer under acidic condition at a concentration of $25 \mu \mathrm{M}$. Basic solutions were made by adding $30 \mu \mathrm{L}$ of $\mathrm{KOH}(2 \mathrm{M})$ and acidified by adding $50 \mu \mathrm{L}$ of $\mathrm{HCl}(2 \mathrm{M})$ to the basic solution. 


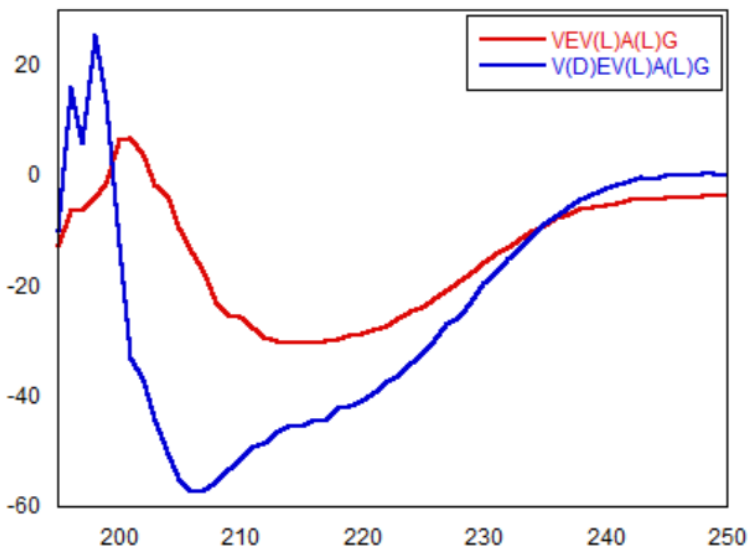

Figure S61. $C D$ spectroscopy at near-UV region of of $V_{L} A_{L}-0$ and $V_{D} E V_{L} A_{L} G-0$ under acidic condition at a concentration of $25 \mu \mathrm{M}$. Basic solutions were made by adding $30 \mu \mathrm{L}$ of $\mathrm{KOH}(2 \mathrm{M})$ and acidified by adding $50 \mu \mathrm{L}$ of $\mathrm{HCl}(2 \mathrm{M})$ to the basic solution.

\begin{tabular}{|c|c|c|c|}
\hline & $\begin{array}{c}\text { Experimental } \\
\text { Chirality of } \\
\text { Nanostructure }\end{array}$ & $\begin{array}{c}\text { Exciton } \\
\text { Coupling? }\end{array}$ & $\begin{array}{l}\text { Computational } \\
\text { Chirality of } \\
\text { Nanostructure }\end{array}$ \\
\hline$V_{L} A_{L-0}$ & M- strong & Yes & $\mathrm{M}$ \\
\hline$V_{L} A_{D-0}$ & M-weak & $\mathrm{No}$ & $\mathrm{P}$ \\
\hline $\mathbf{V}_{\mathrm{DA}} \mathbf{A}_{\mathrm{L}} \mathbf{0}$ & P-weak & $\mathrm{No}$ & $\mathrm{M}$ \\
\hline VDAD-0 & P-weak & Yes & $\mathrm{M}$ \\
\hline$V_{L} A_{L-1}$ & P-weak & Yes & $\mathrm{M}$ \\
\hline $\mathrm{V}_{L} A_{D-1}$ & M-weak & Yes & No Preference \\
\hline $\mathbf{V}_{D} A_{L-1}$ & M-strong & Yes & $\mathrm{M}$ \\
\hline $\mathbf{V}_{D} A_{D-1}$ & P-weak & Yes & $\mathrm{P}$ \\
\hline$V_{L} A_{L-2}$ & M-strong & Yes & $\mathrm{M}$ \\
\hline$V_{L A D-2}$ & M-weak & $\mathrm{No}$ & $\mathrm{M}$ \\
\hline$V_{D} A_{L-2}$ & M-weak & Yes & $\mathrm{M}$ \\
\hline $\mathrm{V}_{D A D}-2$ & P-strong & Yes & No preference \\
\hline$V_{L} A_{L-3}$ & P-weak & Yes & No preference \\
\hline $\mathrm{V}_{L} A_{D-3}$ & P-weak & Yes & $\mathrm{M}$ \\
\hline$V_{D A} A_{L}$ & M-strong & Yes & $\mathrm{M}$ \\
\hline $\mathrm{V}_{\mathrm{D}} \mathrm{A}_{\mathrm{D}}-\mathbf{3}$ & P-weak & Yes & $\mathrm{M}$ \\
\hline $\begin{array}{l}V_{D E V} E A_{L} G-P- \\
\text { T-P-GA } \\
\end{array}$ & M-strong & Yes & - \\
\hline
\end{tabular}

Table S3. Supramolecular chirality of nanostructures as observed in CD spectroscopy. 


\section{Metadynamics Convergence}
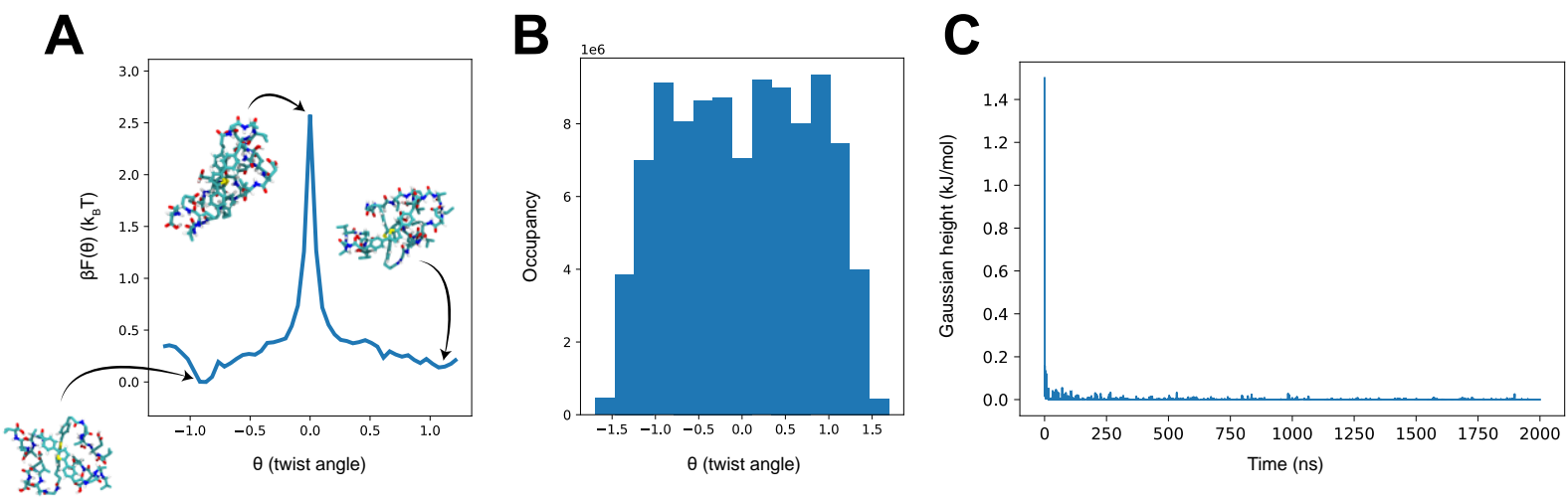

Figure S62. Metadynamics simulation and convergence criterion for an exemplar V(L)A(L) ethyl spacer system. (A) A potential of mean force (PMF) is estimated from the metadynamics simulation along the collective variable $\theta$ defining the signed twist angle between a dimerized pair of pi-conjugated peptides (Eqn. 1). Insets display simulation snapshots dimer at the indicated twist angles. (B) Histogram of the distribution of sampling along the signed twist angle CV $\theta$ induced by the converged metadynamics potential. We observe an approximately flat histogram in $\theta$ defined by the fraction of configurations with $\theta>0$ and $\theta<0$ lying within $\sim 5 \%$. (C) Time evolution of the height of the deposited Gaussians over the course of the metadynamics simulation. The plateau to zero is a second indicator of convergence of the metadynamics run such that the accumulated bias is no longer changing in time.

\section{Reference}

1. Panda, S. S.; Shmilovich, K.; Ferguson, A. L.; Tovar, J. D. Controlling Supramolecular Chirality in Peptide- $\pi$-Peptide Networks by Variation of the Alkyl Spacer Length. Langmuir 2019, 35, 14060-14073. 